Portland State University

PDXScholar

Fall 12-7-2017

\title{
Designing In-Headset Authoring Tools for Virtual Reality Video
}

Cuong Nguyen

Portland State University

Follow this and additional works at: https://pdxscholar.library.pdx.edu/open_access_etds

Part of the Computer Sciences Commons

Let us know how access to this document benefits you.

\section{Recommended Citation}

Nguyen, Cuong, "Designing In-Headset Authoring Tools for Virtual Reality Video" (2017). Dissertations and Theses. Paper 4037.

https://doi.org/10.15760/etd.5921

This Dissertation is brought to you for free and open access. It has been accepted for inclusion in Dissertations and Theses by an authorized administrator of PDXScholar. Please contact us if we can make this document more accessible: pdxscholar@pdx.edu. 
Designing In-Headset Authoring Tools for Virtual Reality Video

by

Cuong Nguyen

A dissertation submitted in partial fulfillment of the requirements for the degree of

\author{
Doctor of Philosophy \\ in \\ Computer Science
}
Dissertation Committee:
Feng Liu, Chair
Bart Massey
Wu-chi Feng
Miguel Andres Figliozzi

Portland State University 
(C) 2017 Cuong Nguyen 


\begin{abstract}
Virtual Reality (VR) video is emerging as a new art form. Viewing VR video requires wearing the VR headset to fully experience the immersive surrounding of the content. However, the novel viewing experience of VR video creates new challenges and requirements for conventional video authoring tools, which were designed mainly for working with normal video on a desktop display. Designing effective authoring tools for VR video requires intuitive video interfaces specific to VR.

This dissertation develops new workflows and systems that enable filmmakers to create and improve VR video while fully immersed in a VR headset. We introduce a series of authoring tools that enables filmmakers to work with video in VR: 1) Vremiere, an in-headset video editing application that enables editors to edit VR video entirely in the headset, 2) CollaVR, a networked system that enables multiple users to collaborate and review video together in VR, and 3) a set of techniques to assist filmmakers in managing and accessing interfaces in stereoscopic VR video without suffering depth conflicts. The design of these applications is grounded in existing practices and principles learned in interviews with VR professionals. A series of studies is conducted to evaluate these systems, which demonstrate the potential of in-headset video authoring.
\end{abstract}




\section{Dedication}

To Dad, Mom, and Phuong 


\section{Acknowledgements}

I would like to thank my Ph.D advisor Prof. Feng Liu. Over the course of five years, Feng had provided me with tremendous support, from mentoring me when I first started to motivating me through tough deadlines. Feng inspired me to find joy in doing research, which I believe is the greatest thing I have learned.

At the Maseeh college at PSU. Prof. Wu-chi Feng always encourages me to improve

my research and also inspires me to stay healthy. At the time of this writing, my personal record in the Deadlift is 2.3 times my body weight. I also want to thank my committee members Prof. Bart Massey and Prof. Miguel Andres Figliozzi, and my lab mates Long Mai, Hoang Le, and Simon Nicklaus. They provided helpful feedback and created an invigorating academic environment that I am proud to be a part of.

Outside of PSU, I am fortunate to work with Dr. Stephen DiVerdi and Dr. Aaron Hertzmann at Adobe Research. This collaboration has led to three exciting projects that form this dissertation. More than that, their knowledge and dedication have reinforced my dream of becoming a research scientist.

I am thankful to Mom, Dad, and my extended families for providing me with unconditional love. They always encourage me to pursue what I love most. I am also grateful to have Edmund Klein as a friend and an amazing life mentor. Ed taught me to appreciate life and to persevere when things get tough.

I also want to thank whoever invented cold-brew coffee.

Finally, I want to thank Phuong Do for always be there for me in the up and down of graduate school. Her keen interest in my research passions makes me believe I can change the world one small step at a time. Her unconditional support gives me the strength to carry on when my experiments fail. Her warm presence is a constant reminder that my life is filled with beauty and gratitude. I met Phuong when I first started graduate school and married her in my last year. I am so lucky. 
Table of Contents

Abstract i

Dedication ii

Acknowledgements iii

List of Tables vii

List of Figures viii

1 Introduction 1

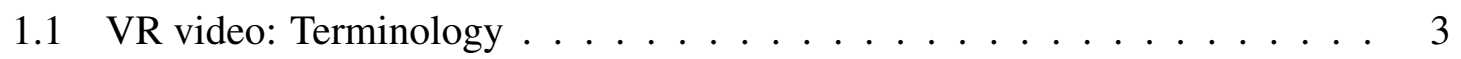

1.2 Challenges of VR Video Authoring . . . . . . . . . . . . . . 4

1.3 In-Headset Authoring Tools . . . . . . . . . . . . . . . . 6

1.4 Contributions . . . . . . . . . . . . . . . . 7

1.5 Overview ............................. 8

1.6 Prior Publications $\ldots \ldots \ldots \ldots$

2 Background and Previous Works 10

2.1 What Makes a VR Narrative? . . . . . . . . . . . . . . . . . 10

2.2 VR Video Editing . . . . . . . . . . . . . . . . . . . . . . 12

$2.2 .1 \quad$ Desktop-based video editing tools . . . . . . . . . . . . . . 12

$2.2 .2 \quad 360^{\circ}$ video navigation . . . . . . . . . . . . . . . . 12

2.2 .3 Editing tools for VR video . . . . . . . . . . . . . 13

2.3 VR Video Collaboration . . . . . . . . . . . . . . . . . . . . . . . . . . . . 14

2.3 .1 Collaboration in VR . . . . . . . . . . . . . . . . . . . . . . . 14

2.3 .2 Watching VR video together . . . . . . . . . . . . . . 14

2.3 .3 Collaborative video reviewing . . . . . . . . . . . . 15

2.4 Integrating UIs in Stereoscopic VR Video . . . . . . . . . . . . . 16

2.4 .1 Depth perception in stereoscopic VR video . . . . . . . . . . 16 
2.4 .2 Techniques to reduce depth conflicts . . . . . . . . . . . 17

3 Vremiere: In-Headset VR Video Editing 19

3.1 Motivation . . . . . . . . . . . . . . . . . . . . 19

3.2 VR Professional Interviews . . . . . . . . . . . . . . . . . 22

3.2 .1 Navigating footage . . . . . . . . . . . . 23

3.2 .2 Assembling cuts . . . . . . . . . . . . . 23

3.2 .3 Previewing edits . . . . . . . . . . . . . 23

3.2 .4 Titling . . . . . . . . . . . . . . . . . 24

3.2 .5 Review and annotation . . . . . . . . . . . . . . 24

3.3 In-Headset Editing Workflow . . . . . . . . . . . . . . . . . 24

3.4 The Vremiere System . . . . . . . . . . . . . . . . . 26

3.4 .1 Timeline editing . . . . . . . . . . . . . . 27

3.4 .2 Video browsing - visualization . . . . . . . . . . . . . 27

3.4 .3 Video browsing - comfort . . . . . . . . . . . . . . . 29

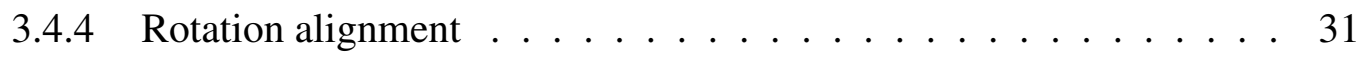

3.4 .5 Titling . . . . . . . . . . . . . . . . 33

3.4 .6 Bookmarking and reviewing . . . . . . . . . . . 34

3.5 User Study . . . . . . . . . . . . . . . . . . . . . 35

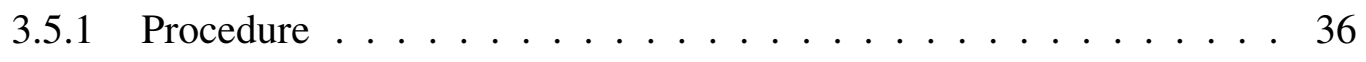

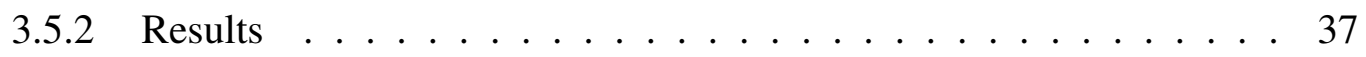

3.6 Discussion . . . . . . . . . . . . . . . . . . 40

3.6 .1 Requested features and limitations . . . . . . . . . . . . . . 41

3.7 Conclusion . . . . . . . . . . . . . . . . . 42

4 CollaVR : In-Headset Collaborative Video Reviewing 44

4.1 Motivation . . . . . . . . . . . . . . . . . . . . . 44

4.2 VR Professional Interviews . . . . . . . . . . . . . . . . . . 46

4.2 .1 Social awareness . . . . . . . . . . . . . . 47

4.2 .2 Common context . . . . . . . . . . . . . . 47

4.2 .3 View sharing . . . . . . . . . . . . . . . . 48

4.2 .4 Notetaking . . . . . . . . . . . . . . . . 49

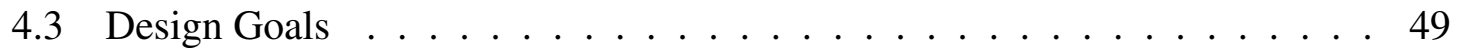

4.4 The CollaVR System . . . . . . . . . . . . . . . . . 50

4.4 .1 Awareness visualization ................. 51

4.4 .2 View sharing . . . . . . . . . . . . . . 54 
4.4 .3 Feedback recording and browsing . . . . . . . . . . 56

4.5 User Study . . . . . . . . . . . . . . . . . . . . . . . . 57

4.5 .1 Results . . . . . . . . . . . . . . . 60

4.6 Discussion and Limitations $\ldots \ldots \ldots$. . . . . . . . . . . . . . . 65

4.7 Conclusion . . . . . . . . . . . . . . . . 66

5 Depth Conflict Reduction for Interfaces in Stereoscopic VR Video 67

5.1 Motivation . . . . . . . . . . . . . . . . . . 67

5.2 Depth Conflicts in VR Video Interfaces $\ldots \ldots$. . . . . . . . . . . . . . 69

5.3 Techniques Considered . . . . . . . . . . . . . . . . 70

5.3 .1 Baseline . . . . . . . . . . . . . . . . 71

5.3 .2 Dynamic Depth . . . . . . . . . . . . . 71

5.3 .3 Halo Blur . . . . . . . . . . . . . . . . . . 74

5.4 User Study . . . . . . . . . . . . . . . . . . . . 75

5.4 .1 Tasks . . . . . . . . . . . . . . . 75

5.4 .2 Experiment design \& procedure . . . . . . . . . . . . . 77

5.4 .3 Results . . . . . . . . . . . . . . . . . . . 78

5.5 Discussion and Limitations $\ldots \ldots \ldots$. . . . . . . . . . . . 80

5.6 Conclusion $\ldots \ldots \ldots \ldots \ldots$. . . . . . . . . . . . . 82

$\begin{array}{llr}6 & \text { Conclusion } & 83\end{array}$

6.1 Summary of Contributions . . . . . . . . . . . . . . . . 83

6.2 Future Directions . . . . . . . . . . . . . . . . . . 84

6.2 .1 Interactivity in VR video . . . . . . . . . . . . . . . 84

6.2 .2 VR video as a collaboration space . . . . . . . . . . . . . . . . 84

6.2 .3 Data-driven VR video authoring . . . . . . . . . . . . . . . . 85

\begin{tabular}{lr}
\hline Bibliography & 87
\end{tabular} 


\section{List of Tables}

Table 3.1 Participants' demographics . . . . . . . . . . . . . . . . . . . 35

Table 3.2 Summary of responses to the question "I feel this editing operation is intuitive and easy to learn" (D: disagree, N: neutral, A: agree). . . . . 38

Table 3.3 Summary of responses to the question "I feel this widget is useful for my editing task in VR" (D: disagree, N: neutral, A: agree). . . . . . . . 39

Table 3.4 Summary of responses to the question "I prefer this editing operation in this VR system as compared to my current workflow". (D: disagree, $\mathrm{N}$ : neutral, A: agree) . . . . . . . . . . . . 40

Table 4.1 Results of the study. The headset view similarity of group 1 was computed by averaging the view similarity of each pair in the group. . 62

Table 5.1 Brief descriptions of the video materials used in the study. . . . . . . 76

Table 5.2 Subjective questionnaire. Participants reponded with a 7-point Likert scale, ranging from very difficult to very easy (Q1,Q2) and not at all to very much $(\mathrm{Q} 3, \mathrm{Q} 4, \mathrm{Q} 5) . \ldots \ldots . \ldots 77$ 


\section{List of Figures}

Figure 1.1 Illustration of viewing VR video and two common formats of VR video. (a) VR video means $360^{\circ}$ video viewed within a headset. The $360^{\circ}$ video of the ski lift is texture mapped to a viewing sphere. The viewer is at the center of the sphere and can control the field of view (the red box) using head rotation. (b) VR video is often represented as monoscopic equirectangular images ( (C) Ábaco Digital Zaragoza).

(c) VR video can also be stereoscopic, in which a pair of left-right equirectangular images is captured. To convey depth perception, VR headsets typically render the upper image to the viewer's left eye and the lower image to the right eye. (ङ Kevin Kunze) . . . . . . . . . . 2

Figure 2.1 A common video production pipeline. This dissertation focuses on the post-production stage, which includes various video authoring workflows such as video editing or video reviewing. . . . . . . . . . 11

Figure 2.2 (a) Both eyes converge on a Rubik cube but, due to binocular disparity, each eye views the cube slightly differently. The human visual system interprets this difference as depth. (b) When viewing stereoscopic VR video, the viewer sees two different video views in each lens and, therefore, can perceive depth. (Notice the male actor is presented in the right view but not in the left view). (c) Kevin Kunze . . . . . . . . 16

Figure 3.1 The same shot, viewed in a headset versus on the screen. VR views look very different than on the desktop screen, and provide a much stronger sense of immersion. (C) TOYO TIRES JAPAN . . . . . . . . . . 20

Figure 3.2 The Vremiere interface contains a video view in the background (A) and floating UI components such as the timeline (B), editing tracks (C) and editing widgets (D). By using familiar mouse and keyboard interaction, an editor can edit and view the $360^{\circ}$ video directly inside the VR headset. . . . . . . . . . . . . . . . . . . . . . . . . 25 
Figure 3.3 Left: the Little Planet is shown in a small window above the timeline to aid in-headset video navigation. Right: close-up view of the visualization; it can be used both as a minimap and a compass of the $360^{\circ}$ image. As noted by the yellow arrow, the user can quickly spot an interesting event outside her field of view (indicated by the yellow fan at the center) that would be otherwise difficult to find. . . . . . . . . 27

Figure 3.4 Our system dynamically contracts a pair of vignettes in front of the user's eyes based on the perceived motion in the video, to help reduce discomfort during video playback or scrubbing. Note, on the left, the default diameter of the vignette is $120^{\circ}$, which corresponds to no vignetting. (C) Ábaco Digital Zaragoza . . . . . . . . . . . . . . . . . 29

Figure 3.5 A: an example of a jarring cut in VR video. The cut transitions from the ski lift to an empty view of the mountain in the ski_2 video. B: Our Rotation Alignment tool is shown above the timeline. In the close-up view, the user can visualize how the cut transitions from one clip to another given a specific viewpoint in the video (visualized by yellow bars); she can also directly drag on the clips to align events before and after a cut, thereby helping the viewer to follow key elements in the video. Here, the Ripple Rotation mode is shown currently as an Unlink toggle. C: after rotation, the skier in clip $s k i \_2$ is aligned to the previous shot, resulting in a much better cut. (C) Ábaco Digital Zaragoza 31

Figure 3.6 Left: On desktop, adding 2D text or images to a $360^{\circ}$ video is often unintuitive. Right: in the headset, our system provides a natural view of the image (Sample Logo) and enables users to directly place it anywhere in the scene. (C P J Orravan . . . . . . . . . . . . . . 33

Figure 3.7 The user can directly click on the video to add markers. These markers are visualized on the timeline and can be reviewed quickly using keyboard shortcuts. (C) Jacob Phillips . . . . . . . . . . . . . . 34

Figure 4.1 (A): In-headset views of two CollaVR users watching a video together. The clients are connected through a local network. In the headset, users see visualizations of each other's viewport. User 2 (purple) circles a stitching artifact on the video and the drawing immediately appears on user 1's (red) screen. (B): The timeline interface includes features to support communication, view sharing, and notetaking for VR video. . . . . . . . . . . . . . . . . 44 45 
Figure 4.2 Left: Our system renders the viewport of a collaborator as a colorcoded rectangle. The extent of the rectangle depicts the field of view. The 3D cursor pointer is also rendered inside the viewport, so users can point to a video scene element. Middle and Right: When the viewport travels outside the current field of view, an arrow appears on the periphery to provide a directional cue toward the off-screen position. 51

Figure 4.3 Illustration of spatialized voice chat between user 1 and user 2. As

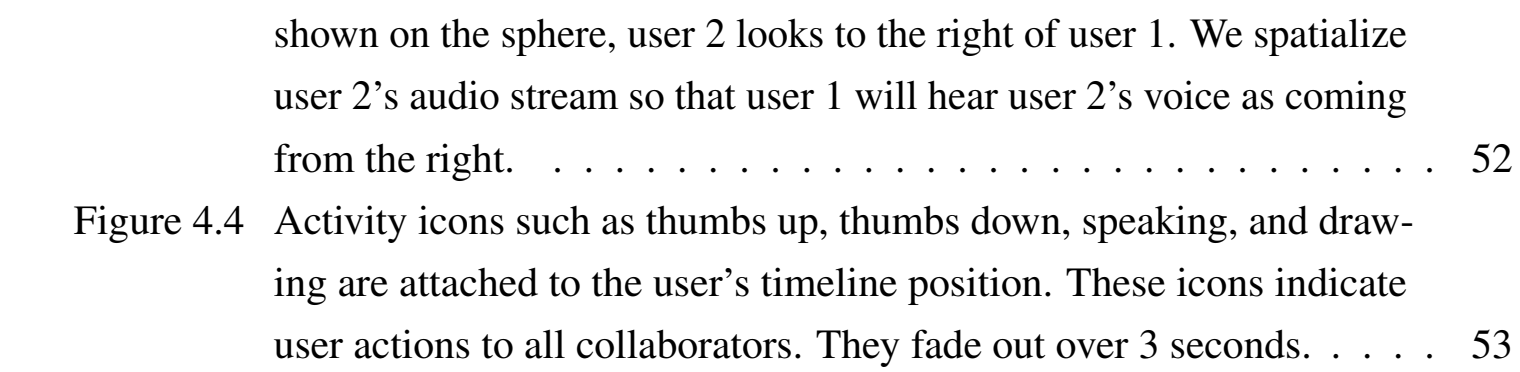

Figure 4.5 View sharing tools. When "Peek" is enabled, we display color-coded thumbnails showing each user's (e.g., \#2 and \#3) current video view. The user can further click "follow in time" or click on the thumbnail to trigger different view sharing modes. . . . . . . . . . . . . . 55

Figure 4.6 Slave visualization. The master user looks at the bicycle rider (right). \begin{tabular}{|l|}
\hline The slave user (left) uses "Slaving' to watch the master user's view. \\
\hline In this mode, the slave's own view is dimmed and a reduced-size \\
\hline copy of the master full field of view is rendered opaquely on top \\
\hline (middle). This allows the slave user to observe the master's actions \\
\hline while retaining peripheral self-motion perception. . . . . . . . . . 57
\end{tabular}

Figure 4.7 Recorded feedback is listed on the "Notes" panel, color-coded by

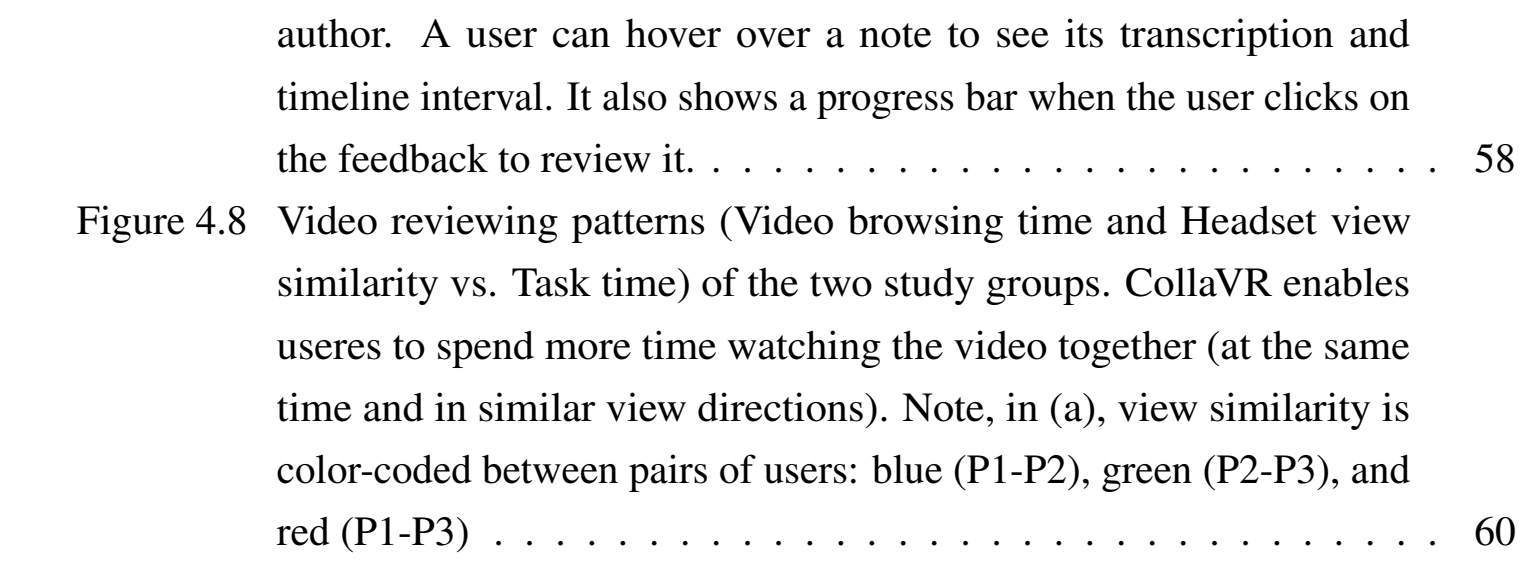


Figure 4.9 Communication patterns, shown as fraction of implicit and explicit references uttered by study participants. The numbers above the chart show the total number of references made in that condition. . . . . . 63

Figure 4.10 Results of the post-task questionnaire (a) and subjective feedback

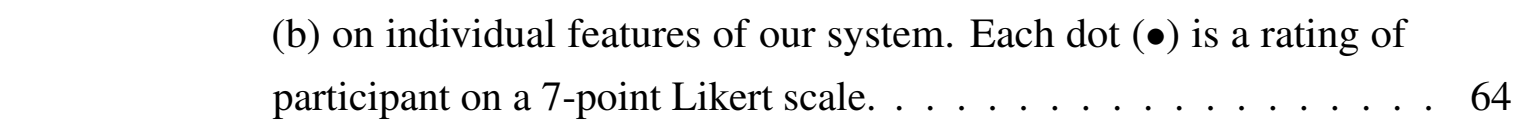

Figure 5.1 Depth conflict illustration. An interface (the video player) overlays a video object (the actress) but is actually behind her in depth. Left: The resulting graphics are uncomfortable to view in VR (e.g., the areas behind the text 00:01 in the insets are different between the left and right views). Viewers may also experience difficulty changing focus between the interface and the video. Right: illustration of the conflicting depth cues perceived by the same viewer in the same view. (c) Kevin Kunze . . . . . . . . . . . . . . . . . . . . . . . . . . . . 68

Figure 5.2 Overview of Dynamic Depth. (A) We pre-process the input video to find feature points and left/right disparities (e.g., green lines on the actress) (B) Features points are mapped to the VR view and shown as the green dots (only for illustrative purposes). Dynamic Depth estimates the perceived depth of the video based on these points. It detects when depth conflict occurs by comparing the depths between the UI and the video. (C) Dynamic Depth moves the UI closer to the viewer to reduce depth conflicts. Notice in the insets that the areas around the interface's corner are more geometrically consistent compared to the same scene in Figure $5.1 \mid$ (๑) Kevin Kunze . . . . . . 71

Figure 5.3 Halo Blur blurs the video content around the UI. Insets: compared to the same scene in Figure 5.1, the current scene is still not geometrically consistent. However, the blur effects mask high-frequency spatial information in the video images and makes the details from the UI clearer. (c) Kevin Kunze . . . . . . . . . . . . . . . . . . 75

Figure 5.4 (Left) Summary of participants' ratings to the subjective questionnaire in both tasks. (Middle and Right) Task time and task error summary of the Search task. . . . . . . . . . . . . . . . . . . 78 


\section{Introduction}

Virtual Reality (VR) technology has been around for decades. In 1968, Ivan Sutherland demonstrated the first VR system and paved the way for modern VR technology [106]. The central component of the system is a head-mounted display (HMD) that a user can wear like a headset. The display is connected to a computer, which tracks its orientation and position and updates the rendered images in the display accordingly. In this way, the system can create the illusion that the viewer is immersed in a three-dimensional (3D) virtual world. Another defining feature of VR is interactivity. Equipped with tracked controllers, a user can not only immerse in a virtual world, but also manipulate it interactively [15]. This unique combination has led to interesting applications of VR such as military training, architecture visualization, and telepresence. Most recently, VR technology has become sufficiently mature to attract both commercial and public interests [98].

Of central to the recent adoption of VR is the development of cinematic VR experiences [75]. Cinematic VR allows a viewer to experience virtual narratives that have been preproduced, either through rendered graphics or specialized camera rigs, whereas traditional VR requires real-time graphics rendering capabilities. Thus, cinematic VR can be shared on a wide variety of publishing platforms and VR devices. Computer users of all ages can enjoy immersive experiences as easy as watching a video.

Indeed, one type of cinematic VR content gaining popularity is $V R$ video. Virtual reality video specifically means $360^{\circ}$ video viewed within a VR headset. Figure 1.1 a illustrates a typical setup for viewing VR video. These videos are often captured using spherical camera rigs that can capture $360^{\circ}$ pictures and audio. Unlike traditional cinema, where the 
video is bounded by the movie screen, watching a VR video means the viewer is inside the movie and can freely look around and experience the story. It allows filmmakers to consider dramatically new narrative tools: What if the viewer could experience what it is like to swim with sharks in ocean or climb Mount Everest? Filmmakers are embracing VR video as an artistic medium to unlock unprecedented potential in immersive media.

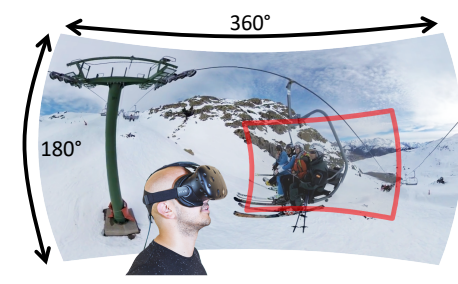

(a) Viewing VR video

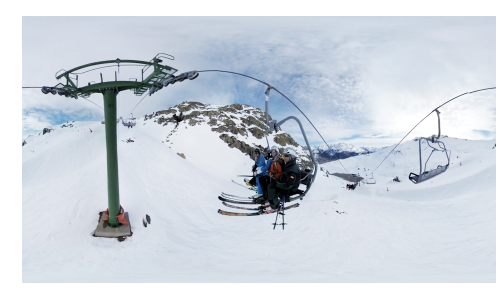

(b) Monoscopic VR video

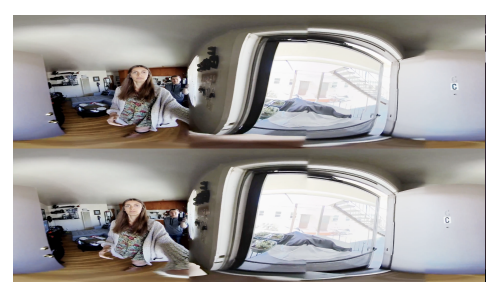

(c) Stereoscopic VR video

Figure 1.1: Illustration of viewing VR video and two common formats of VR video. (a) VR video means $360^{\circ}$ video viewed within a headset. The $360^{\circ}$ video of the ski lift is texture mapped to a viewing sphere. The viewer is at the center of the sphere and can control the field of view (the red box) using head rotation. (b) VR video is often represented as monoscopic equirectangular images ( Ábaco Digital Zaragoza). (c) VR video can also be stereoscopic, in which a pair of left-right equirectangular images is captured. To convey depth perception, VR headsets typically render the upper image to the viewer's left eye and the lower image to the right eye. (C Kevin Kunze)

An important question that must be asked of every new artistic medium is, How can users produce content for such medium? Virtual reality video presents content in a different nature when compared to traditional cinema. Thus, to produce good video, filmmakers need to explore, adapt, and sometimes break the creative boundaries of traditional filmmaking [75]. A key requirement to support these creative needs is video authoring tools. Just as hammers and chisels help sculptors refine raw stones, video authoring tools offload the burden of manipulating digital images and sound so filmmakers can focus more on constructing the desired narrative. More importantly, effective tools can foster creativity by allowing filmmakers to try new ideas and evaluate them quickly [101].

The emphasis of this dissertation is on VR video authoring. Specifically, this work focuses on two research questions: 
1. How can VR authoring tools help filmmakers create and improve VR video?

2. What new interface mechanisms are needed to support video authoring in VR?

Research in the narrative structure of VR video has been undertaken since the 1990s, and the general consensus is that VR production can draw upon long-established filmmaking practices [8, 4, 75]. In filmmaking, post-production refers to processes and workflow that occur after shooting video footage. Video interaction is central to that workflow. To that end, film professionals typically spend significant time and effort in activities such as video editing, special effects, and video reviewing.

Video authoring tools that aid post-production have been well studied [99, 78]. However, existing tools and workflows of regular video do not translate well to VR video. Current video interfaces are designed mainly for interacting with normal field-of-view (NFOV) video ${ }^{1}$ on the desktop, whereas VR video is intended to be experienced with a VR headset. This discrepancy poses significant challenges to many video authoring tasks. To better comprehend the problem, it is important to understand the differences between VR and NFOV video, current practices in VR video production, and associated challenges.

\subsection{VR video: Terminology}

Throughout the text, we use the terms VR video and $360^{\circ}$ video interchangeably. Virtual reality video can be either monoscopic or stereoscopic. For brevity, we refer to monoscopic VR videos as $V R$ videos because they are by far the most commonly available [73], and explicitly mention stereoscopic VR videos otherwise.

A $360^{\circ}$ video is often recorded using panoramic camera rigs. A typical setup contains multiple cameras that can capture the full sphere around the camera center. The recorded images are then processed, aligned, and stitched in software to produce the final $360^{\circ}$ video

\footnotetext{
${ }^{1}$ The term NFOV video is first coined by $\mathrm{Su}$ et al. [105] to distinguish normal videos from VR videos. These videos are viewed on non-immersive displays such as desktop monitors or mobile devices.
} 
[107]. Figure $1.1 \mathrm{~b}$ shows a sample frame from a monoscopic $360^{\circ}$ video; the frame is represented in equirectangular projection - a common format to display $360^{\circ}$ image on desktop computers.

Capturing VR video is a growing application field. There are other VR video formats. Stereoscopic VR video (Figure 1.1 ), which provides a much greater sense of immersion than monoscopic video, is becoming increasingly available due to many recent advances in camera and video technology [2, 76, 58]. When viewed in a VR headset, these videos show slightly different $360^{\circ}$ images for the left and right eyes, so the viewer can perceive objects in the video at different depths. More complex hardware that can capture light-field images are being explored, but have not yet become commercial products. This dissertation focus only on monoscopic and stereoscopic VR video.

For immersive viewing experiences, the most common way to view is using the VR headset. Virtual reality headsets are head-mounted displays. The first HMD was developed in 1968 [106]. Nowadays, HMDs are lightweight, powerful, and relatively comfortable for everyday use. Although VR video can also be viewed with other display technologies such as CAVE, desktop, or mobile devices [73], they either require overly complex setup or do not provide a comparable immersive quality.

\subsection{Challenges of VR Video Authoring}

Watching video in VR gives viewers a unique experience dramatically different from standard NFOV video. This novel viewing experience, however, manifests into many new challenges and requirements for post-production workflows. The differences and associated challenges include:

1. Virtual reality video is immersive. A person watching VR video for the first time often laughs with joy, screams in terror, or reaches for something in mid-air. Thus, VR video requires editors to carefully consider how their edits will affect the experience 
in the headset. For example, the time it takes to absorb and experience VR often affects the pacing of cuts. Editors must also deal with the potential strain, fatigue, and nausea [57] of VR for both the editor and the intended viewer. At present, video editors must follow an onerous workflow, alternating between editing on the desktop and then putting on a headset to review the edit.

2. In VR, a viewer sees only a portion of the video at any time. This aspect of VR video is intrinsically different than NFOV video, in which a viewer always see the whole video frame. This difference challenges conventional editing techniques, such as zoom or jump cut, that rely on knowing where the viewer will look. Applying conventional techniques in VR video can often lead to confusing or jarring experiences [17].

3. Wearing a VR headset also blocks views of the outside world, making interpersonal interaction and communication around the video difficult or impossible. Filmmakers frequently collaborate and review video together - a process often termed dailies. Video editors, producers, and clients watch the video together, discuss, and note changes and improvements for the editing [5]. However, in VR, collaborators cannot see each other. Two participants in the same room cannot easily point to a specific element in a video. Because each viewer effectively has their own video player, even synchronizing timing and view direction are difficult. The headset also makes it difficult to use notetaking devices such as paper or keyboard.

4. VR video creates new interface needs in video. Virtual reality video interaction requires support for both temporal (time in the video) and spatial (where to look) contexts, which renders existing techniques for regular video unsuitable in certain scenarios. For example, when searching for key video moments in VR, a user can quickly get lost because he or she cannot look at the whole $360^{\circ}$ scene.

Overall, the difficulties that arise when authoring VR video can be summarized by two 
main themes. First, authoring VR video requires working with the video in the headset, but current tools are designed only for the desktop computer. Second, interacting with VR video creates new, largely unexplored interface needs, such as navigating the video in both time and view direction.

In human-computer interaction (HCI) research, designing for effective interaction requires bridging both the gulf of execution (i.e., the gap between a user's goal and means to execute that goal) and the gulf of evaluation (i.e, how hard it is for the user to interpret the system output) [52]. It is clear that both gulfs are significant problems for filmmakers, who currently must interact with VR video using tools designed for NFOV video, alternating between desktop authoring tools and the headset display to view the VR content. There is a clear benefit to more streamlined and direct interaction of VR video.

\subsection{In-Headset Authoring Tools}

To address the presented challenges, this work aims to develop authoring techniques and workflows that allow filmmakers to work with video directly in the VR headset. My research focuses on two typical post-production workflows: video editing and collaborative video reviewing. The diverse range of video interactions that both workflows require allows a broad study of how users can interact with video in an immersive environment.

My research introduces a series of systems and interaction techniques to demonstrate in-headset video authoring: video editing, collaborative video reviewing, and interacting with stereoscopic VR video.

Video editing. Virtual reality video editing is still done primarily in the traditional 2D desktop environment with limited capability to view VR content or directly manipulate the video in an intuitive way. As a result, editors must alternate between editing on the desktop and previewing with the headset, which is tedious and interrupts the creative process. In response, we develop Vremiere, an in-headset video editing application. Vremiere allows 
a video editor to edit VR video while fully immersed in the headset. This advantage is important because it enables a seamless workflow in VR, which is key to facilitating the creative process around VR video.

Collaborative video reviewing. Taking editing a step further, what if multiple filmmakers want to work together in VR? Collaboration and review are integral parts of the conventional filmmaking process. However, collaboration is difficult when wearing a VR headset that blocks all views of the physical world. To address this problem, we develop CollaVR, an application that enables multiple users wearing headsets to review a VR video together. CollaVR introduces a set of techniques that support multiple users to watch a VR video, exchange feedback, and take notes together without being hindered by the VR headsets.

Interacting with stereoscopic VR video. Vremiere and CollaVR mainly focus on monoscopic VR video. Beyond monoscopic, stereoscopic VR videos convey an additional sense of depth and can potentially enhance the VR experience. However, simultaneously displaying video interfaces with stereoscopic video content can cause undesirable depth conflicts. This problem prevents users from efficiently using video applications in VR, including authoring tools. To address this problem, we develop two techniques to assist users in using video applications in stereoscopic VR video without suffering from depth conflicts.

\subsection{Contributions}

Overall, the primary contributions of this work are to 1) understand and address specific design requirements in VR video authoring, and 2) develop new systems and interaction techniques that enable filmmakers to create and improve VR video while fully immersed in the VR headset. We validate these systems through a series of evaluation studies with VR film experts and VR users to solicit feedback and important insights for future development. 
In combination, these components demonstrate the potential for in-headset authoring for VR video, particularly in supporting the growing creative needs around this medium.

These contributions are made possible by incorporating knowledge and technologies from various fields: video analysis techniques from computer vision to process and extract useful information from the video, rendering and visualization techniques from computer graphics to enhance video interactions in VR, and understanding of human perception, cinematography, and video interfaces to inform the design of video authoring tools in VR.

Thesis statement: Perform video authoring tasks directly in the headset—and having tools specific to VR-would significantly improve the post-production process of VR video.

\subsection{Overview}

The rest of this dissertation is organized as follows. In Chapter 2, we review the literature on research, technologies, and commercial products used in supporting VR video authoring. In Chapter 3, I present the exploration, development, and evaluation of an in-headset VR video editing interface. We introduce the Vremiere system, which allows video editors to edit VR video in the headset, and a series of interaction and visualization techniques designed for editing video in VR. Chapter 4 moves the techniques a step further and explores video authoring in the context of multiple users. Specifically, we focus on the collaborative video reviewing workflow and explore how multiple users can collaborate and review video together in VR. Chapter 5 considers stereoscopic VR video. We explore the depth-conflict problems that arise from user interfaces (UIs) in stereoscopic VR video and demonstrate two techniques to reduce the conflicts. Finally, in Chapter 6, which concludes this dissertation, we propose future research directions for in-headset video authoring. 


\subsection{Prior Publications}

The results in this dissertation have been published in or submitted to ACM conference proceedings. I am the primary author on all of these publications. However, these projects could not have been completed without the generous support from my advisor, Dr. Feng Liu, and my collaborators, Dr. Stephen DiVerdi and Dr. Aaron Hetzmann. The work on Vremiere in Chapter 3 was published in CHI 2017 [84]; the collaborative reviewing system CollaVR in Chapter 4 was published in UIST 2017 [83]; and the work on depth-reduction techniques in Chapter 5 is submitted to CHI 2018 and is currently under review. 


\section{Background and Previous Works}

This dissertation intersects with diverse areas of HCI and VR research. In this chapter, we first review the literature on the narrative structure of VR (Section 2.1), an important concept to understanding VR video authoring. We will then survey state-of-the-art techniques for supporting VR video authoring and focus the discussion on two important workflows: video editing (Section 2.2) and collaborative video reviewing (Section 2.3). This chapter concludes by discussing perceptual issues that arise when designing interfaces for stereoscopic VR video and the related literature (Section 2.4).

\subsection{What Makes a VR Narrative?}

Virtual reality technology has been around for decades. Although most research in VR focused on software and hardware aspects, early works recognized the potential of VR as an artistic medium. Bates explained the fundamental driving force that transforms VR from a technology to an art form is the ability to transport the viewer to "go anywhere and do anything" [8]. Through a comparative analysis between VR and other artistic media such as literature, film, and game, Aylett et al. also showed that VR should be considered an entertainment medium in its own right [4]. Both works argued that practitioners need to develop a distinctive VR narrative form with its own production techniques and grammar.

Narrative is formally defined as "a representation of connected events and characters that has an identifiable structure, is bounded in space and time, and contains implicit or explicit messages about the topic being addressed" [60]. In filmmaking, narrative can be understood as the story the audience perceives when watching the video. 
The interest of the filmmaking community in VR video production seems to have followed the vision of Bates and Aylett. Compared to computer-generated graphics content, VR video is made from video captured images. When a person watches VR video, the content unfolds linearly, just as it does in traditional films, but the viewer still has the freedom to look around-a unique immersive characteristic of the VR medium. The combination of similarity and particularity to the traditional film medium is important for VR filmmakers. They can not only adapt long-established filmmaking techniques to VR video, but also experiment with new techniques to turn it into a distinctive narrative form [75].

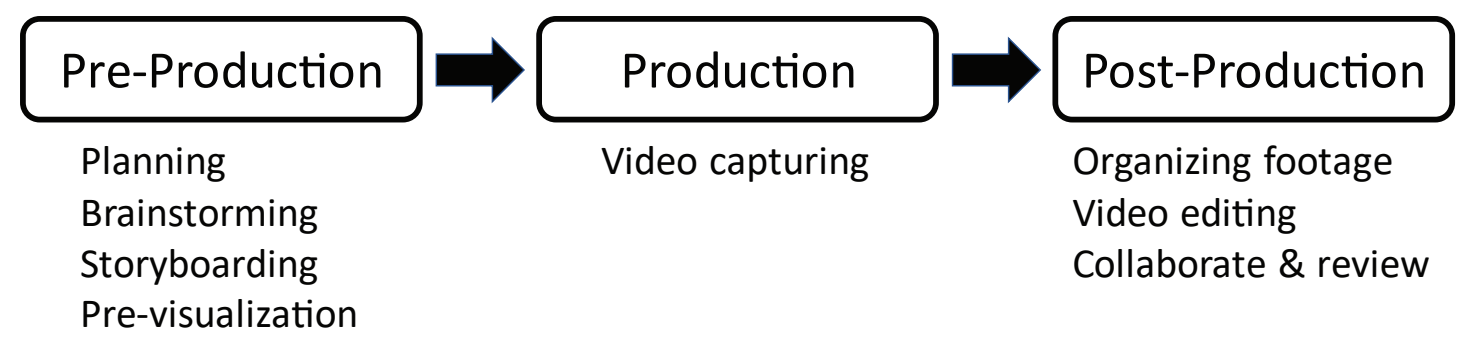

Figure 2.1: A common video production pipeline. This dissertation focuses on the postproduction stage, which includes various video authoring workflows such as video editing or video reviewing.

Traditional filmmaking techniques typically follow the standard video production pipeline (Figure 2.1). The pipeline consists of multiple stages, each of which are often supported by a multitude of software tools. In the first stage, the video's narrative is conceived through storyboarding and pre-visualization tools [47]. In the production stage, crews use omnidirectional camera technologies to capture $360^{\circ}$ video footage [2]. Finally, in post-production, various video authoring tasks such as editing and reviewing are carried out. These tasks are central to turning the raw video clips into the desired narrative. Editing techniques are executed in software to rearrange the plot, adjust the pacing, or intensify certain emotions [67]. Multiple stakeholders frequently conduct group reviews to evaluate and improve the edits [5]. In combination, these tasks allow filmmakers to create, evaluate, and refine raw VR video content into a comprehensible narrative form. 
These studies and observations suggest video authoring tools play an important role in realizing the narrative of VR video. This dissertation aims to support video authoring by exploring and developing these tools in VR. Next, we survey related works on technologies for supporting VR video authoring.

\subsection{VR Video Editing}

\subsubsection{Desktop-based video editing tools}

Video editing is the craft of combining raw video footage into a coherent sequence of events. Professional editors have developed standard cinematic rules and guidelines [31], but video editing still remains a challenging task. Video editors use established software tools to support their tasks. For example, there are tools that support general purpose editing (e.g., Adobe Premiere, Apple Final Cut, Sony Vegas), visual effects (e.g., Adobe After Effects, Nuke Studio), and color grading (e.g., DaVinci Resolve). In addition, researchers have developed automatic methods to support editing a wide range of video contents such as interview, tutorial, lecture, or egocentric video [21, 10, 24, 71, 110]. These authoring tools have mostly focused on NFOV video and assumed the audience could view the whole video frame. However, a viewer of VR video sees only a portion of the video and must actively explore the $360^{\circ}$ scene. This difference is challenging filmmakers to explore new ways to edit VR video. Thus, developing effective authoring tools for filmmakers can have substantial impact on their creative process [101].

\subsection{2 $360^{\circ}$ video navigation}

In video editing, it is important to support effective video navigation [99]. Because a $360^{\circ}$ video viewer shows only a portion of the scene at once, interfaces are needed to manipulate both time and viewing direction. Researchers have developed hand gestures for $360^{\circ}$ video navigation, for CAVE-like environments [94], and for VR headsets [89]. These gestures 
are used for basic controls such as play, pause, fast forward, rewind, zoom, and pan. My work focuses on more advanced editing operations. The intended users are video editing professionals who typically have deep expertise in keyboard shortcuts and efficient mouse interactions.

Conventional timeline-based video players have been extended to browse $360^{\circ}$ video on the desktop. The FlyAbout system [56] provides a map-based interface that lets users browse and explore spherical video similarly to Google StreetView. Neng et al. [82] treated $360^{\circ}$ video as "hypermedia" that can be annotated with URLs or text, and supported exploration of spherical content with features such as panning, navigation compass, and panoramic thumbnails. To this end, Vremiere (Chapter 3) also enhances the timeline with various navigation aids and extends it into a what-you-see-is-what-you-get (WYSIWYG), in-headset editing interface.

\subsubsection{Editing tools for VR video}

Recently, commercial tools have been updated to support VR video editing. The 2015.3 release of Adobe Premiere included a "virtual headset" view for spherical video. The user can click and drag on this view to change the viewpoint. The Skybox VR Player by Mettle is a Premiere plug-in that streams video to the Oculus Rift headset. Several $360^{\circ}$ cameras also come with software such as the THETA+ Video app, Nikon KeyMission 360/170 Utility, and the Samsung Gear 360 Action Director, that help with light editing and export. However, the user still cannot edit directly in the headset and must switch between the headset and the desktop interface. Skybox also provides a mode to show the entire Premiere desktop interface within the headset, but this does not provide WYSIWYG editing: the user must switch back to the viewer mode for review. Moreover, a user may have difficulty interacting with such a complex interface within a headset, especially because current headsets are relatively low resolution. In contrast, Vremiere (Chapter 3) supports direct viewing of the 
video together with editing and provides a minimal set of controls for the most important in-headset editing tasks.

\subsection{VR Video Collaboration}

\subsubsection{Collaboration in VR}

Research on VR collaboration primarily focused on 3D environments [9], and less thoroughly explored VR video. For example, the CU-SeeMe VR system [45] enables teleconferencing in "desktop VR" (a 3D virtual environment viewed on a desktop computer monitor, similar to a modern first-person video game), including an early form of spatialized audio voice chat. Fraser et al. [40] explored visualizations that support awareness in VR collaborations. For collaborative analytic tasks, Cordeil et al. [29] found that VR headsets can be good alternatives to CAVE systems and can support both co-located and remote collaboration. Perhaps most closely relevant to CollaVR (Chapter 4), Henrikson et al. [47] proposed a storyboarding system to allow an artist and a filmmaker to plan VR stories together, though those authors did not focus on simultaneous headset viewing scenarios. In contrast to these works, my research focuses on the review stage of video production, in which CollaVR provides tools designed specifically for fine-grained collaboration on VR video.

\subsubsection{Watching VR video together}

At the core of collaborative video review is the social act of watching a video together, but watching video in a VR headset is normally an isolated experience. Recent explorations have experimented with capturing and rendering humanoid avatars in VR, varying fidelity from simplified 3D models as seen in the Facebook 360 demo [93] to textured meshes from full body scans [77]. Avatars can convey body language and a sense of co-presence but require specialized capture equipment, such as depth cameras [68]. Moreover, avatars do not necessarily support the fine-grained collaboration required for film production. For example, 
they may not accurately convey where other participants are looking or pointing. McGill et al. found users are unsure whether to look at the avatars or the video, and the lack of shared cues about where the other participants were looking reduced their enjoyment [77]. In contrast, we designed my awareness visualizations for professional users to review video: the visualizations convey instantly what other people are doing and where they are looking. These nonverbal cues are important for video reviewing because they help collaborators refer to objects and ground conversation quickly [46]. Thus, my visualization could also aid social viewing.

\subsubsection{Collaborative video reviewing}

Collaboration, review, and feedback exchange are important processes in many authoring activities, including 3D design [111] and document authoring [118]. Within the space of video, some previous works focused on asynchronous review of conventional NFOV video. Phalip et al. [90] described a remote reviewing system for film scores. Pavel et al. [87] described a system that allows collaborators to record and exchange feedback and include video recording and browsing features to make asynchronous collaboration similar to in-person review. These systems' interfaces are feature rich and mainly designed to be used on a desktop computer. Although these techniques could be used in VR, this work emphasizes issues central to the problem of in-headset collaboration for VR video. To this end, we focus on synchronous review and address the issues of awareness, synchronization, and notetaking specific to the in-headset experience. Some commercial review systems such as Lookat.io [69] support adding annotations to VR video on a web browser but do not allow users to review or discuss in real-time in VR headsets. 


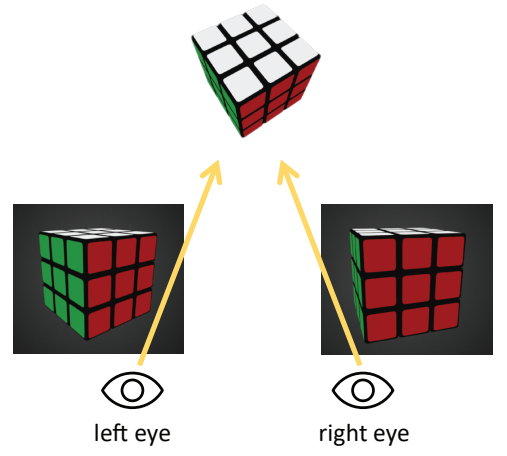

(a) Binocular vision

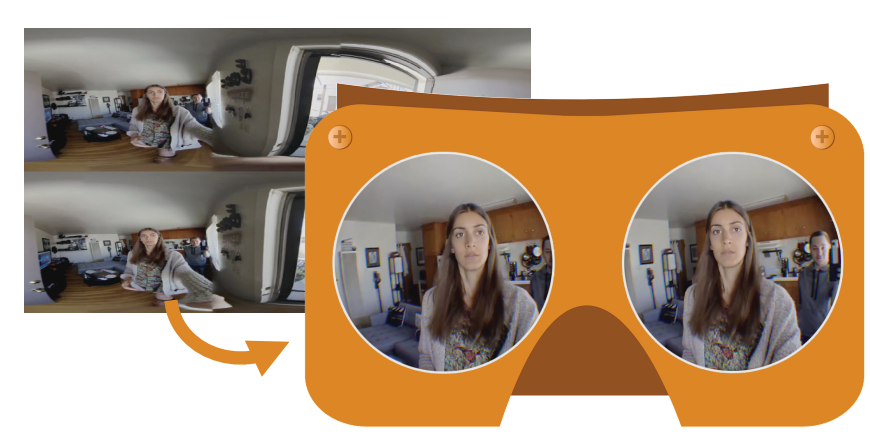

(b) Stereoscopic VR video viewed in a VR headset

Figure 2.2: (a) Both eyes converge on a Rubik cube but, due to binocular disparity, each eye views the cube slightly differently. The human visual system interprets this difference as depth. (b) When viewing stereoscopic VR video, the viewer sees two different video views in each lens and, therefore, can perceive depth. (Notice the male actor is presented in the right view but not in the left view). (C) Kevin Kunze

\subsection{Integrating UIs in Stereoscopic VR Video}

\subsubsection{Depth perception in stereoscopic VR video}

Monoscopic VR video, albeit immersive, lacks binocular depth perception. Most consumer VR headsets convey depth perception through binocular vision. Specifically, a VR headset has two lenses, each of which renders two computer-generated images slightly differently, one for each eye (Figure 2.2a). The differences in the projected images on the viewer's eyes are called disparity. The human visual system can interpret disparity information as depth [92].

Stereoscopic VR video conveys depth perception by capturing two slightly different images in each frame. These images are then projected to the VR lenses (Figure 2.2p). When viewing these images in the VR headset, the viewer can perceive depth.

However, there is an important distinction between the perceived depth from stereoscopic VR video and that from computer-generated graphics. Stereoscopic video content is captured using a camera rig and shown in the rendering pipeline simply as pairs of flat images. In contrast, 3D objects, UIs, or avatars are complex polygons processed in a full rendering 
pipeline. Consequently, when both types of content are rendered together, the stereoscopic depth from VR video and the computer-generated graphics might not be compatible and may conflict. An implication of this incompatibility is that video interfaces of authoring tools may cause undesirable problems in VR video. This dissertation explores this issue in Chapter 5 and introduces techniques to reduce depth conflicts so interface elements can be used in stereoscopic VR video.

\subsubsection{Techniques to reduce depth conflicts}

Visual discomfort induced by stereoscopic displays in VR HMDs is an active research area. Most work has focused on the vergence-accommodation conflict (VAC). Established solutions to this problem include rendering virtual content within a comfortable parallax zone or using novel hardware displays. For a detailed discussion, please refer to recent surveys [59, 50]. Besides vergence and accommodation cues, the human visual system can perceive depth from other cues such as occlusion, texture, or motion. Even when VAC is resolved, depth cues can conflict and cause discomfort [65]. This dissertation focuses on addressing a few specific depth conflicts that arise in VR video interfaces.

Depth conflict problems have been investigated in other stereoscopic media. In film production, the window violation problem occurs when a video object is seen as in front of the screen but is clipped by the edge of the screen [117]. However, window violation is not a problem in VR HMDs [42]. Subtitles placed on top of stereoscopic video can also cause depth conflicts [18, 64]. Video editors can place subtitles closer to the viewer during production, but they are still problematic in live-broadcast stereoscopic television [102]. Using subtitles in VR video poses similar problems because the perceived depth can vary greatly. In stereoscopic 3D applications, depth conflicts affect small on-screen widgets such as mouse cursors [95] or gaming crosshairs [96], although game designers can utilize known depth information from the 3D scene to minimize conflicts [96, 97]. In contrast, this 
dissertation focuses on handling depth conflicts in a dynamic VR environment, where the arrangement between the UI and the video is unpredictable.

Commercial video viewers for stereoscopic VR video do not handle depth conflicts directly. For example, the GoPro VR Player does not display UI elements during playback, and the Oculus Video application renders the video image as monoscopic when UI elements are shown on top. JauntVR's player renders timeline widgets and subtitles close to the viewer.

Dynamic stereo adjustment methods help reduce visual discomfort in stereoscopic scenes, an idea first proposed by Ware et al. [114]. These methods adjust the focus plane such that it aligns with the object the viewer is looking at, which can help reduce VAC and enhance depth perception in 3D scenes [85, 61]. The dynamic depth technique introduced in Chapter 5 also adjusts the depth of the UI widgets dynamically. The novelty lies in tuning the adjustment based on the perceived depth of the video to reduce depth conflicts. 


\section{Vremiere: In-Headset VR Video Editing}

Filmmakers are creating Virtual Reality (VR) experiences today by capturing VR videos, but video editing is still done primarily in traditional 2D desktop GUI applications such as Adobe Premiere. These interfaces provide limited capabilities for previewing content in a VR headset or for directly manipulating the $360^{\circ}$ video in an intuitive way. As a result, editors must alternate between editing on the desktop and previewing in the headset, which is tedious and interrupts the creative process.

This chapter demonstrates an application that enables a user to directly edit $360^{\circ}$ video while fully immersed in a VR headset. We first interviewed professional VR filmmakers to understand current practice and derived a suitable workflow for in-headset VR video editing. We then developed a prototype system implementing this new workflow. Our system is built upon a familiar timeline design, but is enhanced with custom widgets to enable intuitive editing of $360^{\circ}$ video inside the headset. We conducted an expert review study and found that with our prototype, experts were able to edit videos entirely within the headset. Experts also found our interface and widgets useful, providing intuitive controls for their editing needs.

\subsection{Motivation}

Virtual Reality (VR) video is emerging as a new medium for shared, creative experiences. VR video specifically means spherical panorama (full or partial) video viewed within a head-mounted display. Filmmakers use multi-camera rigs to capture $360^{\circ}$ videos and then edit them with standard video production software such as Adobe Premiere or Apple Final 


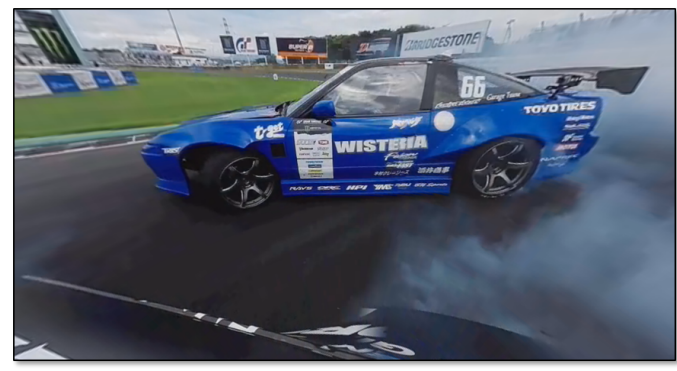

(a) View in the VR headset

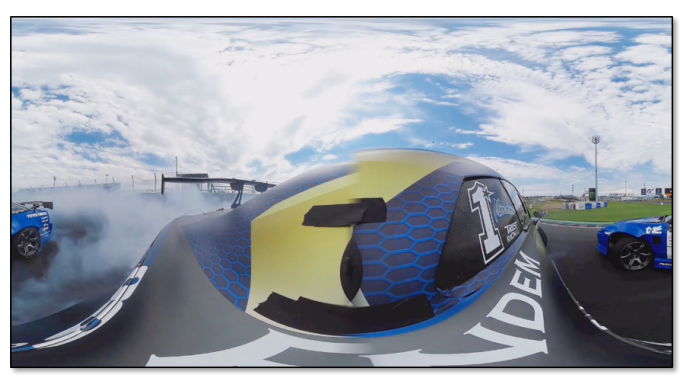

(b) View on desktop

Figure 3.1: The same shot, viewed in a headset versus on the screen. VR views look very different than on the desktop screen, and provide a much stronger sense of immersion. (C) TOYO TIRES JAPAN

\section{Cut Pro.}

However, these tools only support editing VR video in its flattened equirectangular projection format (Figure 3.1). One prominent director describes her first experience with editing $360^{\circ}$ video:

The first assembly went as well as expected. Rough in parts. A few nice visual match-cuts. Music with layers that gently guided the visuals. Compositionally sound, wides to close-ups and back again. ...

Overall, I felt the edit had a lot of promise, and I was quite pleased with myself. And then I watched it in a headset.

I don't think I'll ever be able to truly describe how much the edit sucked in a headset... [17]

This anecdote illustrates a major problem of editing VR video: viewing VR video in a headset is a dramatically different experience from viewing it on a desktop display. This difference manifests in many ways. In VR, a viewer sees only a portion of the environment at any time and must decide where to turn their head to view; the filmmaker cannot assume the viewer will look in a particular direction at a given time. VR video is immersive and takes time to absorb, which affects the pacing of cuts. Conventional video edits such as 
zooming and jump cuts can be jarring and confusing in VR. Editors must consider the relative spherical rotation of adjacent cuts. They must also deal with the potential strain, fatigue, and nausea of VR both for the editor, and for the intended viewer. At present, VR editors must follow an onerous workflow, alternating between editing on the desktop and then putting on a headset to review changes. Being able to perform WYSIWYG editing directly in the headset—and having tools for editing tasks specific to VR—would significantly improve their process.

This chapter describes initial exploration, development, and evaluation of an in-headset VR video editing interface. We first interviewed a number of VR video professionals to understand the current practice and difficulties of editing VR video on the desktop. Based on these pilot interviews, we describe the major tasks in VR video editing and the design requirements of an editing tool to carry out these tasks.

Based on our design requirements, we developed a prototype system called Vremiere that allows users to edit a video entirely in the VR headset. Our system is built upon a timeline design commonly used in commercial systems, with mouse and keyboard as input. The timeline design provides familiar context for trained video editors, enabling easy transfer of skills. We enhance our design with a collection of interface widgets to support manipulation and navigation of $360^{\circ}$ video while inside the VR headset. Specifically, our widgets enable 1) spherical visualization to aid navigation, 2) trimming and alignment of cuts, 3) placement of 2D images (titles) directly on the video, 4) annotating with bookmarks for review, and 5) adaptive vignetting to reduce fatigue.

Finally, we present the results of an expert user evaluation conducted among video editors with significant VR video experience. Experts were asked to use our system in a freeform video editing tasks, and provided us with feedback on both the system and the new workflow. 


\subsection{VR Professional Interviews}

We interviewed four professional VR video editors to understand VR video and how they currently edit. These professionals include a VR editor/filmmaker, a VR technical art lead, a creative director of a VR studio, and a VR video editor. Since VR video editing is still fairly unexplored, this broad set of discussions allowed us to understand current practice, identify limitations, and also find design requirements for our system from multiple perspectives.

Video editors currently follow the traditional video workflow to produce VR video. As described by Freeman et al. [41], this workflow includes three stages: pre-production (planning), production (capturing), and post-production (navigating and sorting footage, editing, compositing). $360^{\circ}$ video capture has recently received significant interest from the camera industry, leading to cheaper and more robust cameras. However, editors face unique challenges in the post-production stage, since the available tools are not designed for $360^{\circ}$ video.

VR editors' difficulties with current workflows can be summarized by two main themes. First, because $360^{\circ}$ video is best experienced in the VR headset, editors must repeatedly switch between editing on the desktop and reviewing changes in the headset. Second, VR video editing creates new interface needs (such as spherical rotation) that are not handled well by current professional tools. As a result, editors resort to ad hoc approaches to editing $360^{\circ}$ video on the desktop. According to the model of Hutchins et al. [52], the gulfs of execution (editing $360^{\circ}$ video using $2 \mathrm{D}$ tools) and evaluation (breaking from the desktop to preview in the VR headset) are significant problems with this workflow, and there is a clear benefit to more streamlined and direct editing of VR video.

We assembled the interview feedback into a common set of workflow issues, which we detail now. 


\subsubsection{Navigating footage}

Editors frequently browse footage to find key moments and to review the available shots. This task is particularly important for VR video since the spherical environment offers alternative ways to tell a story within a single video. Navigating $360^{\circ}$ video in the headset, however, is currently difficult because of the absence of video control interfaces such as the timeline. One editor mentioned difficulty understanding the full $360^{\circ}$ scene in the headset, since only a single view is visible at a time. She also noted that choppy raw footage can cause nausea [57], forcing her to remove the headset.

\subsubsection{Assembling cuts}

Professional editors currently try to rotate the scenes across a jump cut to align interesting points in two clips [16]. This technique is often done to make cuts less jarring to watch in VR. Otherwise the viewer may get thrown off, or start to wander around and lose the narrative planned by the editor. One editor explained that in order to align effectively, she needs to view the video clips inside the headset and take notes. She will then look for the corresponding scenes on the desktop and use Premiere's offset tool to align the video. This process is even more tedious when there are more clips in the project, or when she wants to change the offset of an earlier clip and has to propagate the edit to all subsequent clips.

\subsubsection{Previewing edits}

All editors noted that being able to preview the changes they made in the headset during editing is a very important feature but is not well supported. Because the $360^{\circ}$ video is shown in desktop tools as a flattened projection, any adjustment to the video such as trimming, color grading, or placement of text can look very different when viewed in the VR headset. 


\subsubsection{Titling}

To add titles, one editor uses an external image editing application to pre-distort the title image so that it looks right in the equirectangular projection. To make any changes, he repeats the entire process. He also said that the title usually looks much smaller on the desktop compared to viewing it in the headset. The editor explained that the process of adding titles requires him to repeatedly export his project to view the video with the title in a Google Cardboard headset.

\subsubsection{Review and annotation}

One editor explained that she reviews all raw footage after capture, and then reviews the entire final video after editing. During reviews, she watches carefully and takes notes. Since she is wearing the headset during review, and current viewers do not support annotation, she writes blindly on a piece of paper while wearing the headset.

$360^{\circ}$ video shows the content in all directions, so she checks to make sure that the final edit does not contain inappropriate action that might have been missed during editing. This requires watching all footage at least twice, once each in opposite view directions.

\subsection{In-Headset Editing Workflow}

We propose a new workflow to address the above limitations by allowing a user to edit and preview directly in the headset. Our workflow integrates editing and previewing in a single experience, creating a direct manipulation interface that bridges the gulfs of execution and evaluation [52]. Furthermore, it enables WYSIWYG editing of $360^{\circ}$ video, since the user is viewing the video in its final output form.

One strategy we did not employ is to implement all steps of the editing process in the headset. There are tasks that we believe are easier to perform on the desktop, such as managing files and performing initial assembly of clips. Current VR displays are much 
lower resolution than desktop monitors and are not well-suited for complex interfaces. Furthermore, long-term usage of VR headsets is not well-studied and may cause problems such as fatigue and nausea. There may be other limitations as well, such as the user's inability to perceive their surroundings in a work environment.

Instead, our in-headset editing workflow is complementary to desktop editing. We envision editors working with a conventional desktop interface but performing certain steps of the process in a headset. The editor may decide which tasks to do in headset versus on the desktop. One advantage of this approach is that we can reduce the complexity of the headset interface. We do not need to show every feature to the editor, only those that are most important to use in the headset.

Based on our professional interviews, we identified the following tasks as most important for in-headset interaction: timeline editing, video browsing, rotation alignment, titling, and bookmarking and reviewing. Some more advanced tasks such as spatial audio authoring or color correction are also interesting to explore in future work.

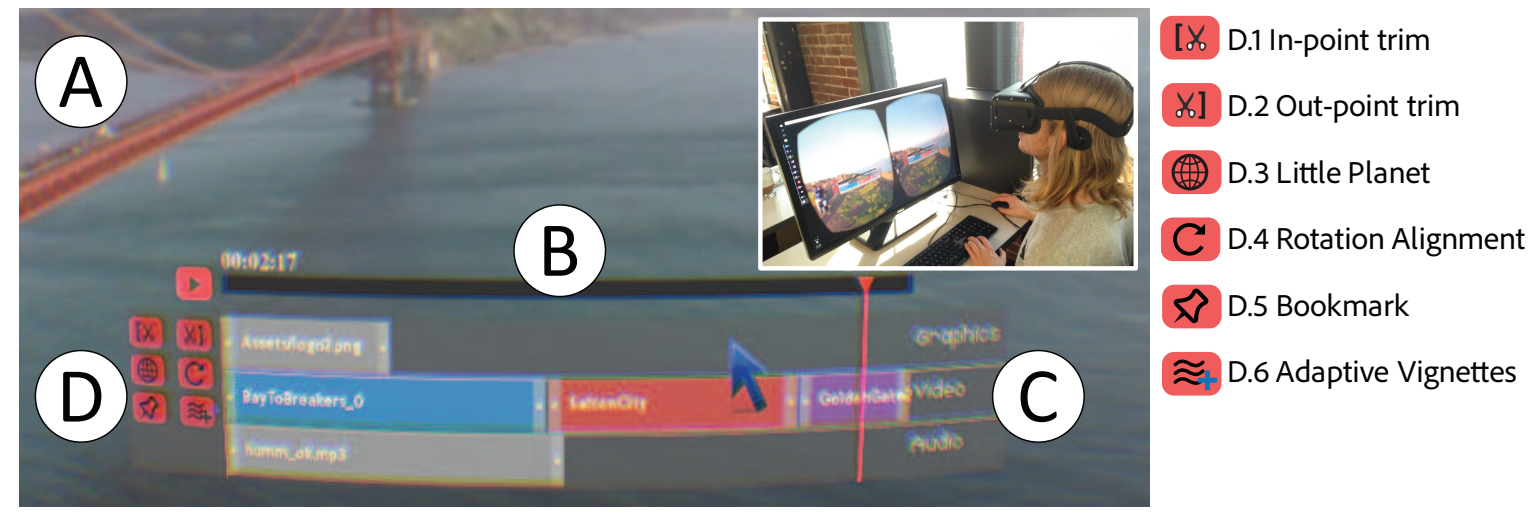

Figure 3.2: The Vremiere interface contains a video view in the background (A) and floating UI components such as the timeline (B), editing tracks (C) and editing widgets (D). By using familiar mouse and keyboard interaction, an editor can edit and view the $360^{\circ}$ video directly inside the VR headset.

Throughout our workflow, we emphasize WYSIWYG direct manipulation. We use the timeline metaphor that users are already familiar with from desktop software. Our interface is simplified, containing only the controls that are relevant for our in-h eadset editing tasks. 
We also provide tools to make the process more comfortable for the editor, including tools to avoid nausea [54] when navigating shaky video, and techniques to allow $360^{\circ}$ navigation without requiring constant head rotations.

For input devices, we rely on the mouse and keyboard. Our interface is orthogonal to the choice of input device. Expert professional editors typically prefer fast interaction over exotic input techniques, often memorizing most keyboard shortcuts in order to be able to work more quickly. Sitting on a swivel chair with a wireless keyboard and mouse, an editor can have a relatively comfortable and unencumbered editing experience.

\subsection{The Vremiere System}

We developed Vremiere, a prototype in-headset VR video editing system. We enable users to perform the target editing tasks while fully immersed in the VR headset. We focus on implementing a complete in-headset workflow and leave desktop software integration as future work. Vremiere runs on the Oculus Rift CV1 headset with rotational tracking, on a Windows 10 desktop with a GeForce GTX 970 graphics card. Our system uses the $80^{\circ}$ horizontal field of view of the headset. Currently Vremiere only supports monoscopic video.

Figure 3.2 presents the main timeline interface for our system, which is overlaid on a $360^{\circ}$ video view. This setup allows the user to quickly access editing and navigation tools simultaneously during the playback of the video or reviewing and browsing it.

Tools are accessible via a palette of buttons to the left of the timeline. Video browsing is enhanced by the Little Planet visualization (Figure 3.2D.3), and discomfort reduced by Adaptive Vignettes (Figure 3.2D.6). Jump cut alignment is visualized and adjusted using the Rotation Alignment tool (Figure 3.2D.4). Titles and 2D images can be positioned directly on the video (Figure 3.2 C "Graphics" track), and bookmarks can be placed on the video as well (Figure 3.2D.5).

In the rest of this section, we describe these features in detail. 


\subsubsection{Timeline editing}

The editing interface contains the minimal set of conventional interface elements needed to support our target in-headset editing navigation and editing tasks (Figure 3.2B). It comprises a play/pause button and a timeline, including three main tracks for manipulating assets: graphics, video, and audio (Figure 3.2 C). The interface is view-stabilized, as is the mouse cursor, so everything is attached to the headset view and is always readily accessible. Interface elements are rendered at infinity (monoscopic viewing) and composited on top of the $360^{\circ}$ video.

The user can perform basic arrangement and trimming edits on the tracks. To arrange, each block can be dragged along its track. To trim, the user can either drag the left and right handles of each block, or set the in and out trim points using the buttons (Figure 3.2 D.1 and D.2). Hovering the cursor on top of a block will display its original duration. The interface can be re-positioned by dragging with the right mouse button.

\subsubsection{Video browsing - visualization}
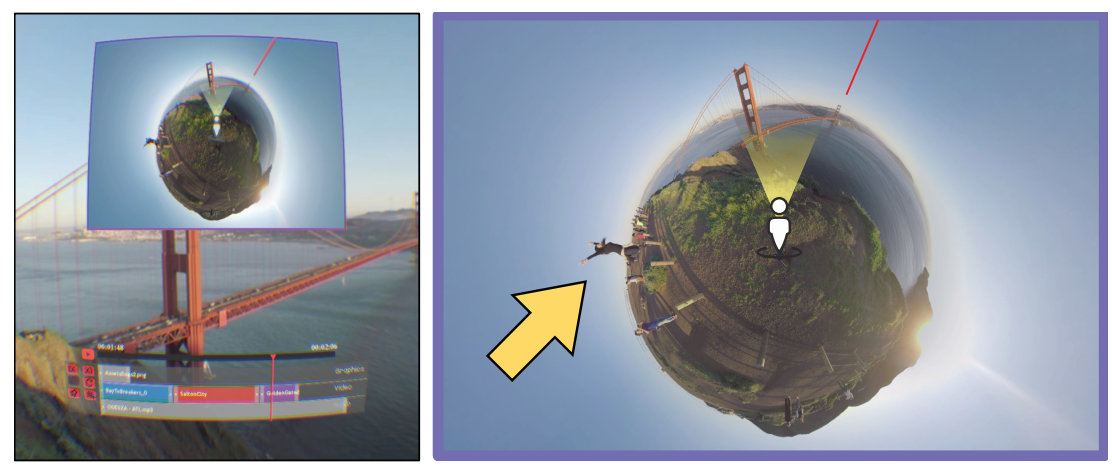

Figure 3.3: Left: the Little Planet is shown in a small window above the timeline to aid in-headset video navigation. Right: close-up view of the visualization; it can be used both as a minimap and a compass of the $360^{\circ}$ image. As noted by the yellow arrow, the user can quickly spot an interesting event outside her field of view (indicated by the yellow fan at the center) that would be otherwise difficult to find.

Video browsing is typically defined as interactive exploration to search for specific 
content, or to find inspiration from video content [99]. Our timeline interface supports standard browsing interaction. However, it does not always work well in a VR headset, as we learned from the initial expert interviews.

During VR video viewing, the user sees only a portion of the scene. This can make it difficult to get a sense for all the events in a video, or to find a specific event in some direction. Vremiere allows users to rotate the scene by clicking and dragging on the video to adjust "true north" and make it more comfortable to view other parts of the scene, but still the whole scene cannot be viewed simultaneously.

In order to make browsing and search more effective, Vremiere includes a Little Planet visualization (Figure 3.3). This visualization is both a minimap and a compass of the scene. In Figure 3.3, the user quickly maps her current view in the video with the view in the visualization; she can also spot the jumping lady behind her that she might have not noticed before. The circular, clock-like shape of the visualization enables her to quickly orient herself to that event, or simply drag on the visualization to navigate there by rotating the scene. We also support zooming using the scroll wheel to reveal more details.

The Little Planet is a stereographic projection of a sphere to a plane that has been wellstudied in map projection research [62] or used in VR navigation [37]. While it becomes distorted along pitch, it was found to be useful in spatial search [81]. This projection preserves angular properties [62], which has two important implications. First, objects moving around the scene can be followed continuously. Second, Little Planet represents well the spherical characteristic of the video, allowing for better egocentric orientation. It has been reported that such a representation was favored by users when searching for targets along the yaw dimension of a spherical image [81]. Equirectangular projection has also been used to show overview in a desktop-based video player [82]. However, equirectangular projection is often not recommended when observing objects moving around the scene, or when judgement of egocentric directions is important [14].

Following the design of overview + detail applications [27], our system displays Little 


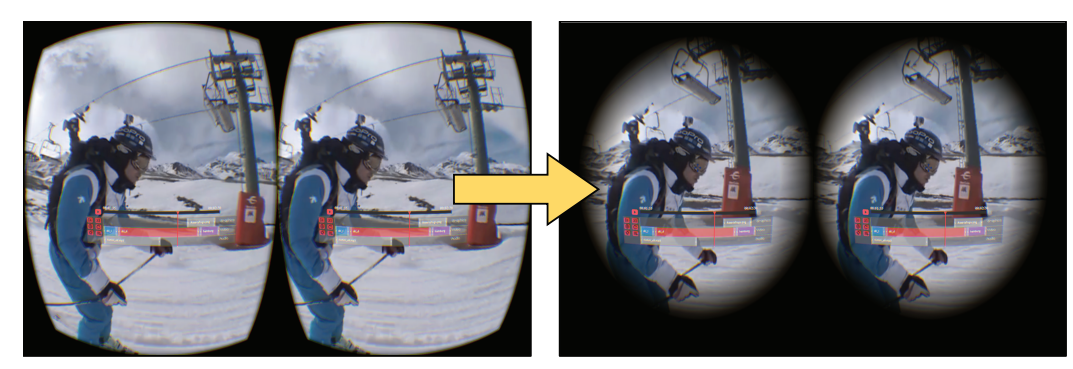

Figure 3.4: Our system dynamically contracts a pair of vignettes in front of the user's eyes based on the perceived motion in the video, to help reduce discomfort during video playback or scrubbing. Note, on the left, the default diameter of the vignette is $120^{\circ}$, which corresponds to no vignetting. (C) Ábaco Digital Zaragoza

Planet as a secondary window that shows the overview of the scene. This window is toggled by a toolbar button (Figure 3.2 D.3) or by pressing the Up key on the keyboard. This setup allows the editor to focus on the main content of the scene and trigger the visualization only when necessary. To help users quickly orient and navigate the space (when switching views), the visualization is rotated along yaw so that the up direction always maps to the user's current view. A yellow fan marker is added to help users visualize the field of view (FOV), and a red line marks the true north direction of the video.

\subsubsection{Video browsing - comfort}

VR can induce symptoms similar to motion sickness [39, 57], particularly for video with shaky or rotating scene motion. This disturbing motion is accentuated in video editing when the user scrubs along the timeline, speeding through the video. While shaky video can be stabilized using video stabilization techniques [54, 58], editors will often need to work with raw footage in order to save time and avoid distortions introduced by stabilization, and even smooth video may still be jumpy when scrubbing.

To make video browsing more comfortable, we provide an adaptive vignetting option, inspired by the method of Fernandes and Feiner [39], who restricted the user's FOV dynamically, based on gamepad inputs. We use the same vignette setup of [39] $]^{1}$, but our system

\footnotetext{
${ }^{1}$ please refer to Fernandes and Feiner [39] for more details on the design of vignettes
} 
dynamically contracts the vignettes based on the perceived motion of the video from the user's current viewpoint (Figure 3.4). When the user is watching in the headset, either normally or through scrubbing the timeline, the vignettes contract faster in shaky scenes and slower in steady scenes. The vignettes' diameter is measured in the diagonal field of view of the headset. Their default diameter is $120^{\circ}$, which corresponds to no vignetting. They stop contracting at $60^{\circ}$ to avoid blocking too much of the view, and expand back to the default size when the user pauses the video or stops scrubbing.

To compute the perceived motion, we use optical flow as a proxy to estimate how shaky the video scene is. This approach is similar to the system by Pongnumkul et al. [91], which also used the number of detected image features to approximate the visual quality of video scenes. We precompute optical flow for all videos using the Lucas-Kanade method [72]. During operation, Vremiere computes the motion magnitude of the user's current viewpoint in the video and uses it to determine the contraction rate of the vignettes. We followed the approach of Cheng et al. [22] to define the motion magnitude for the user's current viewpoint as:

$$
M_{f}=\sum_{i=1}^{N}\left\|V_{i}\right\|
$$

where $N$ is the number of tracked points in the user's current view and $V_{i}$ is the motion vector from the current frame $f$ to the next frame $f+1$ of point $i$.

When the video plays, the contraction rate is set proportionally to a value between 0 and $-30 \%$ based on $M_{f}$. These values were defined empirically to make the vignettes contract fast enough in choppy scenes but still subtle in normal scenes. The rate is set to 0 when $M_{f} \approx \min \left(M_{f}\right)$ and to -30 when $M_{f} \approx \max \left(M_{f}\right)$, respectively. $\min \left(M_{f}\right)$ and $\max \left(M_{f}\right)$ are precomputed over all $M_{f}$ of which viewpoints are centered around all tracked points in all the video, before launching the application for the video clips the user is editing. When the user scrubs the video, our system speeds up the contraction rate by multiplying it with 

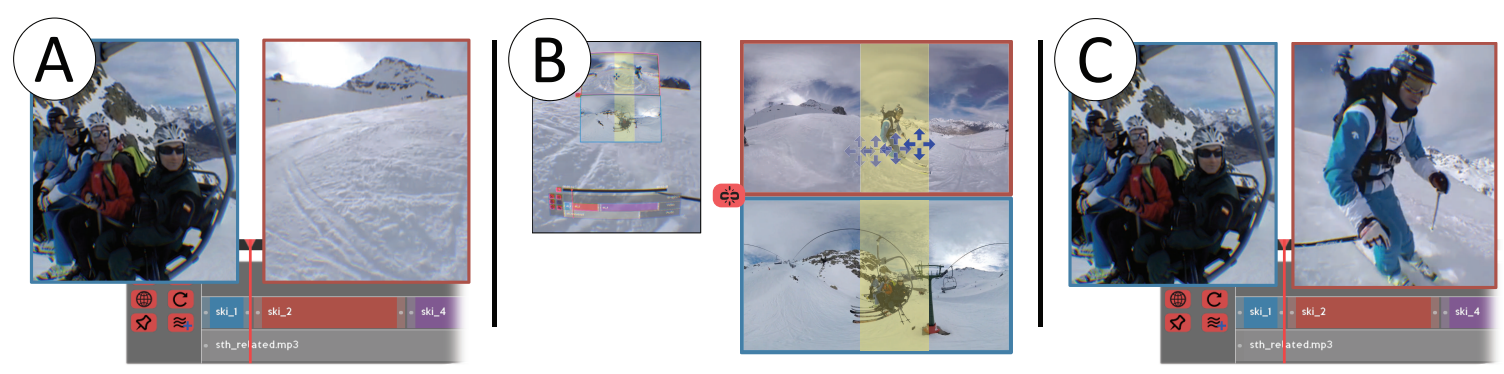

Figure 3.5: A: an example of a jarring cut in VR video. The cut transitions from the ski lift to an empty view of the mountain in the ski_2 video. B: Our Rotation Alignment tool is shown above the timeline. In the close-up view, the user can visualize how the cut transitions from one clip to another given a specific viewpoint in the video (visualized by yellow bars); she can also directly drag on the clips to align events before and after a cut, thereby helping the viewer to follow key elements in the video. Here, the Ripple Rotation mode is shown currently as an Unlink toggle. C: after rotation, the skier in clip ski_2 is aligned to the previous shot, resulting in a much better cut. @ Ábaco Digital Zaragoza

the number of frames changed during scrubbing. Therefore, scrubbing the video faster will contract the vignettes faster.

\subsubsection{Rotation alignment}

In our initial expert interviews, two editors stressed the importance of being able to rotate the video shots between cuts in order to align the interesting moments between them [16]. We use the term "rotation" loosely to mean offsetting the video pixels uniformly around the yaw axis. We also define a good cut in VR video as a horizontal (yaw) alignment of points of interest before and after a cut. While it is possible to align a $360^{\circ}$ video about roll and pitch as well, we focus on yaw because interesting content in $360^{\circ}$ video often happens along this dimension. Also, roll and pitch tend to be fixed for $360^{\circ}$ video to keep the horizon level.

Vremiere includes a dedicated tool called Rotation Alignment (Figure 3.5B). It shows an equirectangular projection of the clips before and after the cut and supports useful interaction to fine tune the alignment.

The visualization shows two vertically-stacked panoramas: the last frame before the cut, 
and the first frame after the cut. The equirectangular format was chosen instead of the Little Planet projection because it can better show the horizontal alignment, while still providing a good overview of the scenes. The user's horizontal FOV is visualized in each panorama as yellow vertical bars. These bars rotate with the user's viewpoint, so she can quickly see how the views before and after the cut are aligned.

We support several interactions to allow the editor to fine tune the alignment. First, the user can trim the video clips using the timeline and see the frames around the cut update in real-time on the panoramas. Second, hovering the mouse cursor over a panorama will update the main video view to that frame, allowing the user to quickly check the current alignment without having to adjust the timeline. Third, the user can rotate a video by clicking and dragging directly on its corresponding panorama. For example, in Figure $3.5 \mathrm{C}$, the user has aligned the ski lift before the cut to the skier after the clip, rather than transitioning from the ski lift to an empty view.

In standard video editing tools, rotations are defined with respect to an absolute orientation independently per-video clip. This can be cumbersome in large video projects, as editors generally care about relative orientation between shots. Adjusting the rotation of an early clip can break later cuts. We provide a mode called Ripple Rotation that propagates rotations to subsequent clips. Vremiere displays a Link/Unlink toggle in the rotation alignment tool to indicate the relationship between two clips (Figure $3.5 \mathrm{~B}$ ). Enabling this toggle will set the rotation of the video after the cut to be relative to that of the video before the cut. In Figure $3.5 \mathrm{~B}$, if the toggle is off, the user can change the rotation of each video individually; otherwise, rotating the blue (ski_1) video will also rotate the red video (ski_2) by the same amount.

We experimented with visualizing the rotation alignment by superimposing the frames before and after the cut and allowing the user to directly drag on the video to change its rotation. We discarded this design because our pilot testing revealed two limitations. First, blending two frames makes it harder to see the precise alignment. Second, dragging on 

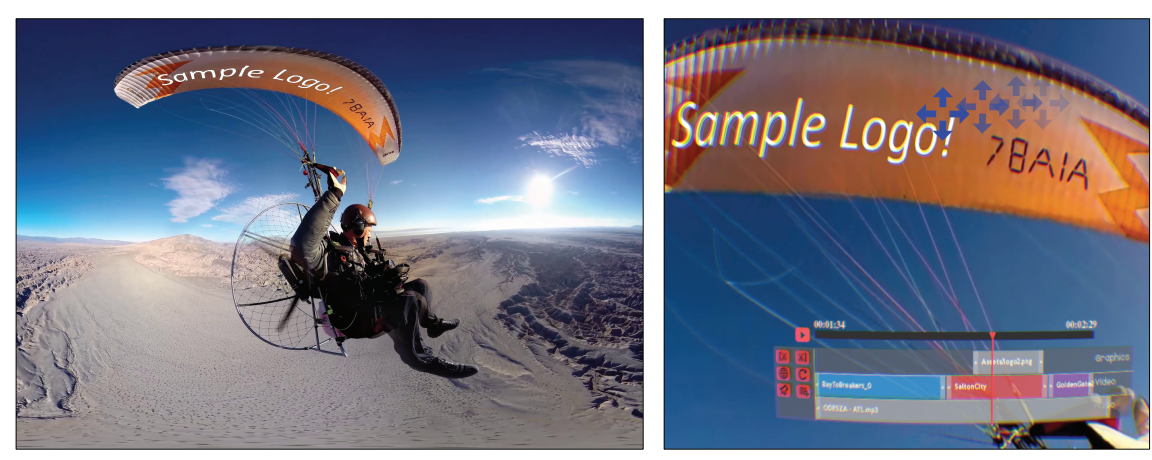

Figure 3.6: Left: On desktop, adding 2D text or images to a $360^{\circ}$ video is often unintuitive. Right: in the headset, our system provides a natural view of the image (Sample Logo) and enables users to directly place it anywhere in the scene. () P J Orravan

the video induced discomfort in some pilot testers. Previous research in simulator sickness advises against rotating the scene in front of the user [57].

\subsubsection{Titling}

As discussed in our expert interviews, editors often need to place 2D images of text or logos in VR video. Overlaying 2D images on a video is a basic form of video compositing and is an important operation in making VR videos. On the desktop, editors can use plug-ins to convert 2D images into equirectangular projection before applying it to the video, but the distorted form of the image makes editing and placing it unintuitive. In particular, it is very difficult to tell how big objects will appear in the headset (Figure 3.6 left).

Vremiere allows the editor to load and view a 2D image in the headset and provides WYSIWYG controls to place it anywhere in the VR video. The image is projected onto the view sphere at infinity (monoscopic viewing), and appears undistorted (straight lines are preserved) when placed at any position in the scene and viewed from any angle. The user can click and drag on the image, which is selected by casting a ray through the $2 \mathrm{D}$ mouse position to the view sphere and intersecting it with the image. Because the cursor is view-stabilized, the image position also becomes view-stabilized while dragging. To click on the image when it is not in the current view, the user can locate it quickly using the Little 


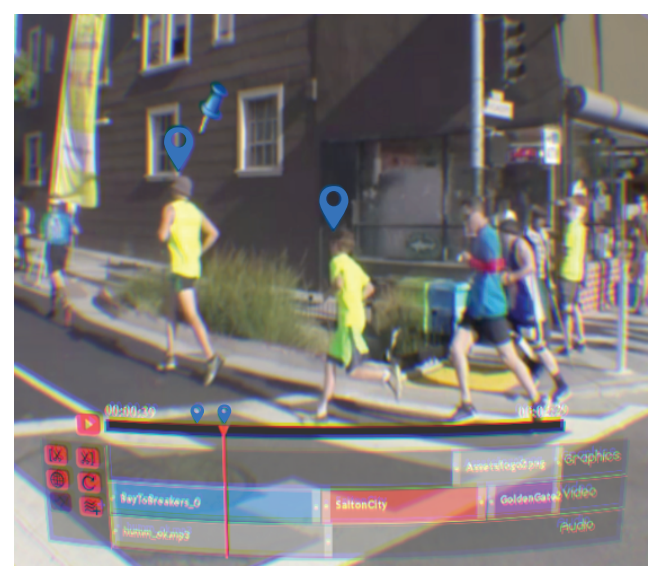

Figure 3.7: The user can directly click on the video to add markers. These markers are visualized on the timeline and can be reviewed quickly using keyboard shortcuts. (C) Jacob Phillips

Planet tool discussed earlier. For example, in Figure 3.6, the editor can easily position the logo image on the parachute (Figure 3.6 right), which is difficult on the desktop due to distortion (Figure 3.6 left).

\subsubsection{Bookmarking and reviewing}

Video editors use timeline markers for a variety of purposes, including synchronizing with music, reviewing footage, and giving feedback. Wearing a headset makes it difficult to take notes or speak directly with a colleague, so timeline markers are even more important.

Standard marker tools are inadequate for VR video. Because the video content shows in $360^{\circ}$, a marker needs to be placed on the video both at a specific time and at a specific location in the scene. The spatial location of markers presents a challenge when the editor needs to review them in the headset, because in order to find a marked scene element, the video must be adjusted to the bookmark's timestamp, and the view must be adjusted to point to the bookmark's location.

Vremiere allows users to add markers to a video and quickly review them in the headset. When the user activates the Bookmark tool (Figure 3.7, Figure 3.2D.5), the cursor changes to a pin icon. The user can click directly on the video to place a marker. Our system casts 


\begin{tabular}{l|c|l|l|} 
Participant & VR video experience & \multicolumn{1}{c|}{ Target platform } \\
\hline P1 & 1 year & Desktop, Mobile VR & Adobe After Effects \\
\hline P2 & 0.5 years & Samsung Gear & Autopano, Adobe Premiere \\
\hline P3 & 1.5 years & Oculus Rift DK2, HTC Vive & Autopano, Final Cut Pro, Ricoh Theta software \\
\hline P4 & 2.5 years & HTC Vive, Google Cardboard & Adobe Premiere, Ricoh Theta software \\
\hline P5 & 1 year & Google Cardboard & Adobe Premiere \\
\hline P6 & 1 year & Google Cardboard & Autopano, Adobe Premiere \\
\hline
\end{tabular}

Table 3.1: Participants' demographics

a ray from the camera center following the cursor direction and places the marker at the intersection between the ray and the video sphere. The markers are shown as pins on both the video and the timeline. The user can browse to each marker by pressing the left or right keys on the keyboard. When a marker is selected, Vremiere navigates to the marker's time and centers the view on the marker. The shift to another marker updates the view instantly, allowing users to browse notes quickly while creating minimal camera motion that can cause disorientation [57]. Alternately, we could show visual indicators on the headset view to help editors search for markers. While indicators are less disrupting, it might be slow when the editor wants to browse through notes quickly.

\subsection{User Study}

We conducted an expert review to evaluate how our system can support VR video editing. Specifically, we wanted to see 1) if our in-headset editing workflow adds significant value to VR video editing and 2) how our editing interface and widgets aid users in editing $360^{\circ}$ video in the headset.

A direct comparison of our system with a professional editing suite would be difficult. Since there are currently no standard tools to edit VR video, editors often have different ad hoc workflows or use third-party plug-ins. Thus, there is no clear baseline for comparison. Alternately, we could use a desktop version of our interface as a baseline. While this comparison would allow us to study the benefit of in-headset editing, it would not measure the utility of our widgets, which were designed specifically for in-headset video interaction. 
Therefore, we focus on a qualitative evaluation via expert review on a freeform video editing task to gain insight from experienced users about both our new workflow and our system. We collected feedback on workflow, utility, and usability of Vremiere, as well as subjective preference between Vremiere and the expert's own workflow. We also measured pre- and post- Simulator Sickness Questionnaire (SSQ) scores [55] to observe the comfort level of participants.

We recruited six professional video editors ( 1 female), aged 19 to 41 (Table 3.1). The participants have significant experience with VR video production, having either published a VR video or been a part of a production team that produced a VR video. One editor participated in our inital interviews but was not involved in the design of Vremiere. Each participant was compensated with a $\$ 25$ gift card for their time (approximately one hour).

\subsubsection{Procedure}

\section{Introduction and training}

Upon arriving at the study site, participants were asked about demographics and then completed a pre-exposure SSQ. They were then introduced to the system. An experimenter demonstrated each feature in the system and explained how it can be used to edit video in the headset. Participants were asked to put on the headset and tried all the features on a test video.

\section{Main task: freeform video editing}

Participants were given four $360^{\circ}$ video clips (total time $\approx 3.5$ minutes), an mp3 soundtrack, and a logo image, and were asked to produce a VR video for the Oculus Rift headset. The clips show scenes of various cities in the world that were suitable for a travelogue video. Before the task, participants were told to take as much time as needed and were encouraged to continue the task until satisfied. 


\section{Questionnaire and interview}

After the task, participants completed a post-exposure SSQ questionnaire, and another questionnaire that consists of four 7-point Likert scale questions about various aspects of our workflow and system. Finally, participants were interviewed with open-ended questions about the workflow and specific parts of the system.

\subsubsection{Results}

Reported results follow a 7-point Likert scale format, with 1 labelled "strongly disagree" and 7 labelled "strongly agree."

\section{In-headset editing workflow}

All participants completed the task in the VR headset and reported they were satisfied with their result. Participants responded overwhelmingly that our in-headset editing system would be very useful for editing VR video. When asked to rate their agreement to the statement: "My workflow through the entire interface was coherent and fluid", one rated 7/7, three rated $6 / 7$, and two rated 5/7. Comments from the interviews point to several benefits of in-headset editing: able to edit and see the changes in real-time (P3, P5, P6), able to adjust fine details (P4), intuitive (P1, P2, P3), and save time (P6).

\section{Comfort level}

We were also interested in observing participant's comfort level during the study. Participants spent on average 16.12 minutes to finish the task (STD = 5.26). The SSQ score after the study $(\mathrm{M}=3.5, \mathrm{STD}=4.03)$ rises slightly compared to the score before the study $(\mathrm{M}=1.5$, $\mathrm{STD}=1.37$ ); the difference was not statistically significant (paired-samples t-test, $\mathrm{t}(5)=$ $1.309, \mathrm{p}=0.24)$. This indicates that participants were mostly comfortable throughout the study. While our system provides the adaptive vignettes option, only one participant used it. 


\begin{tabular}{|l|l|l|l|l|l|l|l|}
\cline { 2 - 7 } \multicolumn{1}{c|}{} & \multicolumn{2}{c|}{ D } & N & \multicolumn{3}{c|}{ A } \\
\hline \multicolumn{1}{c|}{ Edit operations } & $\mathbf{1}$ & $\mathbf{2}$ & $\mathbf{3}$ & $\mathbf{4}$ & $\mathbf{5}$ & $\mathbf{6}$ & $\mathbf{7}$ \\
\hline $\begin{array}{l}\text { Preview the video directly } \\
\text { in the headset }\end{array}$ & & & & & & & 6 \\
\hline $\begin{array}{l}\text { Timeline navigation } \\
\text { of the video }\end{array}$ & & & & & 1 & 1 & 4 \\
\hline Trimming & & & & & 1 & 3 & 2 \\
\hline Rotation alignment & & & & & 1 & 1 & 4 \\
\hline Titling & & & & & & & 6 \\
\hline Bookmark and review & & & & & & 1 & 5 \\
\hline
\end{tabular}

Table 3.2: Summary of responses to the question "I feel this editing operation is intuitive and easy to learn" (D: disagree, $\mathrm{N}$ : neutral, A: agree).

However, this participant spent the most time in the headset (23.07 minutes) and reported his eyes were more relaxed after the study. While this is promising, we attribute this result mostly to expert's familiarity with viewing video in VR.

\section{Interacting with $360^{\circ}$ video in the headset}

All participants rated their agreement with the statement "I feel this editing operation is intuitive and easy to learn" (Table 3.2). This indicates that participants could easily edit $360^{\circ}$ video in the headset using our interface and widgets.

We also asked participants to rate the usefulness of each widget (Table 3.3). Overall, rotation alignment, titling, and adaptive vignettes received high ratings by participants. Meanwhile, Little Planet and bookmark and review received different opinions. Interview comments show that the usage of them depends on participants' personal preference and editing method. For Little Planet, participants who favored this tool used it very frequently throughout the task, based on our observation. They mainly used it to skim the footage before editing, or to find interesting moments to rotate and align the cut; one expert even used it to review the final edit. However, participants that did not find much use of Little Planet reported that they preferred to look at the video directly. For bookmark and review, we also found the utility of this tool depends on the expert's own experience with markers. 
Participants who favored this tool frequently used markers in their own workflow, and ended up using this tool more in our study.

\begin{tabular}{|l|c|c|c|c|c|c|c|}
\cline { 2 - 8 } \multicolumn{1}{c|}{} & \multicolumn{3}{c|}{ D } & N & \multicolumn{3}{c|}{ A } \\
\hline Vremiere Widgets & $\mathbf{1}$ & $\mathbf{2}$ & $\mathbf{3}$ & $\mathbf{4}$ & $\mathbf{5}$ & $\mathbf{6}$ & $\mathbf{7}$ \\
\hline Little planet & & 2 & & 1 & & & 3 \\
\hline Rotation alignment & & & & & 1 & 1 & 4 \\
\hline Titling & & & & & & 2 & 4 \\
\hline Bookmark and review & 1 & & 2 & & 1 & 1 & 1 \\
\hline Adaptive vignettes & & 1 & & & 1 & 3 & 1 \\
\hline
\end{tabular}

Table 3.3: Summary of responses to the question "I feel this widget is useful for my editing task in $V R "$ (D: disagree, N: neutral, A: agree).

\section{Subjective preference toward expert's own workflow}

When asked to compare each editing operation supported in our system with the expert's own desktop workflow, high ratings were found for certain tasks (Table 3.4). Specifically, previewing, rotation alignment, and titling were strongly favoured for in-headset editing. One participant described his titling process in Adobe Premiere including extraneous steps such as highlighting the video with a box that simulates the headset view and carefully checking the title to make sure it fits within that box. Any adjustment to the title was very tedious and unintuitive compared to using our system. Timeline navigation and trimming were also favored, but participants wanted more advanced timeline manipulation and trimming options as in a professional tool. Finally, participants were mostly neutral about the bookmark and review operation. Although all participants agreed it is a useful tool for reviewing the video, only one participant currently needs this tool to review footage and communicate with his team. 


\begin{tabular}{|l|c|c|c|c|c|c|c|}
\cline { 2 - 8 } \multicolumn{1}{c|}{} & \multicolumn{3}{c|}{ D } & N & \multicolumn{3}{c|}{ A } \\
\hline \multicolumn{1}{c|}{ Edit operations } & $\mathbf{1}$ & $\mathbf{2}$ & $\mathbf{3}$ & $\mathbf{4}$ & $\mathbf{5}$ & $\mathbf{6}$ & $\mathbf{7}$ \\
\hline $\begin{array}{l}\text { Preview the video directly } \\
\text { in the headset }\end{array}$ & & & & 1 & & 1 & 4 \\
\hline $\begin{array}{l}\text { Timeline navigation } \\
\text { of the video }\end{array}$ & 1 & & 1 & 1 & 2 & 1 \\
\hline Trimming & & 1 & 1 & & 3 & 1 & \\
\hline Rotation alignment & & & & & 2 & 2 & 2 \\
\hline Titling & 1 & & & 4 & & & 1 \\
\hline Bookmark and review & \multicolumn{2}{c|}{} & & & & & \\
\hline
\end{tabular}

Table 3.4: Summary of responses to the question "I prefer this editing operation in this VR system as compared to my current workflow". (D: disagree, N: neutral, A: agree)

\subsection{Discussion}

The results from the study demonstrate a clear preference from experts to be able to edit VR video in the headset. Despite the paradigm shift from the desktop to the headset environment and the lack of more advanced features often found in professional editing software, our workflow enabled experts to finish the task entirely in the VR headset. The most prominent benefit of in-headset editing is the ability to see changes and make adjustments directly in real-time. This is particularly important because video editing is a tedious task and editors require a tool that provides a fast feedback loop, so they can focus more on the creative task. As VR video is a recent medium and editors are still exploring new ways to create and tell stories through editing VR video, this finding motivates more explorations of in-headset techniques to support this new creative process.

Our system also provides essential tools needed for editing and interacting with $360^{\circ}$ video in the headset. At its core, our system is built upon familiar desktop editing interfaces and enhanced with new widgets to support in-headset interaction. It is clear from the study that this unique design allows expert editors to edit easily in the headset. More importantly, experts were able to use our new widgets to perform tedious tasks that are unique to VR video editing such as aligning cuts, placing $2 \mathrm{D}$ images in the video, or adding markers. 
While some of our widgets received different opinions from experts such as the Little Planet or the bookmark and review tools, they call for more in-depth exploration in techniques to support video navigation and collaboration in the headset.

\subsubsection{Requested features and limitations}

We collected feedback from participants on additional features that they want in the headset. All participants wanted more features in a professional suite such as advanced timeline, color grading, and keyframing, so they could get more editing done in the headset. A professional editing suite is a substantial piece of software implemented over years by teams of engineers. Our decision not to implement all features allows us to keep our in-headset interface manageable, but makes comparing our system with a professional suite difficult. Future work will need to explore more in-headset features and ways to evaluate in-headset editing with desktop solutions.

Some participants asked about the possibility of using more expressive controllers in Vremiere rather than mouse and keyboard. While our current setup is straightforward for expert editors, it still requires users to rely on their proprioception because they cannot see the input devices. In our study, none of the participants reported any problems, possibly because they had their hands on the keyboard and/or the mouse most of the time. Nevertheless, we believe using wireless input devices or an untethered headset could make the experience more comfortable. One interesting opportunity is to explore how these devices can support video editing in the headset. The traditional form-filling interaction style that involves heavy usage of mouse and keyboard is often used in creative tools for illustration, computer-aided design, or video editing. In video editing, some examples are tasks that require parameter adjustment or text-entry, which may not be desirable to do in the headset at the moment. While form-filling interaction is fast and precise on the desktop, it has been shown to be tedious and error-prone on other devices such as touch screen, and has been replaced in 
recent exploration with a more direct, object-oriented paradigm [115].

When asked what parts of VR video editing should be done on the desktop, participants wanted to do tedious organization tasks such as rough assembly or audio alignment before switching to VR entirely to fine tune the editing. Vremiere currently supports only basic desktop integration such as loading assets from the desktop and exporting the edited video. Meanwhile, a fully designed hybrid workflow that allows editor to switch between desktop and the VR headset merits more thorough investigation, similarly to research on hybrid 2D/3D design workflow [13].

Our system currently supports only monoscopic video. While Vremiere can also display stereoscopic content, some interface elements will need to be adjusted carefully to avoid creating contradicting depth cues with the objects in video. Existing guidelines from stereoscopic 3D game interfaces could be used to guide future stereoscopic support of our system [96].

\subsection{Conclusion}

We have presented Vremiere, an application that enables a user to directly edit $360^{\circ}$ video while fully immersed in a VR headset. Based on interviews with professional VR video editors, we explored and designed an in-headset editing workflow for $360^{\circ}$ video editing tasks that are difficult to do on the desktop. We then developed our system around this new workflow. Our system is based upon familiar desktop editing interfaces, but is enhanced with custom widgets to enable intuitive edting of $360^{\circ}$ video inside the headset. This unique design enabled editors to simultaneously view the $360^{\circ}$ video in its natural form, and to access tools to edit the video, resulting in a seamless editing workflow inside the VR headset. Our expert review study showed that our workflow and our system allow experts to edit VR video in the headset. Experts also found our interface and widgets useful, providing intuitive controls for their editing needs. These findings showed the potential of this new form of 
editing to support the growing creative needs around VR video. 


\section{CollaVR : In-Headset Collaborative Video Reviewing}

Chapter 3 introduced how various video editing tasks can be accomplished in the headset by individual editors. This chapter takes a step further and explore VR video authoring in the context of multiple users. In particular, this chapter focuses on collaborative video reviewing. Collaborative review and feedback is an important part of conventional filmmaking and now VR video production as well. However, conventional collaborative review practices do not easily translate to VR video because VR video is normally viewed in a headset, which makes it difficult to align gaze, share context, and take notes.

This chapter presents CollaVR, an application that enables multiple users to review a VR video together while wearing headsets. We interviewed VR video professionals to distill key considerations in reviewing VR video. Based on these insights, we developed a set of networked tools that enable filmmakers to collaborate and review video in real-time. We conducted a preliminary expert study to solicit feedback from VR video professionals about our system and assess their usage of the system with and without collaboration features.

\subsection{Motivation}

Collaboration and review are integral parts of the conventional filmmaking process. For instance, editors and directors frequently work in front of the same computer monitor, discussing edits while referring to the display. Likewise, in the "dailies" process, filmmakers gather to view individual shots on a large screen, discussing and giving feedback, while a coordinator controls playback. A producer or client may also review video on their own and then later give written notes to the editor or director. 

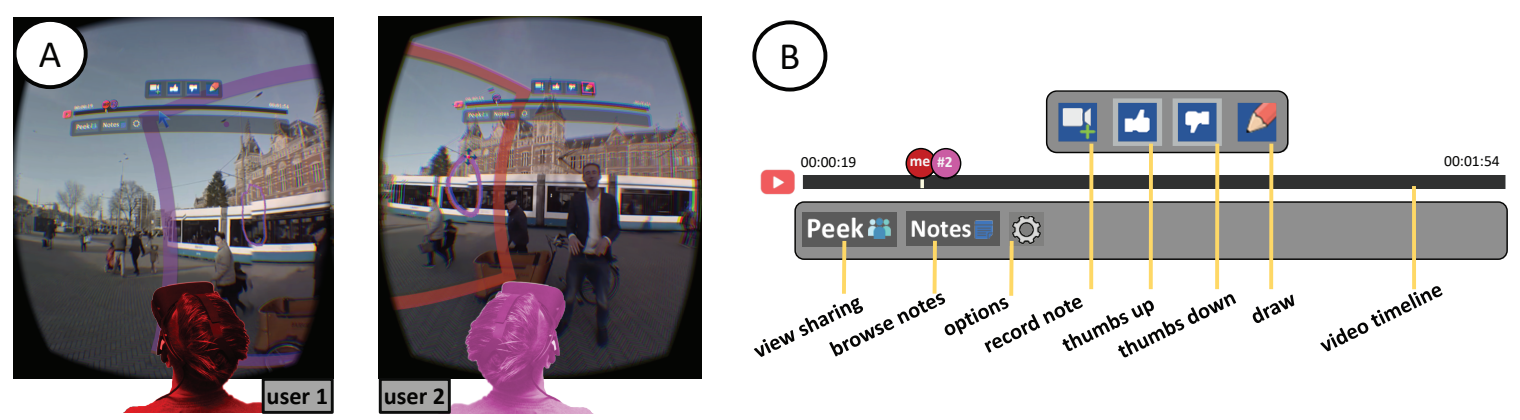

Figure 4.1: (A): In-headset views of two CollaVR users watching a video together. The clients are connected through a local network. In the headset, users see visualizations of each other's viewport. User 2 (purple) circles a stitching artifact on the video and the drawing immediately appears on user 1's (red) screen. (B): The timeline interface includes features to support communication, view sharing, and notetaking for VR video.

Virtual Reality (VR) video is an emerging art form with creative practices adapted from conventional filmmaking. However, collaborative review and feedback are much more difficult for VR video because fully experiencing VR video requires wearing a headset that blocks all view of the outside world. This interferes with almost every type of collaboration, because there are no affordances for awareness of others' actions. Two participants in the same room cannot easily point to a specific element in a video. Because each viewer effectively has their own video player, even synchronizing timing and view direction are difficult. The headset makes it difficult to use notetaking devices, such as paper or keyboard. In formative interviews with professionals, we found that editors suffer many of these issues and that current tools fail to create an effective shared environment for video reviewing.

We present CollaVR, an application that enables multiple users to review a VR video together while wearing headsets. We focus on supporting synchronous collaboration scenarios that are central to film production, in which collaborators can exchange feedback in-person or in dailies review sessions. Through formative interviews with professionals, we distilled several high-level goals for our system and developed a set of networked tools to achieve these goals (Figure 4.1). In particular, CollaVR's tools enable headset users to 1) quickly understand collaborators' activities and view directions in the headset to streamline 
discussion in the video, 2) share and synchronize video playback through interactions that resemble physical reviewing activities, and 3) record and review multimodal feedback directly in the headset.

We conducted a preliminary expert study to solicit feedback from VR video professionals about CollaVR. Our results show that experts are positive about the collaboration potentials of our system over a baseline interface that does not support collaboration. Our system allows experts to actively and collaboratively review video in the VR headset using natural interactions that are often seen in face-to-face collaboration. Groups of experts discussed video issues using more implicit than explicit verbal cues, spent more time viewing video together, and engaged in collaborative notetaking.

\subsection{VR Professional Interviews}

We interviewed professionals from four different VR video studios to inform our design. These professionals include a VR editor/filmmaker, a VR technical art lead, a VR director, and two VR editors. We asked participants to describe their current VR video reviewing workflow.

Video production is a collaboration among multiple stakeholders [87]. VR video's uniquely immersive nature requires that all participants review footage in-headset. For example, clients can ensure their brand message is recognizable, editors can find jarring scenes that may cause discomfort, and colorists can spot bad lighting. All the professionals we spoke with felt it was important to review footage in VR.

Unfortunately, our interviews also confirmed a pervasive issue with current reviewing practices: the benefits of face-to-face interaction are curtailed when collaborators wear VR headsets, and simple co-located reviewing tasks such as watching a video together or discussing an issue become very difficult. As a result, each studio has devised their own compromise review solution and there is no one standard "current workflow" in practice. 
We will now elaborate on the specific needs of the interviewees and how their workflows are adapted accordingly.

\subsubsection{Social awareness}

When reviewing a VR video together, editors need to understand where everyone is looking, both to confirm everyone saw important details in the video and to be able to have informed discussions. However, once someone is watching a video in-headset, there is no way to directly see what is in their current field of view. One editor mentioned that she frequently has to ask clients "did you see it?" Similarly, when the client gives feedback, the editor does not know what the client's viewing experience was, which inhibits understanding. High-end headsets (e.g., Oculus Rift, HTC Vive) can mirror the in-headset view on a desktop monitor, but video review frequently happens on mobile headsets (e.g., Google Cardboard, Samsung GearVR) which lack this capability. View mirroring is not currently supported remotely.

\subsubsection{Common context}

Video production involves people with different expertise and domain languages, making communication difficult. It can be even harder in VR video when everyone has to describe their experiences in an immersive environment. Thus, our interviewees stressed the importance of discussing and annotating the video together. One participant explained that when everybody is in a room together and looking at the same video, they can use pronouns like "this" and "that" (i.e., deictic references) and can gesture directly on the video to describe changes. With these natural interactions, an editor can discuss feedback directly with collaborators and there is ample opportunity to articulate or clarify comments. However, when users put on VR headsets, their activities are not shared and they must constantly ground the conversation by cumbersome techniques such as referring to timestamps or specific objects and events in the video (e.g., "at time 31 seconds, the person on the left in the blue shirt"). 


\subsubsection{View sharing}

We identified several activities that concern sharing or controlling the video view.

Looking over shoulder. One editor described an ad-hoc reviewing setup: each team member would load the VR video on a phone and watch separately by holding the phone in their hands and rotating it. Users could "peek" at other peoples' screens to gather information or exchange feedback while retaining control of their own video. However, the editor pointed out this method is only good for high-level feedback and coordination, because the video is not viewed in headsets.

Watching together. Several participants mentioned they frequently try to watch video simultaneously in their headsets. Watching together is a common technique in conventional workflows, where editors use a synchronization service such as Cinesync to control video playback [26]. Watching together in the headset allows each viewer to look around independently while synchronizing playback among peers. One editor explained that, when more people look at a video together, there are more opportunities to spot issues. However, controlling the video playback is difficult when each viewer has a separate player, particularly if viewers pause or rewind the playback. As a result, collaborators may not know if they are viewing the same events at the same time, making detailed discussion difficult.

Coordinated viewing. One participant described a "dailies" reviewing process in which the shot animator would select a predefined view direction and render a regular NFOV video. The conventional dailies process was then used for the rendered NFOV video: a coordinator would control the video playback and everyone else in the room would view the shot and discuss it. This shared viewing and control process does not currently exist in a headset and rendering to a regular NFOV video loses the VR viewing experience. 


\subsubsection{Notetaking}

Capturing feedback from a discussion is an important part of the review process. Editors often use review notes as a task list for the next day. Collaborators can share notes for asynchronous reviewing. Written notes, however, are hard to produce when wearing a VR headset. One participant shared that she writes notes blindly on paper. Moreover, VR video can require the reviewer to describe complex in-headset experiences with information about gaze direction, semantics, and visuals that may be difficult to capture in writing. Two editors mentioned that most notes they receive from clients are high-level and do not capture the nuances of the clients' feedback. Another editor said that notetaking during viewing is cumbersome because it requires pausing the video and interrupts the story's flow, so there is a disincentive to provide comments.

\subsection{Design Goals}

Our formative interviews make it clear that while shared experiences are a common part of traditional video collaboration, they are largely nonexistent for VR video review. As a first step to remedy this, we derived high-level design goals from the interview feedback:

1. Awareness. Enable natural social interactions such as observing gaze, listening to voice, and gesturing with hands.

2. View sharing. Establish a common context for discussion via view sharing techniques such as peeking, watching together, and coordinated viewing.

3. Notetaking. Enable recording and browsing feedback that captures the full VR video viewing experience.

For the case of synchronous, co-located review, we envision satisfying these design goals in a VR application user interface that allows several users wearing headsets to view a VR 
video, communicate with one another, and take and share notes. The VR interactions should resemble the natural, intuitive behaviors that are often used by co-located professionals in film studios for regular videos. These interactions could also generalize to remote or asynchronous collaboration, which we leave as future work.

\subsection{The CollaVR System}

Based on our design goals, we developed CollaVR, a system that connects headsets on a local network, allowing multiple users to review VR video together. Figure 4.1 shows an overview of CollaVR. We employ a client/server architecture. Each client is an instance of an in-headset VR video reviewing application that supports watching VR video and sharing feedback. The server is a separate process that connects clients; we normally run it in the background on one of the client computers. It receives and broadcasts state (e.g., current time and gaze direction) among all client systems and performs audio mixing for voice chat.

CollaVR currently supports the Oculus Rift CV1 and DK2 headsets with orientation tracking. Users interact with CollaVR using a standard desktop mouse and keyboard; handheld VR controllers can also be supported by emulating a mouse input. A microphone and headphones are required for voice chat. Since we focus on studio workflows where multiple people review video synchronously in-person, we assume our system is connected to a fast local network and multimedia files are stored locally on each client computer to reduce network bandwidth.

The client interface (Figure 4.1p) includes several tools and visualizations to facilitate collaborative video review. Specifically, our "awareness visualization" allows collaborators to quickly understand each other's status, our "view sharing" tools help people share views to establish common context for discussion, and our "feedback recording and browsing" tools allow users to capture and review multimodal notes. We will now detail each of the features of our client system. 


\subsubsection{Awareness visualization}

CollaVR employs a combination of visual and auditory cues to help users quickly understand what their collaborators are doing. In particular, we want to reproduce some of the benefits of face-to-face interactions that people often use in studio, such as observing gaze direction, listening to voice, and gesturing at the video. We convey this information through viewport visualization, spatialized voice chat, and activity visualization.
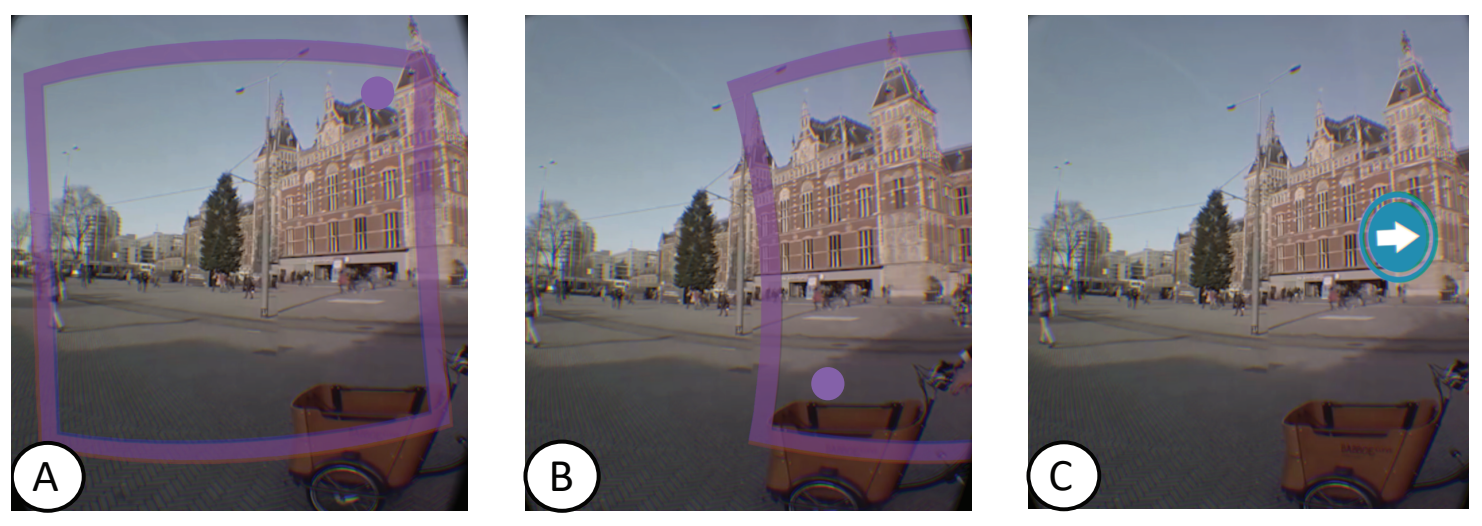

Figure 4.2: Left: Our system renders the viewport of a collaborator as a color-coded rectangle. The extent of the rectangle depicts the field of view. The 3D cursor pointer is also rendered inside the viewport, so users can point to a video scene element. Middle and Right: When the viewport travels outside the current field of view, an arrow appears on the periphery to provide a directional cue toward the off-screen position.

\section{Viewport visualization}

We visualize a collaborator's view as a rectangular viewport (Figure 4.2 a), rendered by projecting a rectangle centered on the collaborator's gaze onto the view sphere. The rectangle size matches the field of view of the collaborator's headset, so the user can understand what video elements are visible to the collaborator. The rectangle is rendered with a thick border, and is uniquely colored for each user.

When two users are discussing a video, if their gaze directions are not close enough, their fields of view may not overlap and the viewport visualization may be entirely offscreen, 
e.g. if the collaborator has turned too far to the right (Figure $4.2 \mathrm{p}$ ). In this case, we show an arrow icon in the periphery of the user's view (Figure 4.2 c) pointing in the direction of the collaborator's viewport. If the user turns to follow the arrow, when the collaborator's viewport is visible again the arrow goes away.

This visualization is only useful for collaborators viewing the same part of the video; in other cases, the visualization may just be distracting [44]. For this reason, we only enable this visualization when the collaborator is viewing video within seven seconds before or after the viewer, and the visualization can also be turned off in the settings as well.

\section{Spatialized voice chat}
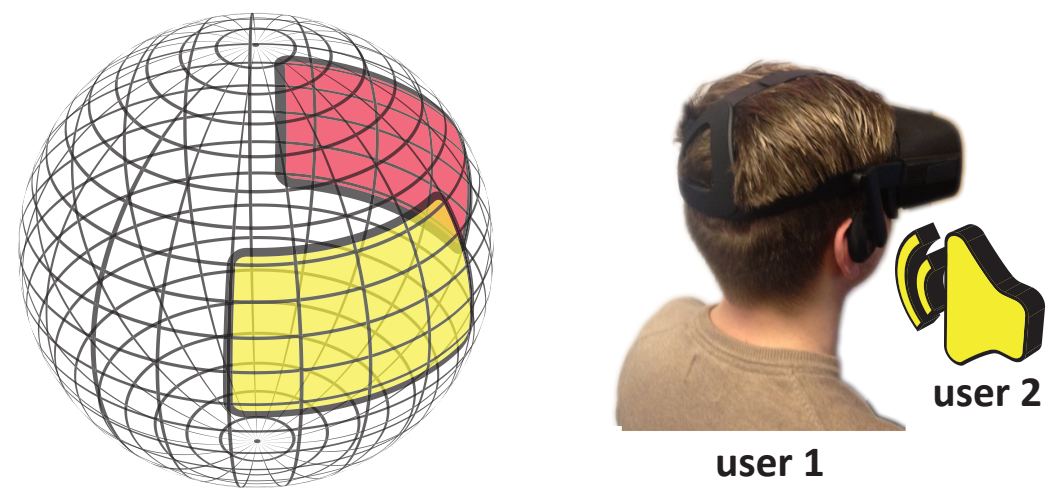

\section{user $1 \square$ user 2}

Figure 4.3: Illustration of spatialized voice chat between user 1 and user 2. As shown on the sphere, user 2 looks to the right of user 1. We spatialize user 2's audio stream so that user 1 will hear user 2's voice as coming from the right.

When immersed in VR with headset and headphones, even though users are co-located, they may not be able to hear one another clearly as they speak. Therefore, CollaVR supports voice chat by streaming audio from the headset microphone to all collaborators, mixed with the VR video's audio.

To further support spatial awareness of collaborators, we spatialize the microphone audio in $3 \mathrm{D}$ by making it seem as though a collaborator's audio is emanating from a position on the view sphere coincident with the collaborator's gaze direction (Figure 4.3). Spatial 
audio is perceptible even when the source is off-screen [44], and research has shown its effectiveness in aiding visual search [19] and video conferencing [7]. 3D spatial rendering is implemented in the server by transforming the user's single channel microphone audio stream using an off-the-shelf higher-order ambisonics audio library [100]. For each client, the server spatializes the audio streams of the other clients based on their view direction, mixes them to a single stereo audio stream, and sends the mixed audio to the client over the network. Sometimes the VR video sound can interfere with voice chat, so we let users mute the video's audio with a toggle in the "Option" panel.

Even when the viewport visualization is turned off to minimize visual distractions, spatial audio voice chat can maintain spatial awareness of collaborators. These two cues provide redundant information through complementary modalities.

\section{Activity visualization}

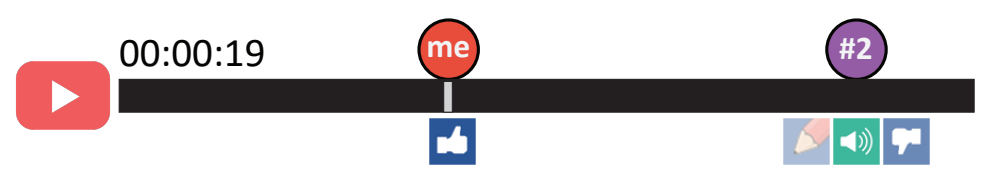

Figure 4.4: Activity icons such as thumbs up, thumbs down, speaking, and drawing are attached to the user's timeline position. These icons indicate user actions to all collaborators. They fade out over 3 seconds.

During in-person collaboration, people benefit from observing each other's reaction to the video. These reactions are perceived through implicit expressions (e.g., gasping, nodding, facial expressions) and explicit gestures (e.g., dragging the timeline, gesturing towards the video). The headset blocks awareness of others' reactions, so we provide a minimal interface for users to share their activities during review. More complex tracking systems could also be used to capture users' facial expressions and body language [68, 74].

Figure 4.4 shows our activity visualization. On the timeline, each user is represented as a color-coded icon, labeled with the user ID. Below each user icon are activity icons for 
speaking, thumbs up, thumbs down, and drawing. These icons appear when the activity occurs and fade out over three seconds. Each client sends its activity events to the server, so they can be shared with all other clients. The speaking event is detected by comparing the root mean square (RMS) energy [86] of the audio buffer with a threshold RMS which is calibrated by measuring a 5-seconds quiet period in the environment.

The buttons for drawing, thumbs up, and thumbs down are located above the timeline for easy access (Figure 4.1 p). A user can draw directly on the video to pinpoint specific details or explain spatial feedback to other collaborators. The thumbs up and down buttons complement drawing by providing a low-effort way to convey affect during playback. As described in our formative interviews, giving feedback on VR video can be difficult for nonexpert reviewers, especially when feedback occurs simultaneously with viewing. Moreover, observing thumbs icons on the timeline could also help collaborators monitor and coordinate effort in group work. Previous research found that similar features were well-received by users in a real-time collaborative search setting [80].

\subsubsection{View sharing}

As mentioned in our formative interviews, editors rely on sharing views in different ways during review. CollaVR provides specific tools to support these behaviors: the "peek" tool supports "looking over the shoulder," the "follow in time" tool enables "watching together," and the "slave" tool supports "coordinated viewing." These features are available on the "Peek" panel below the timeline (Figure 4.5).

\section{Peek tool}

Peeking enables users to quickly see each other's in-headset view. As shown in Figure 4.5, the Peek panel contains one thumbnail per collaborator, showing each persons' current view of the video. The server broadcasts every users' current timeline position and view direction, 


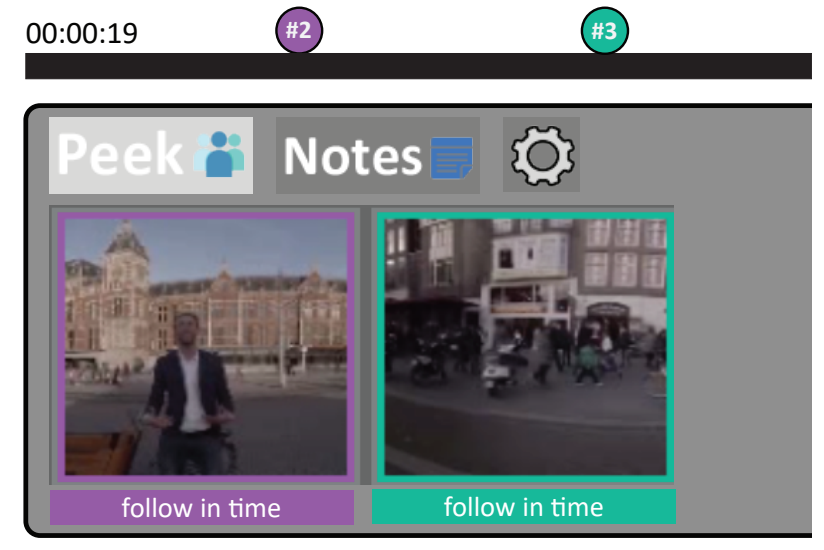

Figure 4.5: View sharing tools. When "Peek" is enabled, we display color-coded thumbnails showing each user's (e.g., \#2 and \#3) current video view. The user can further click "follow in time" or click on the thumbnail to trigger different view sharing modes.

and each client renders the thumbnail images accordingly. When a user hovers the mouse over a thumbnail, the corresponding user icon on the timeline is enlarged to emphasize that user's current temporal location.

\section{Follow-in-time tool}

Below each thumbnail is a "follow in time" button which allows a user to relinquish timeline control to a collaborator. Importantly, the user retains independent control of their gaze direction, so collaborators can coordinate and divide tasks [35]. For example, several editors can watch different directions of a video together to check for artifacts before publishing; searching for artifacts in a VR video can be tedious when done alone. When follow in time is active, a "Cancel" button appears above the timeline to restore normal viewing.

\section{Slave tool}

We also allow users to slave to a collaborator's view to emulate "coordinated viewing." Slaving synchronizes both the time and view direction of the user to a collaborator who is called the "master user" during this interaction. Thus, the slave user sees the exact same video image as the master user, allowing them to discuss fine-grained details or semantics of 
VR video such as peripheral vision, audience attention, or story [47]. To activate slaving, the user clicks on the thumbnail image and exits this mode with the "Cancel" button.

In our early experiments, we found that slaving could quickly cause simulator sickness [57] in the slave user. The slave user does not have control of their view, so when the head motion of the slave and master users differs, it creates conflicting motion cues for the slave user, leading to discomfort. This effect is related to using a gamepad to control the headset's orientation when playing VR games [116].

View vignetting can reduce discomfort [39, 84] but vignettes can occlude the peripheral details of the master user's view. We experimented with a variation of previous techniques [20, 116] in which we render semi-transparent moving particles over the scene, locked to the slave user's head motion. However, in the VR video review context, our pilot users found these particles too distracting and were often seen as video artifacts.

CollaVR employs a new visualization technique to reduce discomfort during slaving, while allowing the slave user to maintain a complete spatial awareness of the master user's view. The slave user's current view is dimmed, and a slightly scaled-down copy of the master user's entire field of view is rendered opaquely on top (Figure 4.6). This ensures the peripheral motion cues reinforce the slave user's self-motion perception [20], while the main visualization shows the slave user all of the master user's view.

\subsubsection{Feedback recording and browsing}

Because normal notetaking and feedback techniques are difficult in the headset, CollaVR supports capture and playback of feedback in the headset while also recording the context of the feedback. When the user presses the Record Note button on the timeline (Figure 4.1b), the user's gaze direction, speech, stroke drawing, and timeline control are all recorded until the user stops the recording. Automatic speech recognition is used to transcribe the user's speech when recording is finished, as this is much more convenient than typing in VR. 


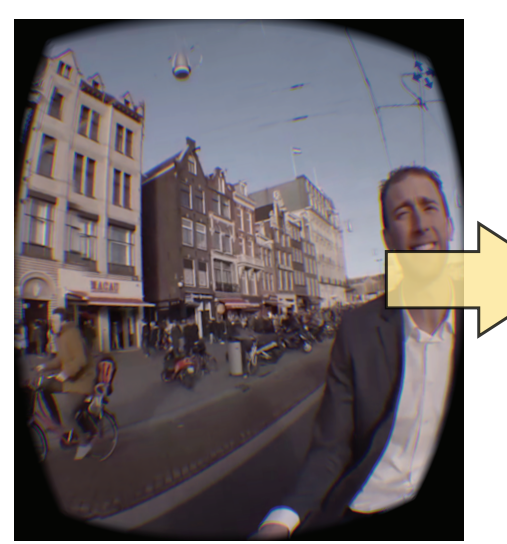

slave user's view

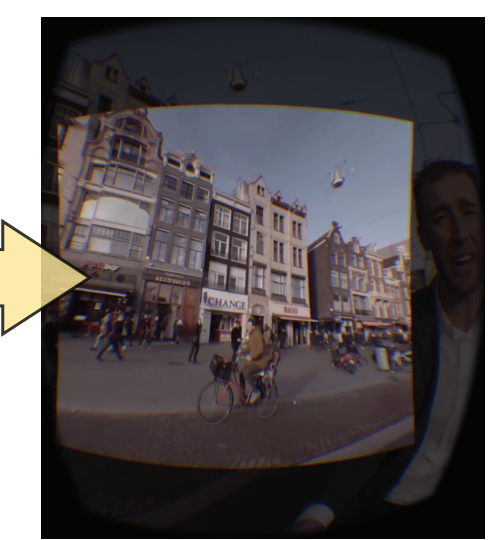

slave visualization

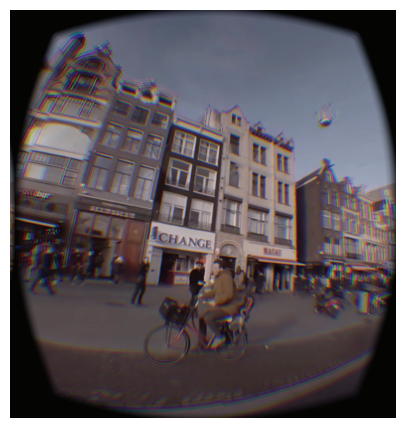

master user's view

Figure 4.6: Slave visualization. The master user looks at the bicycle rider (right). The slave user (left) uses "Slaving' to watch the master user's view. In this mode, the slave's own view is dimmed and a reduced-size copy of the master full field of view is rendered opaquely on top (middle). This allows the slave user to observe the master's actions while retaining peripheral self-motion perception.

Always-on recording could also be used, though this may entail privacy issues [118]. The recorded feedback is stored in a SQL database and the server signals clients to retrieve the newly added data, allowing collaborators to easily share feedback.

The "Note" panel (Figure 4.7) displays all recorded notes, color-coded by user. Hovering the cursor over a note shows the speech-to-text transcription of the user's comments, as well as the time interval that the note spans on the timeline. Clicking the note causes it to play back, and the viewer will see a recording of the original user's interactions. To reduce discomfort, the slaving visualization is also used here.

\subsection{User Study}

We conducted a preliminary expert review study to solicit feedback on how CollaVR supports collaborative VR video review. We chose a $2 \times 2$ within-subject design, comparing CollaVR with a baseline in a reviewing task.

Our study asked participants to critique VR video together and provide feedback for an editor. To reduce learning effects, we designed two tasks with two different videos. The 


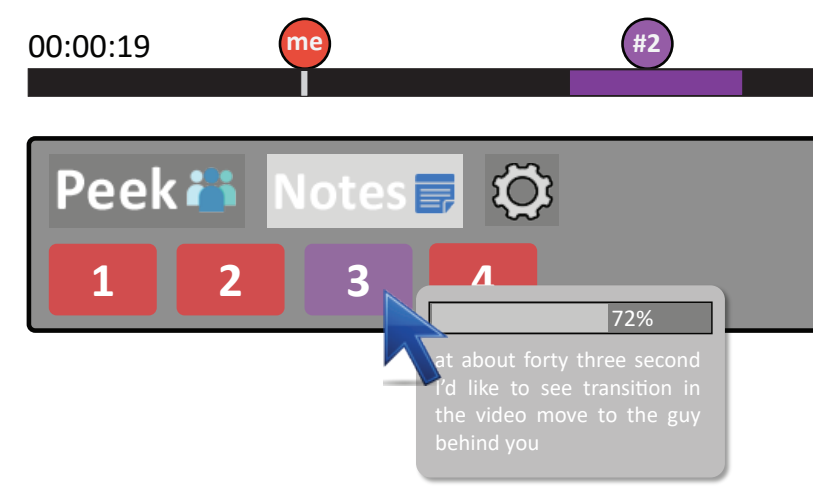

Figure 4.7: Recorded feedback is listed on the "Notes" panel, color-coded by author. A user can hover over a note to see its transcription and timeline interval. It also shows a progress bar when the user clicks on the feedback to review it.

videos were two documentaries, one depicting the story of a kite-surfer, and the other, a biking trip through Norway. Both videos were produced by the same aspiring videographer and were roughly equivalent in time (3:30 minutes) and editing style. Both videos also contained several technical issues such as shaky motion and stitching artifacts that motivate discussion.

The baseline condition is a stripped down version of CollaVR with all the collaboration tools removed (awareness visualization, view sharing, and notetaking). The remaining timeline interface allows viewing a video in a headset and also mirrored on a desktop monitor. Its design is similar to the current VR video players such as the GoPro player! Although CollaVR could be used with a desktop monitor, participants were encouraged to focus the discussion on the VR aspects of the video which require in-headset viewing. Since the baseline does not support notetaking, we gave participants pen and paper in that condition.

We invited five VR video professionals from three studios. These participants were not part of the formative interviews. They were divided into two groups of two to three people. Group 1 consists of a senior producer/panoramic imaging expert (P1), an editor/engineer (P2), and another editor/sound designer (P3) from the same studio. Group 2 consists of an

\footnotetext{
${ }^{1}$ http://www.kolor.com/gopro-vr-player/download/
} 
award-winning editor/cinematographer (P4) and a director (P5) from two different studios, but who have collaborated extensively before. Each group was assigned the task (kitesurfing or biking) and the system (CollaVR or baseline) following a counter balance order. Participants were given adequate training before each task. Participants took 5 minute breaks after each task to alleviate discomfort.

The study was conducted in a university research lab. Participants sat at computer desks in different corners of the room. The computers were connected via Gigabit Ethernet. One Oculus Rift CV1 and two DK2 headsets were used. The CV1 includes headphones and a microphone. The DK2s were paired with Logitech H390 headsets. Participants were each compensated with a $\$ 25$ gift card for their time.

\section{Measures}

Our main research question is how our system supports collaboration among users. We logged users' activities including voice chat, head tracking data, and tool usage. During the task, two researchers coded participants' conversation into a voice chat log. There is one entry per discussion, recording the timestamp, topic, and whether they used deictic or detailed references [46]. We only note spatial and temporal references. Deictic references use pronouns (e.g., "this," "that") or gaze-centered cues (e.g., "right where I'm looking"), while detailed references explicitly describe scene elements or timestamps.

After each task, we logged the total time and the number of recorded notes, and asked participants to fill out questionnaires individually. The questionnaire asked participants to rate self-perception of collaboration and how well the system supported them. Finally, participants listed their favorite features of the system and described their collaboration strategies.

We analyzed the log data to find times where two users shared the same video context, which is a basis for collaboration [103]. We adapted the measured shared focus metric in 
collaborative VR analytics research [29] to determine users' headset view similarity. Two views are considered similar if the angle between the head orientations is less than $40^{\circ}$ and the difference between the timeline positions is less than seven seconds. Then the headset view similarity is computed by dividing the total time two users have similar views by the total task time.

\subsubsection{Results}
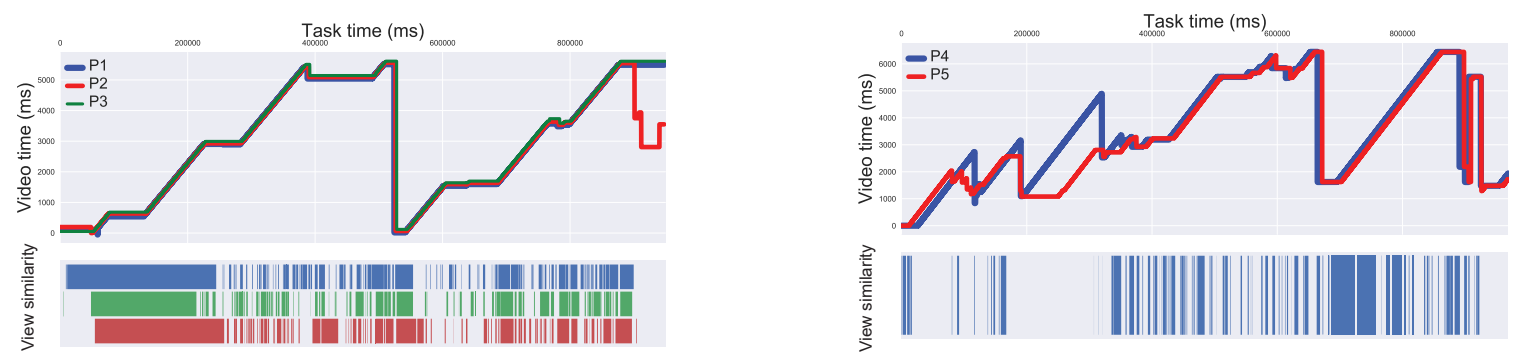

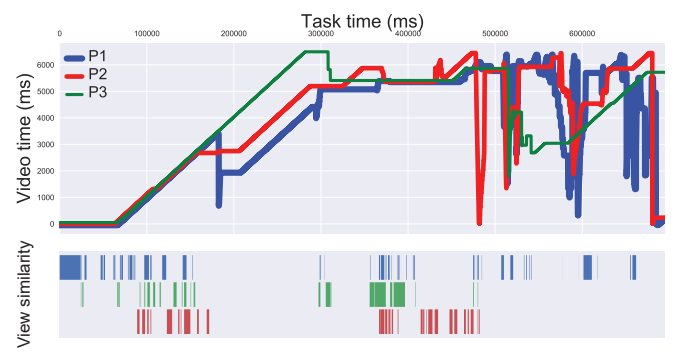

(a) Group 1: ours (top), baseline (bottom)

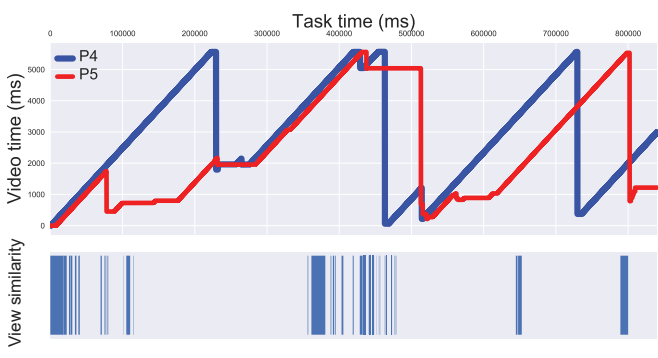

(b) Group 2: ours (top), baseline (bottom)

Figure 4.8: Video reviewing patterns (Video browsing time and Headset view similarity vs. Task time) of the two study groups. CollaVR enables useres to spend more time watching the video together (at the same time and in similar view directions). Note, in (a), view similarity is color-coded between pairs of users: blue (P1-P2), green (P2-P3), and red (P1-P3)

All groups were able to complete both tasks. Table 4.1 reports several performance statistics. Overall, groups using CollaVR spent more time on the task, engaged in more discussions, and aligned views more often. Participants were impressed by our interface, commenting that it would help alleviate collaboration problems they currently suffer in many stages of their work, including: having editors resolve issues with clients on the spot (P1, P2, P3, P4), discussing edits with other filmmakers (P3, P5), pitching a new story idea 
to a client (P3, P4), and dailies review (P5). We now examine participants' interactions in more detail.

\section{In-headset video reviewing}

Figure 4.8 visualizes the participants' reviewing behaviors. The Video time $\times$ Task time charts show that Group 1 (Figure 4.8 a) mostly watched the video together. They explained that their collaboration strategy involves discussing while watching together. CollaVR supports this via the "follow in time" feature, and the group assigned P3 as the controller with P1 and P2 following. They were unable to enact this strategy with the baseline. P1 started with a countdown so they could all press Play together. They watched the video together at first, but once somebody decided to pause or scrub the timeline, synchronization broke down. Toward the end of the task, each user browsed different parts of the video. The same pattern is apparent for Group 2 (Figure 4.8b). When using our system, P4 and P5 explored the video separately until they found something interesting. Then they synchronized playback, either manually using the timeline and gaze visualization as guidance, or through the view sharing tools. In the baseline, they made a small effort to synchronize playback, but mostly watched separately, occasionally sharing something interesting.

The View similarity $\times$ Task time charts show how long each group aligned their views (Figure 4.8). It is clear that CollaVR better supported view alignment than the baseline. The view similarity between P1-P2, P2-P3, P1-P3, and P4-P5 in our system are: 51.1\%, 27.6\%, $27.9 \%, 19.8 \%$, respectively, and in the baseline are: $14.0 \%, 9.8 \%, 10.5 \%, 7.1 \%$.

\section{Communication patterns}

Each participant conducted their review by finding and discussing details about the editing of the video. We grouped the discussion topics into 6 categories: stitching, stabilization, camera (water on the lens, rolling shutter), cinematography (cut continuity, pacing of a shot, 


\begin{tabular}{|c|c|c|c|c|}
\cline { 2 - 5 } \multicolumn{1}{c|}{} & \multicolumn{2}{c|}{$\begin{array}{c}\text { Group 1 } \\
\text { (P1, P2, P3) }\end{array}$} & \multicolumn{2}{c|}{$\begin{array}{c}\text { Group 2 } \\
\text { (P4, P5) }\end{array}$} \\
\hline Measures & Ours & Baseline & Ours & Baseline \\
\hline $\begin{array}{c}\text { Task time } \\
\text { (minutes) }\end{array}$ & 15.8 & 11.6 & 16.2 & 14.00 \\
\hline $\begin{array}{c}\text { Number of } \\
\text { discussions }\end{array}$ & 26 & 16 & 15 & 12 \\
\hline $\begin{array}{c}\text { Headset view } \\
\text { similarity (\%) }\end{array}$ & 35.5 & 11.4 & 19.8 & 7.1 \\
\hline Notes produced & 6 & 0 & 3 & 0 \\
\hline
\end{tabular}

Table 4.1: Results of the study. The headset view similarity of group 1 was computed by averaging the view similarity of each pair in the group.

clip orientation), visuals (logos, sharpness, color grading, lighting), and voice over.

When using CollaVR, groups discussed more than in the baseline (Table 4.1). We also counted the number of implicit and explicit spatial references used (Figure 4.9). Participants of both groups used more implicit references when referring video elements in CollaVR than the baseline, suggesting that our system enables participants to use the same natural verbal cues as in face-to-face interactions such as deixis and gaze [23].

In contrast to CollaVR, participants using the baseline required much more effort to establish common context. P5 mentioned in the post-study interview that "I can't just tell somebody to look at the upper left corner, because there is no corner, you can look anywhere." Moreover, using video elements for reference can lead to misunderstandings. In Group 1, P2 told P1 to look at the left side of a biker, but because the biker was facing the camera, her left was P1's right. P1 misunderstood and looked to the wrong side. P3 actively discussed the video when using CollaVR but said that when using the baseline, he "mostly watched quietly, allowing others to talk." In the baseline condition of Group 2, we counted 8 instances when a person initiated a discussion without any responses from their peers. These results are consistent with previous studies on social VR video watching [77, 109]. Without common conversational context, users' communication suffers. 


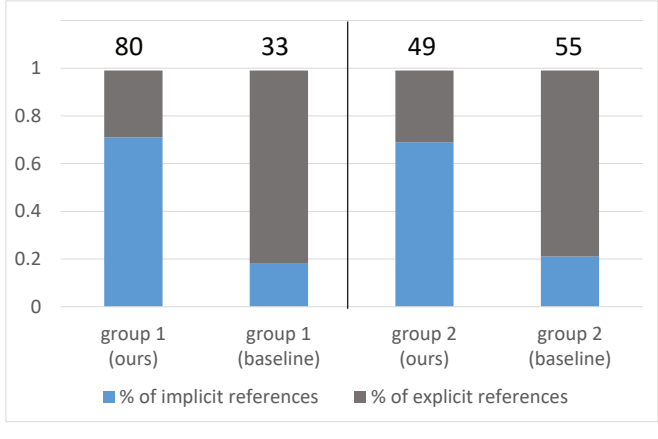

Figure 4.9: Communication patterns, shown as fraction of implicit and explicit references uttered by study participants. The numbers above the chart show the total number of references made in that condition.

\section{Subjective feedback}

Figure 4.10 a shows participants' responses about perceived comfort (Q2) and collaboration of each system (Q1, Q3, Q4, and Q5). None of the participants reported any symptoms of motion sickness after the study. However, Q2 ratings for our system were slightly lower than the baseline. Q2 does not distinguish between physical comfort and ease-of-use however. P1 and P4 rated Q2 neutral (4) and said they felt a bit overwhelmed to learn all of CollaVR's tools, but they loved the familiarity of the 2D GUI design and thought it would not take long to master. On all four collaboration questions, participants rated CollaVR higher than the baseline.

We also asked users to rate the each features' helpfulness in supporting collaboration (Figure 4.10p). Most features received high ratings. Users named their favorite features as: drawing (P1, P2, P3, P5), viewport visualization (P1, P3, P5), follow in time (P1, P3, P5), slaving (P1, P4, P5), peek (P4, P5), and record/view notes (P1, P2, P4, P5).

Although all participants appreciated voice chat, opinions differed about spatialized audio. Group 1 rated it lower, and P1 and P2 said they did not pay much attention to it. Figure 4.8 a shows they mostly aligned views, so the spatial cues may have not been useful. In contrast, Group 2 favored this feature. P4 explained it helped him find collaborators without having to think about it. P5 found an unexpected creative use: he sometimes 


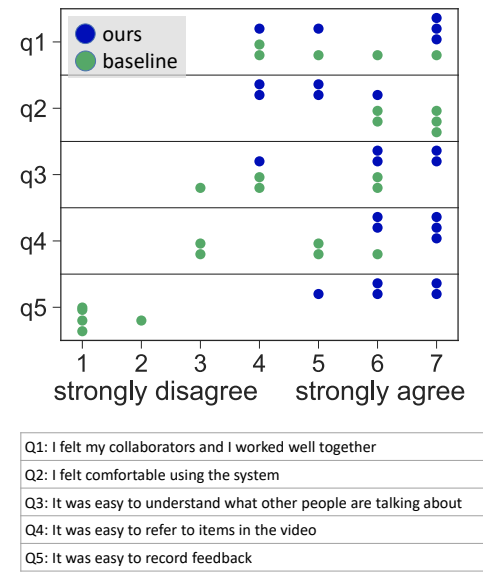

(a) Post-task questionnaire

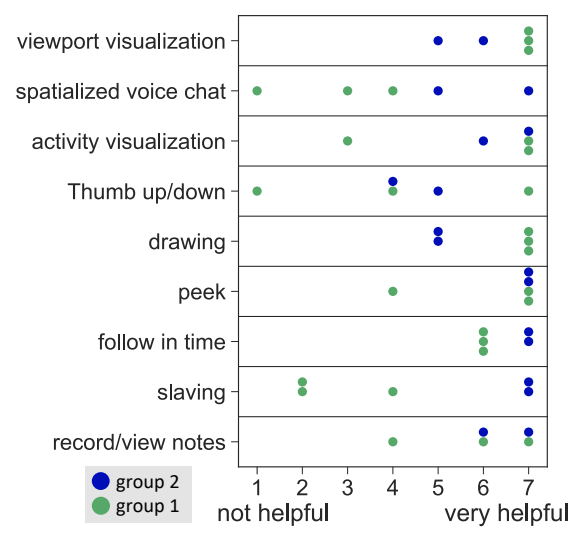

(b) Subjective feedback on our system

Figure 4.10: Results of the post-task questionnaire (a) and subjective feedback (b) on individual features of our system. Each $\operatorname{dot}(\bullet)$ is a rating of participant on a 7 -point Likert scale.

left the Peek panel open, so he could monitor the video through the collaborator's view and infer its direction from the spatialized audio. Such monitoring is common in group collaboration [112].

Slaving also received diverging ratings. Group 1 gave low scores. P1 and P2 slaved to P3 initially, but since both were active in the discussion, they quickly switched to follow-in-time so they could look around freely. Group 2 rated slaving highly. As a cinematographer, P4 mentioned he could use it to point out both scene details and abstract content like flow or story. P5 praised the visualization, saying that he was surprised it did not make him sick. As a director, P5 deemed this feature as very beneficial as he could have editors locked to his view during review.

Finally, participants found feedback recording very helpful, though they used it differently. In Group 1, P3 recorded the entire session for the whole team, while P2 recorded important details. In Group 2, each participant recorded feedback independently. Participants emphasized that this feature could also be useful for offline (asynchronous or remote) collaboration. Participants using the baseline stayed in-headset the majority of the time and did not take notes on pen and paper. 


\subsection{Discussion and Limitations}

Our study shows that CollaVR's features enable expert users to collaboratively review VR video relatively unhindered. Compared to the baseline system without any collaboration support, participants using our system spent more time with aligned views and were able to discuss with more deictic references. Watching video together, gesturing, and talking are all natural in-person interactions. Our results suggest the awareness visualization and view sharing tools of CollaVR help users establish common context to discuss videos inheadset, akin to face-to-face collaboration. This is important because understanding VR video requires people to experience it in-headset, and our system helps filmmakers share that experience and exchange ideas within the medium.

The additional collaboration support of CollaVR might have aided participants' reviewing performance. Compared to baseline, they spent more time in the task and initiated more group discussion about VR video editing techniques, focusing on in-headset experiences such as visuals and story flow that would normally be difficult to describe on the desktop. Participants also engaged in collaborative notetaking. They used our feedback recording tool to coordinate the notetaking task, or share notes with each other, all in the headset. These results are encouraging and motivate more explorations of collaboration techniques to support in-headset reviewing.

Our study also confirms that multimodal recording is promising to capture interactions in VR video, consistent with previous research on collaborative review [87, 118]. Participants highlighted its potential not only in notetaking, but also in capturing the entire collaboration session and in asynchronous review. Exploring filmmakers' needs for recording and visualizing feedback is interesting future work [6, 70].

CollaVR could be extended to other VR applications. For example, it could enable large groups to watch VR video together, simulating a virtual living room or cinema. To support more users, our awareness visualization could adapt to the individuals rather than 
the group [44]. Or, our features could be integrated with other in-headset production tools such as Vremiere [84] to simultaneously support reviewing and editing, similar to how editors discuss and try alternatives when reviewing regular video [87].

We conducted our exploratory study in a lab. To better understand CollaVR's real performance requires deploying it in actual studios. Rather than using local video files, we could stream video from an editing suite, enabling editors to discuss drafts more easily. Better audio compression techniques are needed to improve voice chat performance. Stereoscopic VR video is common and requires special treatment of UI elements [48]. To be more widely useful, we could support low-end headsets such as Google Cardboard and Samsung GearVR as well.

\subsection{Conclusion}

We present CollaVR, an in-headset interface for collaborative VR video review. Our core contribution is a set of techniques that support multiple users to watch VR video, exchange feedback, and take notes together without being hindered by VR headsets, by reproducing the benefits of natural face-to-face interactions. Our preliminary expert review study showed that filmmakers are positive about the potential to review VR videos in CollaVR over a baseline interface. These results highlight the potential of VR video as a collaboration space that we have taken only one step in exploring. 


\section{Depth Conflict Reduction for Interfaces in Stereoscopic VR Video}

As demonstrated in Vremiere and CollaVR, VR video authoring can benefit tremendously from a WYSIWYG workflow in VR. However, these explorations have focused only on monoscopic video. Integrating application interfaces into stereoscopic VR video can cause undesirable perceptual problems. Most video applications render user interface (UI) elements on top of the video. But in stereoscopic video, the perceived depth varies over the image. Thus, the perceived depth of the video can conflict with that of the UI elements, creating discomfort and making it hard to shift focus.

In this chapter, we address this problem by exploring two new techniques that adjust the UI rendering based on the video content. The first technique dynamically adjusts the perceived depth of the UI to avoid depth conflict, and the second blurs the video in a halo around the UI. We conduct a user study to assess the effectiveness of these techniques in two stereoscopic VR video tasks: video watching with subtitles, and video search.

\subsection{Motivation}

Compared to monoscopic VR video, stereoscopic video provides a much greater sense of immersion. It is becoming increasingly available due to many recent advances in camera technology [2, 76], and new processing tools [58] and editing interfaces [84, 83] are being

developed for these videos. These videos have led to interesting applications beyond film production such as data visualization [25], virtual tours[36], and free-viewpoint video [51].

However, the extra sense of depth in stereoscopic VR video can be problematic to users of VR video applications. Common user interface (UI) widgets like video navigation, 

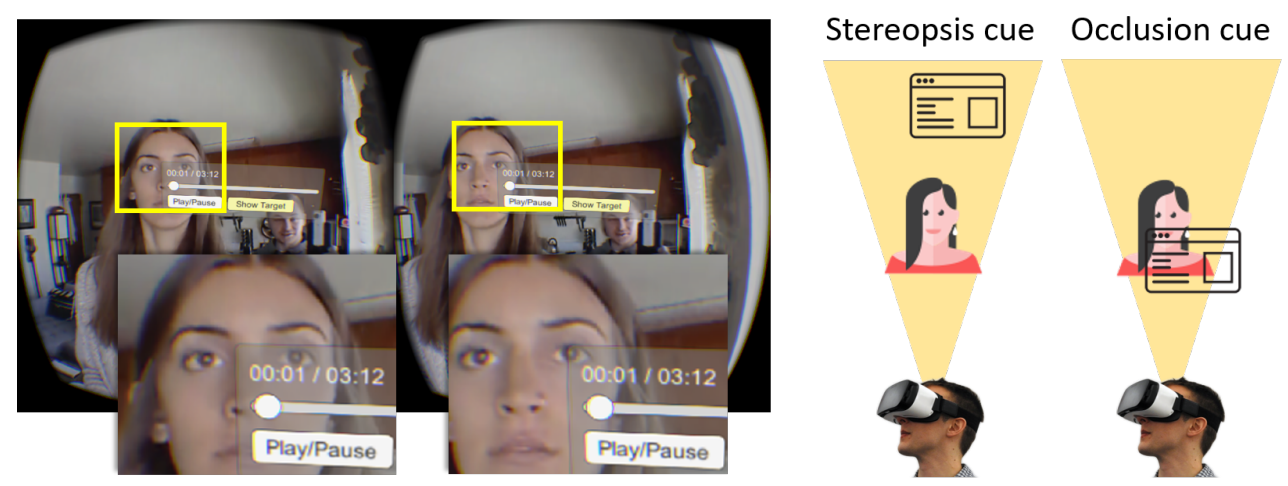

Figure 5.1: Depth conflict illustration. An interface (the video player) overlays a video object (the actress) but is actually behind her in depth. Left: The resulting graphics are uncomfortable to view in VR (e.g., the areas behind the text 00:01 in the insets are different between the left and right views). Viewers may also experience difficulty changing focus between the interface and the video. Right: illustration of the conflicting depth cues perceived by the same viewer in the same view. (O) Kevin Kunze

subtitles, annotations, and tool palettes are often rendered on top of the video. Each widget must be rendered at a specific perceived depth, which is controlled by varying the disparity (difference in horizontal position) between the left and right eye views. However, the perceived depth of the stereoscopic VR video varies greatly, which can create a conflict in perceived depth. Specifically, when a UI element is rendered over a video element that is perceived to be closer than the UI element (Figure 5.1), there is a conflict between the stereopsis depth cue and the occlusion depth cue. In other words, objects in the video are blocked by a widget which is behind the video objects. This creates visual discomfort [114, 63] and confusing visual cues. Also, alternating eye focus between the video and the UI can be difficult when the difference in depths is large. A naive approach to these problems is to place the UI very close to the viewer. However, prolonged exposure to close objects in VR is uncomfortable [59, 65] and still does not solve the problem completely: one cannot move the UI close enough to never conflict with video objects.

A key insight of our work is that, because conflicts occur at the intersection of the UI elements and the video, they can be resolved by local adjustments: either by adjusting the UI, or adjusting the video locally around the UI. 
Based on this insight, we introduce two techniques for reducing these depth conflicts. The first, Dynamic Depth, dynamically adjusts the depth of UI widgets so that they normally rest at a comfortable default depth, but move closer to the viewer (by changing disparity) when video elements would conflict. The apparent depth of video elements is precomputed by a stereo computer vision algorithm. We also introduce a simpler technique, Halo Blur. Halo Blur simply blurs the video around the UI. While this does not necessarily produce a geometrically-valid configuration, it is very simple to implement and can mask highfrequency depth cues from stereo images [34, 49], thus potentially reducing depth conflicts and helping ease focus on the interface. Our techniques are local, fast, and do not estimate eye gaze, and therefore could be used on low-end HMDs.

We evaluate our techniques in a preliminary within-subject user study with two video tasks: watching videos with subtitles and video searching. We examine performance data and subjective ratings of the participants to assess how depth conflicts affected their experience. We find that Dynamic Depth is preferred over the baseline condition and over Halo Blur.

\subsection{Depth Conflicts in VR Video Interfaces}

In this section, we identify three sources of perceptual conflict that can happen when UIs overlap stereoscopic VR video. Current VR HMDs typically use stereoscopic display to convey depth perception [59]. These displays render slightly different views to the left and right eyes, allowing the viewer to see depth through the stereopsis process [50]. The human visual system also perceives depth through other cues such as occlusion, motion, texture, or perspective. Viewers may experience discomfort and perceptual difficulties when different depth cues are perceived simultaneously. We use the term "conflict" loosely to mean incongruent depth cues.

Occlusion/stereopsis conflict. UI widgets are normally rendered on top of video, indicating that the UI is in front of the video. The UI and stereoscopic video are each 
rendered with disparities that creates perceived depth. If the UI disparity indicates that it is further away than the video elements, then the cues conflict because it is physically impossible for an object to be occluded by another object that is behind it (Figure 5.1). Since occlusion is one of the strongest depth cues [65], viewing video objects that are closer in depth but behind the UI can cause discomfort and some forms of double vision, e.g., the viewer sees two images of either the UI or the video [79, 63].

Near/far conflict. Some applications, like video editing, require frequent shifts of attention between the UI and the video. When the UI and the video are perceived at very different depths, the viewer's eyes have to re-verge when transitioning from one element to the other. Alternating eye vergence can be cumbersome and may cause eyestrain [104, 64].

Pictorial conflict. Some textures of the video around or behind the interface can be seen very differently between the left and right eyes (Figure 5.1 Left). The resulting graphics can create binocular rivalry [117]. Although binocular rivalry is a natural phenomenon, it is more prevalent in consumer stereo displays because they cannot render natural depth-of-field blur [49]. This phenomenon can make it difficult for the viewer to fuse the stereo images of the video, or properly focus on the interface. Moreover, many VR applications typically render the UI semi-transparent to prevent blocking too much of the content underneath. Semi-transparent UI can potentially worsen the effect of binocular rivalry [66].

\subsection{Techniques Considered}

We built a generic $360^{\circ}$ stereoscopic video application environment in VR to experiment with our techniques. To enable stereoscopic playback, the left and right images of each frame are texture mapped on two 3D spheres, each of which is rendered to the left and right views of the VR HMD, respectively. The camera baseline is set to the viewer's interpupillary distance (IPD). UI elements are rendered on top of the video. We consider the UI as a 2D WIMP-style panel (Windows Icons Menus Pointers) because it is most relevant to video 
applications. A panel can contain various widgets such as texts, buttons or sliders. The UI can be rendered either in display-fixed (e.g., advertisement banners, compass, subtitles) or world-fixed mode (e.g., tool palettes, annotations, gaze triggers) [38]. Our video application is developed in Unity3D for the Oculus Rift CV1 HMD. Users use a 6DOF controller to interact with the interface.

\subsubsection{Baseline}

The baseline technique we consider is to place the UI at a fixed depth of three meters from the viewer, as suggested by the Oculus design guidelines [1]. Placing the UI even closer than that could further reduce depth conflicts, but would also increase oculomotor discomfort [65].

\subsubsection{Dynamic Depth}
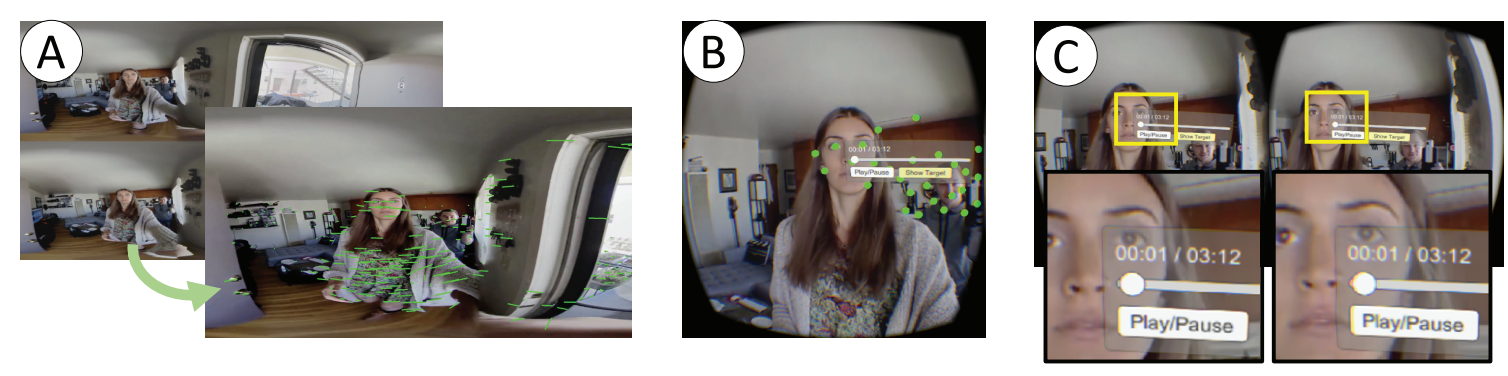

Figure 5.2: Overview of Dynamic Depth. (A) We pre-process the input video to find feature points and left/right disparities (e.g., green lines on the actress) (B) Features points are mapped to the VR view and shown as the green dots (only for illustrative purposes). Dynamic Depth estimates the perceived depth of the video based on these points. It detects when depth conflict occurs by comparing the depths between the UI and the video. (C) Dynamic Depth moves the UI closer to the viewer to reduce depth conflicts. Notice in the insets that the areas around the interface's corner are more geometrically consistent compared to the same scene in Figure 5.1. (C) Kevin Kunze

At a high level, Dynamic Depth detects and resolves depth conflicts by adjusting the perceived depth of the UI so that it appears at the same depth of the nearby video content (Figure 5.2). This requires first estimating the perceived depth of the video. We also limited 
the rate at which the rendered depth of the UI changes, in order to avoid distractingly-fast changes.

\section{Disparity map pre-computation}

We first pre-process the input video to estimate dense correspondences between the left and right view in each frame using an optical flow method [88]. The resulting flow vectors give the disparities of each pixel in the video frame. A set of left/right feature points and their corresponding disparities are selected for the real-time conflict detection step (Figure 5.2A).

\section{Potential conflict detection}

Based on the disparity maps, Dynamic Depth detects depth conflict by comparing the perceived depth of the video and the UI elements in VR.

We approximate the perceived depth of the video by analyzing the region of the disparity map that the UI currently overlays. Feature points from the disaprity map are converted to spherical coordinates in the video spheres, so we can compare the position of the UI in VR to any of these points. Then, we select a subset of points within $30^{\circ}$ from the UI's center (Figure 5.2B). Each point maps to a video feature around the UI and also contains disparity information. Following the approach of Blum et al. [12], the screen disparity $d$ of a feature point is:

$$
d_{i}=\left(p_{\text {focus }, L}-p_{\text {focus }, R}\right)-\left(p_{i, L}-p_{i, R}\right)
$$

where $p_{\text {focus }}$ is the focus point of the VR HMD (e.g., the 3D point where the disparity is zero) and $p_{i}$ is the feature point that the viewer is looking at. $L$ and $R$ denotes the screen coordinates of these points in the left and right cameras. Smaller negative $d$ indicates video objects that seem closer to the viewer, while positive $d$ indicates objects that are in focus or seem farther away.

Thus, at any moment in time, we can compute the perceived depth of the video region 
around the UI (denoted as $d_{\text {Video }}$ ) by aggregating $d$ values of the nearby feature points. To account for variations in depth, we extract the top $10 \%$ smallest $d$ values and find the median $d$ value. The data is then filtered with a moving window from the last 10 data points to reduce noise. We focus on small $d$ values because closer video objects can attract more attention [113]. They also take up more space in the view and are more likely to cause conflicts. The final smoothed disparity value $\left(d_{V i d e o}\right)$ gives a proxy to estimate the perceived depth of the video around the UI.

To determine when depth conflicts occur, we also compute the screen disparity of the UI's center point $\left(d_{U I}\right)$ using Equation 5.1. We consider a conflict to occur when the absolute difference between $d_{V i d e o}$ and $d_{U I}$ is larger than a threshold $t=5$ pixels. This value is chosen empirically so that Dynamic Depth can quickly trigger the next depth adjustment step. We used an absolute difference to handle both cases when the perceived depth of the UI is behind or in front of the video.

\section{UI depth adjustment}

If there is no depth conflict, the UI is placed at the default depth. Otherwise, Dynamic Depth adjusts the UI's depth to minimize the difference between $d_{V i d e o}$ and $d_{U I}$, which in turn can resolve the conflicts. However, changing the UI's 3D coordinates would require additionally adjusting scaling, collision detection, and rendering order parameters. Thus, we followed the approach of Oskam et al. and change $d_{U I}$ by shifting the left and right camera frustums horizontally [85]. Briefly, shifting the frustums inward makes the UI appear closer and vice versa (Figure 5.2C). These cameras are used to render the UI independently from the video, so the disparity shift does not affect the depth perception of the video.

An important consideration of camera shifting is the rate of change of the UI's depth (denoted as $\delta$, measured in arcmin/s). Shifting the frustums too quickly can be distracting and uncomfortable [108], while shifting too slowly will make the UI unable to resolve 
depth conflicts in a timely manner. To balance this tradeoff, we adjust $\delta$ using a number of heuristics determined from a pilot study with four test users.

Default rate. First, we set the default $\delta$ values to be $60 \mathrm{arcmin} / \mathrm{s}$ when the UI needs to move closer to the viewer and $30 \mathrm{arcmin} / \mathrm{s}$ when it recedes. They were chosen so that the UI would move closer in depth faster when it conflicts with video elements. Otherwise, the UI does not need to act fast when there is no depth conflict or when video elements are far away, and so it can recede more slowly.

Speed up. Second, we speed up these rates by a factor of 10 when the UI's position changes quickly. Users often move the UI, either through head motion or through the hand controller. When the position changes quickly, such as when the user looks around for inspection, it is highly unlikely that the user would notice any changes in the UI because of change blindness phenomenon [11]. We leverage these opportunities to quickly adjust the UI depth without sacrificing comfort. We set the speed threshold to be $7 \mathrm{~m} / \mathrm{s}$ based on the pilot tests.

Limits. Finally, we limit the UI depth near the comfort zone suggested by the HMD manufacture guideline [1] to avoid causing excessive uncomfortable disparities.

\subsubsection{Halo Blur}

We also explore an alternative, simpler technique that does not require the estimation of the video disparity map. Our Halo Blur technique applies blur effects to the video images around the UI (Figure 5.3). The blur effect masks high-frequency spatial data from images and thus could potentially weaken the stereopsis cue or reduce binocular rivalry [34, 49]. Thus, Halo Blur could help reduce depth conflicts and ease the eye transition between the UI and the video. Compared to Dynamic Depth, it also does not require estimation of depth maps or shifting the UI in distracting ways.

To achieve the blur effect, we add an additional blurred texture canvas below the UI 


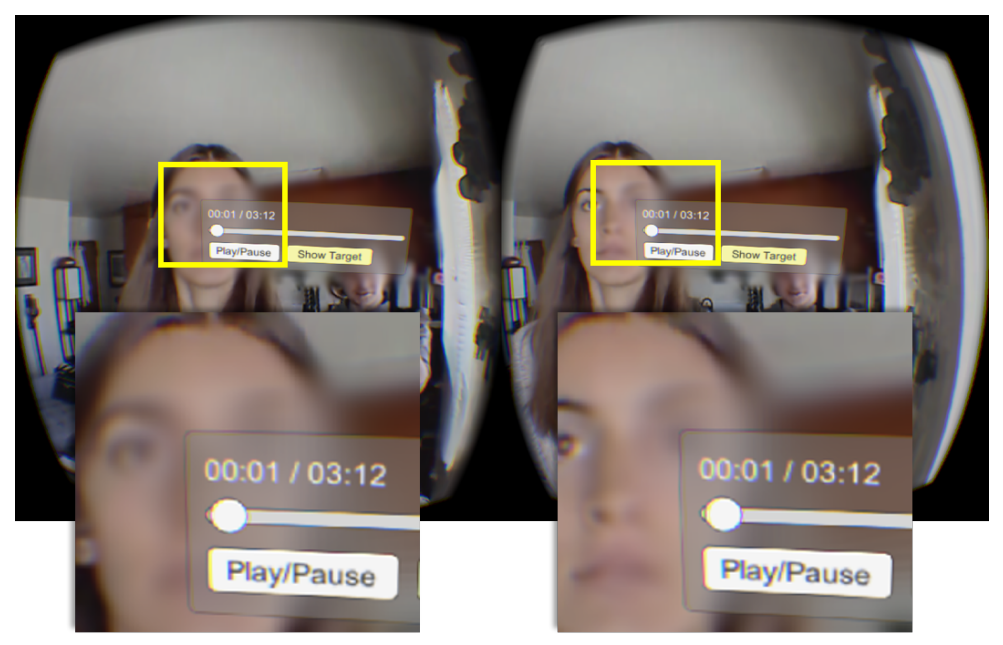

Figure 5.3: Halo Blur blurs the video content around the UI. Insets: compared to the same scene in Figure 5.1, the current scene is still not geometrically consistent. However, the blur effects mask high-frequency spatial information in the video images and makes the details from the UI clearer. (C) Kevin Kunze

pane. The canvas is slightly bigger than the UI. For each pixel in this canvas, a fragment shader looks up the color values of the video image textures from the previous rendering passes. The shader then applies a Gaussian blur kernel to each pixel, varying the strength of the effect so that it is strongest in the center and gradually diminishes outward.

\subsection{User Study}

We conducted a preliminary user study to evaluate our two techniques in two test VR video applications. Participants were asked to perform two common video tasks: watching video with subtitles (Subtitles) and searching for a target video scene in VR (Search). These tasks allowed us to examine our techniques in different application scenarios.

\subsubsection{Tasks}

In the Subtitles task, participants were asked to watch videos with subtitles. We lowered the video volume to 5\%, so this task would demand high attention focus to follow the video's narration or dialogue. Participants were also explicitly instructed to watch the video and read 
the subtitles. The UI was rendered as a text box that follows the user's head movement (e.g., display-fixed) and was semi-transparent. Participants could adjust the vertical placement of the text box using a thumbstick controller.

In the Search task, participants were asked to search for a target scene as quickly and accurately as possible. The target scene is shown as a thumbnail on the UI. In comparison to the Subtitles task, this task requires rapid attention shifts between the UI and the video. The UI is a basic video player timeline interface, including a seek slider, a play/pause button, and another button that shows or hides the target thumbnail. The UI was rendered in world-fixed mode and was fully opaque. Participants could use a controller to move the UI or interact with the buttons.

To reduce learning effects in the study, we used six different videos. The details of these videos are summarized in Table 5.1. Five of them captured scenes using static cameras. One video (Hemophillia) contains a 3-second segment with extreme camera motions. We dimmed this segment to prevent motion sickness. These videos were selected because they contain many scenes with multiple objects that appear close to the viewer and thus can induce depth conflicts.

\begin{tabular}{|c|c|l|}
\hline Video & Task & \multicolumn{1}{c|}{ Description } \\
\hline Party1 [33] & Subtitles & $\begin{array}{l}\text { Actors perform a scene around the camera and speak } \\
\text { to the viewer. (2:05 min) }\end{array}$ \\
\hline Party2 [33] & Subtitles & The second part of Party1. (2:05 min) \\
\hline Hemophilia [28] & Subtitles & $\begin{array}{l}\text { A narrator explains Hemophilia as the camera moves in a 3D } \\
\text { scene of human blood veins (2:21 min) }\end{array}$ \\
\hline Gladiator [43] & Search & $\begin{array}{l}\text { Two gladiators fight around the camera. The target scene } \\
\text { is set at the beginning of the fight. (2:05 min) }\end{array}$ \\
\hline Proposal [30] & Search & $\begin{array}{l}\text { Two main actors and a few film crews surround the camera. } \\
\text { The target scene is set at a unique pose of the two actors. } \\
\text { (1:26 min) }\end{array}$ \\
\hline Circus [32] & Search & $\begin{array}{l}\text { Six artists perform around the camera. The target scene is } \\
\text { set at a unique pose of the three artists. (2:34 min) }\end{array}$ \\
\hline
\end{tabular}

Table 5.1: Brief descriptions of the video materials used in the study. 


\subsubsection{Experiment design \& procedure}

We recruited 12 participants for the study (10 males and 2 females ranging in age from 19 to 26 with a mean age 23.3) from a university. Four participants had not experienced VR before. The rest reported limited experience. We checked to make sure participants could see stereoscopic 3D with a few test scenes in the HMD. Three participants wore glasses during the study. We calibrated participant's IPD using the HMD's built-in tool (mean = $64.58 \mathrm{~mm})$.

We chose a within-subject study design. Our independent variable was Technique (Dynamic Depth, Halo Blur, and Baseline). With two tasks and three techniques, each participant performed 6 trials. After each trial, participants filled out a questionnaire form with questions about symptoms of depth conflicts and their subjective preference (Table 5.2). We also recorded task time and task error in the Search task. The timer starts when a participant selects the "show target" button, and ends when the show target button was selected again. The Task error is the absolute difference between the target time and the participant's time (in seconds). We also measured Simulation Sickness Questionnaire (SSQ) scores [55] throughout the study to observe participants' comfort level.

\begin{tabular}{|c|l|}
\hline \multicolumn{2}{|c|}{ Subjective Questionnaire } \\
\hline $\begin{array}{c}\text { Q1 } \\
\text { (Focus switch) }\end{array}$ & $\begin{array}{l}\text { How easy was it to change focus } \\
\text { between the UI and the video? }\end{array}$ \\
\hline $\begin{array}{c}\text { Q2 } \\
\text { (Legibility) }\end{array}$ & $\begin{array}{l}\text { How easy was it to view the } \\
\text { information on the UI? }\end{array}$ \\
\hline $\begin{array}{c}\text { Q3 } \\
\text { (Double vision) }\end{array}$ & $\begin{array}{l}\text { To what extent did you notice } \\
\text { double images of the same object? }\end{array}$ \\
\hline $\begin{array}{c}\text { Q4 } \\
\text { (Distraction) }\end{array}$ & $\begin{array}{l}\text { To what extent did you think the } \\
\text { UI was distracting during the task? }\end{array}$ \\
\hline $\begin{array}{c}\text { Q5 } \\
\text { (Preference) }\end{array}$ & $\begin{array}{l}\text { To what extent did you prefer } \\
\text { this condition? }\end{array}$ \\
\hline
\end{tabular}

Table 5.2: Subjective questionnaire. Participants reponded with a 7-point Likert scale, ranging from very difficult to very easy $(\mathrm{Q} 1, \mathrm{Q} 2)$ and not at all to very much $(\mathrm{Q} 3, \mathrm{Q} 4, \mathrm{Q} 5)$.

The study was conducted in a university lab. Participants were first explained the 
procedure. Then, they performed the Subtitles and the Search task in order. Before each task, participants practiced the task using a test video for 5 minutes and filled a pre-SSQ form. In each task, the order of techniques was counterbalanced using a Latin square design. After each trial, participants filled out post-questionnaires and SSQ forms. To reduce carry-over effect, they were allowed to rest for 5 to 10 minutes between tasks. The study lasts about 45 minutes.

\subsubsection{Results}

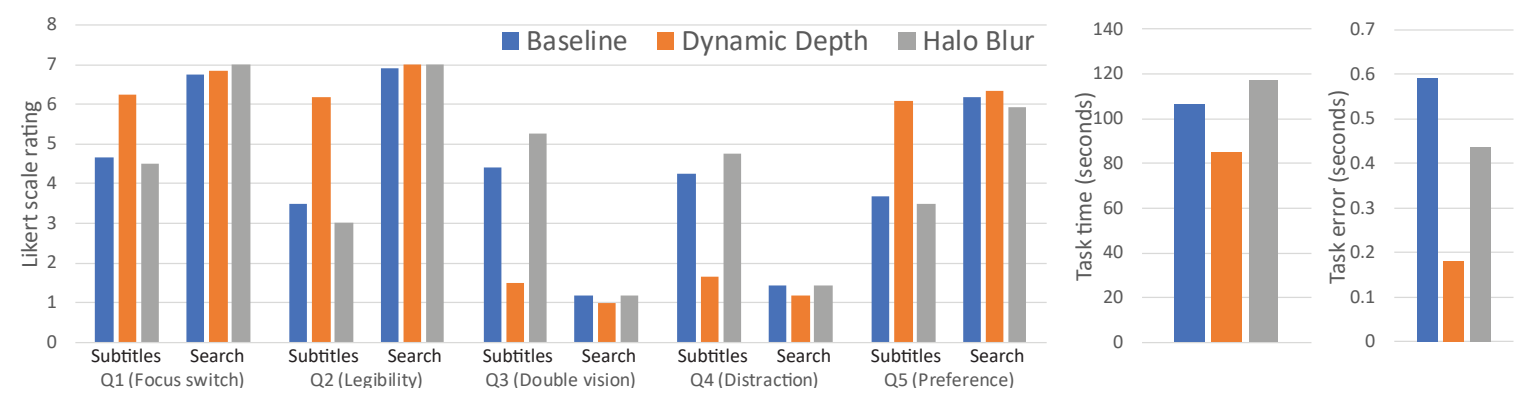

Figure 5.4: (Left) Summary of participants' ratings to the subjective questionnaire in both tasks. (Middle and Right) Task time and task error summary of the Search task.

All participants completed the study without any noticeable signs of motion sickness. In each task, each participant filled the SSQ questionnaire before the task and after each trial, resulting in 8 data points. They are: before task $1(M=0.91, S D=1.08)$, after trial $1(M=1.08, S D=0.99)$, after trial $2(M=1.08, S D=1.31)$, after trial $3(M=3.0, S D=$ 4.49), before task $2(M=0.5, S D=0.79)$, after trial $4(M=0.33, S D=0.65)$, after trial $5(M=0.33, S D=0.65)$, after trial $6(M=0.66, S D=0.98)$. The ratings after each trial rise slightly compared to the rating before the task. We analyzed the differences using a paired-samples $t$-test and found that none of them was statistically significant $(p>0.05)$.

We then analyzed the subjective questionnaires to examine if participants experienced symptoms of depth conflicts. We used Friendman's test. Post-hoc analysis was done using Wilconxon signed rank tests. We applied Bonferroni correction to adjust for multiple testings 
and used an alpha level of 0.05. Figure 5.4 summarizes the ratings in both tasks.

In the Subtitles task, participants experienced various problems from depth conflicts. Participants gave Dynamic Depth better ratings in all questions. There were significant differences for all except Q1. We report the results below:

Q1 (Focus switch) $\left(\chi^{2}(2)=5.31, p>0.05\right)$. Participants who used Baseline and Halo Blur reported having difficulties changing eye focus, especially when the pace of the subtitles is faster in the Hemophilia video. We observed that 3 participants used an ad-hoc solution to reconcile the problem: they quickly find and turn to a farther scene elsewhere in order to make the switch easier. Thus, they did not rate the problem negatively.

Q2 (Legibility) $\left(\chi^{2}(2)=15.59, p<0.01\right)$. Participants who used Baseline and Halo Blur reported that they were not able to read the text when the actors or the blood cells in the video are nearby. Two participants mentioned that if they squinted their eyes then they could read it, but it was very cumbersome. In Dynamic Depth, none of the participants reported any problems. Only one participant reported that the subtitle "seems a bit too close", but it was still easy to read. The differences between Dynamic Depth and Baseline $(Z=-3, p<0.05)$ and Halo Blur $(Z=2.94, p<0.05)$ were statistically significant.

Q3 (Double vision) $\left(\chi^{2}(2)=15.95, p<0.01\right)$. When asked to describe why the subtitles were difficult to read, most participants who used Baseline and Halo Blur reported they saw two overlapping images of the subtitles. The double images made it very difficult to focus on the text. Double images did not occur in the Dynamic Depth condition. The differences between Dynamic Depth and Baseline $(Z=-2.6, p<0.05)$ and Halo Blur $(Z=3.08, p<0.05)$ were statistically significant.

Q4 (Distraction) $\left(\chi^{2}(2)=17.7, p<0.01\right)$. Participants who used Baseline and Halo Blur found that it was quite distracting when they could not read the subtitles. In Dynamic Depth, the ratings for this question are not as high. The differences between Dynamic Depth and Baseline $(Z=-2.85, p<0.05)$ and Halo Blur $(Z=3.07, p<0.05)$ were statistically significant. 
Q5 (Preference) $\left(\chi^{2}(2)=16.5, p<0.01\right)$. Finally, all participants gave higher preference ratings to Dynamic Depth. The differences between Dynamic Depth and Baseline $(Z=2.95, p<0.05)$ and Halo Blur $(Z=-2.95, p<0.05)$ were statistically significant.

In the Search task, the differences in all questions were not statistically significant. None of the participants reported any visual problems or artifacts related to depth conflicts. We further looked into the performance data of the Search task (Figure 5.4). The task time and task error data were analyzed using repeated-measures ANOVA. Overall, participants in all conditions were accurate in finding the target scenes. The differences in task error were not statistically significant $(F(2,22)=0.49, p>0.05)$. Participants who used Dynamic Depth were slightly faster than the other two conditions, but the differences were also not statistically significant $(F(2,22)=3.14, p>0.05)$.

\subsection{Discussion and Limitations}

In Baseline, participants' ratings about depth conflicts were different between the two tasks. In the Subtitles task, participants reported many problems that prevented them to read or focus on the texts. The UI in this task was semi-transparent, which can create strong conflicting depth cues between the text box and the video [66]. The difficulty is reinforced by the task characteristic. To read the whole sentence, participants need to focus longer on the UI, so they are more likely to see depth conflicts. In contrast, in the Search task participants did not experience depth conflicts, even though the three target scenes in this task contain large regions of close video objects. The Search task is characterized by rapid attention switching between the video and the UI, so participants might spent less time focusing on UI elements. Furthermore, the UI is opaque, so depth conflicts only occur around the edges and corners of the UI. As a results, the visual conflicts might have occurred peripherally.

These results suggest that depth conflict problems might depend on the nature of the 
task and the UI design. Depth conflicts are problematic when the UI is semi-transparent and when the task highly demands the viewer's attention. Depth conflicts are not as strong when the UI is opaque, or when the task can be done with peripheral vision.

Halo Blur did not work as well as expected. In the Subtitles task, even though the blur effects made the text box less transparent, we found that participants were still affected by conflicting depth cues from the video. This result is particularly interesting because blur has been known to reduce binocular rivalry [49] or weaken disparity depth cues [34]. However, most of these studies were done on static images. The text box used in our study follows the user's head movements. Thus, one potential explanation is that the users might have perceived motion cues from the video even in the blur regions. These cues could amplified the spatial information from the video images [92] and make blur less effective. We report the negative results of Halo Blur for the sake of completeness. The design and outcomes of this technique could also be interesting for future work in this area.

Dynamic Depth is a promising solution to depth conflicts. The ratings from the Subtitles task show that participants who used it reported the least problems. In the Search task, participants in both Baseline and Halo Blur conditions were slightly slower than in Dynamic Depth. We suspect that Dynamic Depth helped participants switch focus between the video and the UI faster because the UI was at the same depth of the video. Switching focus between large distances in depth often takes time [3]. However, since the current task duration is quite short, and we did not measure precise eye fixations, we could not confirm this observation.

Dynamic Depth detects depth conflicts in a conservative way by aggregating video disparity values around the UI. On one hand, it works well for small UI elements that are meant to be used together with video viewing. On the other hand, it may not work as well with more complex UI designs such as those that cover a large area of the video. The UI may overlay multiple video objects with varying depths. Integrating eye tracking to obtain more accurate video depth cues and developing solutions for large UI designs is an interesting 
direction and is left for future work.

Finally, we consider a few limitations of the user study. First, we did not measure participants' comprehension level in the Subtitles task. Methods to measure comprehension typically require participants to perform quizzes, which may add unnecessary stress to an already overwhelming VR experience (most of our participants had little or no experience with VR). Second, participants performed only short tasks in the study. Thus, we could not examine long-term effects of depth conflicts in VR video interfaces. This is an important research direction and should be investigated more in future. Third, the number of participants is rather low, which could limit the generalizability of our findings because of individual differences. Nevertheless, our study is a first step in exploring this problem and our results suggest a few promising insights that could be helpful for both application designers and researchers.

\subsection{Conclusion}

We explore depth conflicts between UI and stereoscopic video and discuss how they can affect user experience in VR video interfaces. We present two techniques to address this problem. Dynamic Depth detects and reduces depth conflicts by analyzing the video content and adjusting the depth of UI widgets to the depth of the video. Halo Blur simply blurs the video around the UI. We evaluated these techniques in a preliminary user study with two video tasks: watching video with subtitles and video searching. Our study compares our techniques with a baseline condition where the UI is fixed at a comfortable distance in VR. Our results suggest that the severity of depth conflict problems might depend on the task characteristics and the UI design. It also shows that Dynamic Depth is a promising solution and was most preferred by our participants for video subtitles. Our results also show that Halo Blur did not work as expected in a dynamic VR video environment. 


\section{Conclusion}

This dissertation introduced video workflow and systems that enable filmmakers to create and improve VR video while fully immersed in a VR headset. To conclude, this chapter will summarize the main contributions and discuss directions for future research.

\subsection{Summary of Contributions}

Broadly, this dissertation contributes new knowledge about the design requirements of video authoring workflows in VR and the development of new systems that enable these workflows.

\section{- Video editing in VR}

- A novel video editing workflow that enables filmmakers to edit VR video while fully immersed in a VR headset.

- Methods and user interfaces for accomplishing essential editing tasks in VR, including video navigation, assembling cuts, video bookmarks, and titling.

\section{- Collaborative video reviewing in VR}

- A novel video reviewing workflow that enables multiple filmmakers to collaborate and review VR video in VR.

- Networked video collaboration methods and user interfaces to enable faceto-face reviewing interactions in VR, including awareness visualization, view sharing, and notetaking. 


\section{- Depth conflict reduction techniques in VR}

- An analysis of how depth conflict problems can arise when user interface elements are rendered on top of stereoscopic VR video.

- Rendering techniques that adjust the appearance of the user interface based on the video content to reduce depth conflicts. One technique adjusts the perceived depth of the user interface to match that of the video content. Another technique simply blurs the video around the user interface.

\subsection{Future Directions}

\subsubsection{Interactivity in VR video}

Interactivity is a key aspect that makes VR video immersive. Today's VR technologies only enable viewers to look around in a $360^{\circ}$ environment, yet filmmakers have already begun exploring novel ways to tell stories with this interaction. However, beyond head rotation, we do not yet have a good understanding of other types of interaction that can enhance the experience of VR video. We need to investigate the design principles of interactive VR video and the authoring requirements for enabling them. As a motivating example, consider the potential of a VR video that can respond to user's view direction. The video author could mark important scene points in the video, such that the video could change orientation dynamically after a cut to help viewers follow the narrative without getting lost. The author could also make the video loop and wait for viewers to reach a pivotal scene, or "jumps" to different parts of the timeline to engage viewers in a non-linear branching story.

\subsubsection{VR video as a collaboration space}

VR technologies provide effective means to support collaboration, but most work has only focused on collaboration in 3D environments [9]. We believe that VR video can also serve 
as a collaborative space where multiple users can cooperate to create, study, and share immersive experiences. Within the film production domain, we can extend CollaVR [83] to support collaborative editing, which can enable multiple editors to brainstorm and try editing ideas together in the headset. Enabling group collaboration and the ability to try alternatives are two important considerations when designing creativity support tool [101]. Some key technical challenges will be in designing visualizations that allow editors to see each others' edits in real-time and ensuring that concurrent edits can be merged without conflicts.

We can also push the boundaries of collaborative VR video by broadening the application domains. In education, VR video can be used as a low-cost educational space to engage students in immersive experiences such as virtual field trips. New video collaboration and interaction techniques can be developed to make viewing video more similar to visiting a physical place. For example, consider a museum tour captured in VR video. The teacher can "lead" a group of students through different rooms, the students can form groups, share discussion, or inspect artifacts individually, all while wearing the VR headsets. In supporting remote collaboration, VR video can potentially enhance video conferencing. Thanks to recent advances in network technologies, video conferencing can be used to support realtime remote collaboration. Some examples are giving directions to tourists and guiding novices during home maintenance tasks [53]. With VR video, each viewer can have her own view, so instructions are not hindered by current problems that plague video conferencing such as bad viewpoints or unstable camera motion.

\subsubsection{Data-driven VR video authoring}

This dissertation introduced initial explorations in how individuals or small groups can author VR video experiences. One interesting question is what new insights we can learn from data collected from viewers of VR video. Watching and sharing VR videos on online websites 
like YouTube and Facebook have recently attracted considerable interests. Viewership data collected from VR viewers can potentially hint at novel cognitive insights such as presence, spatial awareness, memory, narrative engagement, and discomfort [73]. These insights are unique to VR video and have not been explored in existing data-driven video analysis techniques. One application is in modeling user's interest and comfort level in VR. Viewer models could then be integrated into authoring tools to help filmmakers create videos that are engaging and comfortable to watch. Another interesting direction is to investigate novel video playback mechanisms. When a person watches in VR, the video player can monitor her interest and compare to existing viewership data. In this way, the player can potentially adapt the video content to make the viewing experience more engaging. For examples, the cut could be automatically rotated to prevent disorientation or the pacing could be made slower when the user is confused. 


\section{Bibliography}

[1] Binocular vision, stereoscopic imaging and depth cues, 2017. Retrieved August 22, 2017 from https://developer.oculus.com/design/latest/concepts/bp_ app_imaging/

[2] Robert Anderson, David Gallup, Jonathan T. Barron, Janne Kontkanen, Noah Snavely, Carlos Hernández, Sameer Agarwal, and Steven M. Seitz. Jump: virtual reality video. ACM Transactions on Graphics, 35(6):1-13, nov 2016.

[3] Stephen R Arnott and Judith M Shedden. Attention switching in depth using randomdot autostereograms: Attention gradient asymmetries. Attention, Perception, \& Psychophysics, 62(7):1459-1473, 2000.

[4] Ruth Aylett and Sandy Louchart. Towards a narrative theory of virtual reality. Virtual Reality, 7(1):2-9, 2003.

[5] Ellen Baker, John Geirland, Tom Fisher, and Annmarie Chandler. Media Production: Towards Creative Collaboration Using Communication Networks. Computer Supported Cooperative Work (CSCW), 8(4):303-332, dec 1999.

[6] Paulo Bala, Mara Dionisio, Valentina Nisi, and Nuno Nunes. IVRUX: A tool for analyzing immersive narratives in virtual reality. In Ulrike Spierling and Nicolas Szilas, editors, Interactive Storytelling, volume 5334 of Lecture Notes in Computer Science, pages 3-11. Springer Berlin Heidelberg, 2016. 
[7] Jessica J. Baldis. Effects of spatial audio on memory, comprehension, and preference during desktop conferences. In Proceedings of the SIGCHI conference on Human factors in computing systems, number 3, pages 166-173, 2001.

[8] Joseph Bates. Virtual Reality, Art, and Entertainment. Presence: Teleoperators and Virtual Environments, 1(1):133-138, jan 1992.

[9] Steve Benford, Chris Greenhalgh, Tom Rodden, and James Pycock. Collaborative virtual environments. Communications of the ACM, 44(7):79-85, July 2001.

[10] Floraine Berthouzoz, Wilmot Li, and Maneesh Agrawala. Tools for placing cuts and transitions in interview video. ACM Trans. Graph., 31(4):67-1, 2012.

[11] Anastasia Bezerianos, Pierre Dragicevic, and Ravin Balakrishnan. Mnemonic Rendering: An Image-Based Approach for Exposing Hidden Changes in Dynamic Displays. In Proceedings of the 19th annual ACM symposium on User interface software and technology - UIST '06, page 159, New York, New York, USA, 2006. ACM Press.

[12] Tobias Blum, Matthias Wieczorek, Andre Aichert, Radhika Tibrewal, and Nassir Navab. The effect of out-of-focus blur on visual discomfort when using stereo displays. In 2010 IEEE International Symposium on Mixed and Augmented Reality, pages 13-17. IEEE, oct 2010.

[13] Natalia Bogdan, Tovi Grossman, and George Fitzmaurice. HybridSpace: Integrating 3D freehand input and stereo viewing into traditional desktop applications. In 2014 IEEE Symposium on 3D User Interfaces (3DUI), pages 51-58. IEEE, mar 2014.

[14] Wutthigrai Boonsuk, Stephen Gilbert, and Jonathan Kelly. The impact of three interfaces for 360-degree video on spatial cognition. In Proceedings of the SIGCHI Conference on Human Factors in Computing Systems - CHI '12, page 2579, New York, New York, USA, 2012. ACM Press. 
[15] Doug A. Bowman and Larry F. Hodges. An evaluation of techniques for grabbing and manipulating remote objects in immersive virtual environments. In Proceedings of the 1997 Symposium on Interactive 3D Graphics, I3D '97, pages 35-ff., New York, NY, USA, 1997. ACM.

[16] Jessica Brillhart. In the blink of a mind-attention, 2016. https://medium.com/thelanguage-of-vr/in-the-blink-of-a-mind-attention-1fdff60fa045.

[17] Jessica Brillhart. In the blink of a mind-prologue, 2016. https://medium.com/thelanguage-of-vr/in-the-blink-of-a-mind-prologue-7864c0474a29.

[18] David K. Broberg. Infrastructures for Home Delivery, Interfacing, Captioning, and Viewing of 3-D Content. Proceedings of the IEEE, 99(4):684-693, apr 2011.

[19] M. L. Brown, S. L. Newsome, and E. P. Glinert. An experiment into the use of auditory cues to reduce visual workload. In Proceedings of the SIGCHI conference on Human factors in computing systems, pages 339-346, 1989.

[20] Gerd Bruder, Frank Steinicke, Phil Wieland, and Markus Lappe. Tuning self-motion perception in virtual reality with visual illusions. IEEE Transactions on Visualization and Computer Graphics, 18(7):1068-1078, July 2012.

[21] Juan Casares, A Chris Long, Brad A Myers, Rishi Bhatnagar, Scott M Stevens, Laura Dabbish, Dan Yocum, and Albert Corbett. Simplifying video editing using metadata. In Proceedings of the 4th conference on Designing interactive systems: processes, practices, methods, and techniques, pages 157-166. ACM, 2002.

[22] Kai-Yin Cheng, Sheng-Jie Luo, Bing-Yu Chen, and Hao-Hua Chu. SmartPlayer: User-Centric Video Fast-Forwarding. In Proceedings of the SIGCHI Conference on Human Factors in Computing Systems - CHI '09, page 789, New York, New York, USA, apr 2009. ACM Press. 
[23] Mauro Cherubini, Marc-Antoine Nüssli, and Pierre Dillenbourg. Deixis and gaze in collaborative work at a distance (over a shared map): a computational model to detect misunderstandings. In Proceedings of the Symposium on Eye Tracking Research \& Applications, pages 173-180, 2008.

[24] Pei-Yu Chi, Joyce Liu, Jason Linder, Mira Dontcheva, Wilmot Li, and Bjoern Hartmann. Democut: generating concise instructional videos for physical demonstrations. In Proceedings of the 26th annual ACM symposium on User interface software and technology, pages 141-150. ACM, 2013.

[25] Jacqueline Chu, Chris Bryan, Min Shih, Leonardo Ferrer, and Kwan-Liu Ma. Navigable Videos for Presenting Scientific Data on Affordable Head-Mounted Displays. In Proceedings of the 8th ACM on Multimedia Systems Conference - MMSys'17, number VIDi, pages 250-260, New York, New York, USA, 2017. ACM Press.

[26] cineSync, 2017. https://cospective.com/cinesync/.

[27] Andy Cockburn, Amy Karlson, and Benjamin B Bederson. A review of overview+detail, zooming, and focus+context interfaces. ACM Computing Surveys, 41(1):1-31, dec 2008.

[28] Bleeding Disorders Community. Factor treatment in hemophilia a and b. Video, June 2017. Retrieved August 22, 2017 from https://wWw . youtube . com/watch? $\mathrm{v}=\mathrm{tHbFY5G3GVs}$

[29] Maxime Cordeil, Tim Dwyer, Karsten Klein, Bireswar Laha, Kim Marriott, and Bruce H. Thomas. Immersive collaborative analysis of network connectivity: CAVEstyle or head-mounted display? IEEE Transactions on Visualization and Computer Graphics, 23(1):441-450, January 2017. 
[30] Joao L C Dasilva. period film scene full vr 360 3d on film set. Video, September 2017. Retrieved August 22, 2017 from https://www .youtube.com/watch?v= nH1xJox61z4.

[31] Nicholas Davis, Alexander Zook, Brian O’Neill, Brandon Headrick, Mark Riedl, Ashton Grosz, and Michael Nitsche. Creativity support for novice digital filmmaking. In Proceedings of the SIGCHI Conference on Human Factors in Computing Systems CHI'13, page 651, New York, New York, USA, 2013. ACM Press.

[32] IRALTA VR Productora de video. 360 3d the circus school carampa. Video, February 2016. Retrieved August 22, 2017 from https://www .youtube.com/watch?v= tABJ1VwW-2M.

[33] devinsupertramp. Terrifying masquerade party in 3d 360!! Video, February 2017. Retrieved August 22, 2017 from https://www .youtube.com/watch?v=mb_J_ bMor1g.

[34] Piotr Didyk, Tobias Ritschel, Elmar Eisemann, Karol Myszkowski, Hans-Peter Seidel, and Wojciech Matusik. A luminance-contrast-aware disparity model and applications. ACM Transactions on Graphics, 31(6):1, nov 2012.

[35] Paul Dourish and Victoria Bellotti. Awareness and coordination in shared workspaces. In Proceedings of the ACM conference on Computer-supported cooperative work, pages 107-114, November 1992.

[36] Adeola Fabola, Alan Miller, and Richard Fawcett. Exploring the past with google cardboard. In Digital Heritage, 2015, volume 1, pages 277-284. IEEE, 2015.

[37] Kevin Fan, Liwei Chan, Daiya Kato, Kouta Minamizawa, and Masahiko Inami. VR Planet: Interface for Meta-View and Feet Interaction of VR Contents. In $A C M$ 
SIGGRAPH 2016 VR Village on - SIGGRAPH'16, pages 1-2, New York, New York, USA, 2016. ACM Press.

[38] Steven Feiner, Blair MacIntyre, Marcus Haupt, and Eliot Solomon. Windows on the world: $2 \mathrm{~d}$ windows for $3 \mathrm{~d}$ augmented reality. In Proceedings of the 6th annual ACM symposium on User interface software and technology, pages 145-155. ACM, 1993.

[39] Ajoy S. Fernandes and Steven K. Feiner. Combating VR sickness through subtle dynamic field-of-view modification. In Proceedings of the IEEE Symposium on 3D User Interfaces, pages 201-210, March 2016.

[40] Mike Fraser, Steve Benford, Jon Hindmarsh, and Christian Heath. Supporting awareness and interaction through collaborative virtual interfaces. In Proceedings of the ACM symposium on User interface software and technology, volume 1, pages 27-36, 1999.

[41] Dustin E.R. Freeman, Stephanie Santosa, Fanny Chevalier, Ravin Balakrishnan, and Karan Singh. LACES: Live Authoring through Compositing and Editing of Streaming Video. In Proceedings of the SIGCHI Conference on Human Factors in Computing Systems - CHI '14, pages 1207-1216, New York, New York, USA, 2014. ACM Press.

[42] Davide Gadia, Marco Granato, Dario Maggiorini, Laura Anna Ripamonti, and Cinzia Vismara. Consumer-oriented Head Mounted Displays: Analysis and Evaluation of Stereoscopic Characteristics and User Preferences. Mobile Networks and Applications, (February):1-11, feb 2017.

[43] ZDF Enterprises GmbH. Gladiators in the roman colosseum vr 3d 360. Video, February 2017. Retrieved August 22, 2017 from https://wWW.youtube.com/ watch?v=XBuijx_iZtQ. 
[44] Carl Gutwin and Saul Greenberg. Design for individuals, design for groups: tradeoffs between power and workspace awareness. In Proceedings of the ACM conference on Computer supported cooperative work, pages 207-216.

[45] Jefferson Han and Brian Smith. CU-SeeMe VR immersive desktop teleconferencing. In Proceedings of the ACM international conference on Multimedia, pages 199-207, 1996.

[46] Jeffrey Heer and Maneesh Agrawala. Design considerations for collaborative visual analytics. Information Visualization, 7(1):49-62, January 2008.

[47] Rorik Henrikson, Bruno De Araujo, Fanny Chevalier, Karan Singh, and Ravin Balakrishnan. Multi-device storyboards for cinematic narratives in VR. Proceedings of the ACM Symposium on User interface software and technology, pages 787-796, 2016.

[48] Rorik Henrikson, Bruno De Araujo, Fanny Chevalier, Karan Singh, and Ravin Balakrishnan. Storeoboard: sketching stereoscopic storyboards. In Proceedings of the SIGCHI conference on Human factors in computing systems, pages 4587-4598, 2016.

[49] David M Hoffman and Martin S Banks. Focus information is used to interpret binocular images. Journal of vision, 10(5):13, 2010.

[50] Hong Hua. Enabling Focus Cues in Head-Mounted Displays. Proceedings of the IEEE, 105(5):805-824, may 2017.

[51] Jingwei Huang, Zhili Chen, Duygu Ceylan, and Hailin Jin. 6-DOF VR videos with a single 360-camera. In 2017 IEEE Virtual Reality (VR), pages 37-44. IEEE, 2017.

[52] Edwin L. Hutchins, James D. Hollan, and Donald A. Norman. Direct Manipulation Interfaces. Human-Computer Interaction, 1(4):311-338, dec 1985. 
[53] Brennan Jones, Anna Witcraft, Scott Bateman, Carman Neustaedter, and Anthony Tang. Mechanics of Camera Work in Mobile Video Collaboration. In Proceedings of the 33rd Annual ACM Conference on Human Factors in Computing Systems - CHI '15, pages 957-966, New York, New York, USA, 2015. ACM Press.

[54] Shunichi Kasahara and Jun Rekimoto. JackIn Head: Immersive Visual Telepresence System with Omnidirectional Wearable Camera for Remote Collaboration. In Proceedings of the 21st ACM Symposium on Virtual Reality Software and Technology VRST'15, pages 217-225, New York, New York, USA, 2015. ACM Press.

[55] Robert S Kennedy, Norman E Lane, Kevin S Berbaum, and Michael G Lilienthal. Simulator sickness questionnaire: An enhanced method for quantifying simulator sickness. The international journal of aviation psychology, 3(3):203-220, 1993.

[56] Don Kimber, Jonathan Foote, and Surapong Lertsithichai. FlyAbout: Spatially Indexed Panoramic Video. In Proceedings of the ninth ACM international conference on Multimedia - MULTIMEDIA '01, page 339, New York, New York, USA, 2001. ACM Press.

[57] Eugenia M. Kolasinski. Simulator sickness in virtual environments. Technical Report ARI-TR-1027, Army Research Institute for the Behavioral and Social Sciences, 1995.

[58] Johannes Kopf. 360 video stabilization. ACM Transactions on Graphics (TOG), 35(6):195, 2016.

[59] Gregory Kramida and Amitabh Varshney. Resolving the Vergence-Accommodation Conflict in Head Mounted Displays. IEEE Transactions on Visualization and Computer Graphics, 22(7):1-16, 2015.

[60] Matthew W Kreuter, Melanie C Green, Joseph N Cappella, Michael D Slater, Meg E Wise, Doug Storey, Eddie M Clark, Daniel J OâĂŹKeefe, Deborah O Erwin, Kath- 
leen Holmes, et al. Narrative communication in cancer prevention and control: a framework to guide research and application. Annals of behavioral medicine, 33(3):221-235, 2007.

[61] Arun Kulshreshth and Joseph J. LaViola. Dynamic Stereoscopic 3D Parameter Adjustment for Enhanced Depth Discrimination. In Proceedings of the 2016 CHI Conference on Human Factors in Computing Systems - CHI'16, pages 177-187, New York, New York, USA, 2016. ACM Press.

[62] Oh-Hyun Kwon, Chris Muelder, Kyungwon Lee, and Kwan-Liu Ma. A Study of Layout, Rendering, and Interaction Methods for Immersive Graph Visualization. IEEE Transactions on Visualization and Computer Graphics, 22(7):1802-1815, 2016.

[63] Jeremy Lacoche, Morgan Le Chenechal, Sebastien Chalme, Jerome Royan, Thierry Duval, Valerie Gouranton, Eric Maisel, and Bruno Arnaldi. Dealing with frame cancellation for stereoscopic displays in 3D user interfaces. In 2015 IEEE Symposium on 3D User Interfaces (3DUI), pages 73-80. IEEE, mar 2015.

[64] M. Lambooij, M.J. Murdoch, W.A. IJsselsteijn, and I. Heynderickx. The impact of video characteristics and subtitles on visual comfort of 3D TV. Displays, 34(1):8-16, jan 2013.

[65] Marc T. M. Lambooij, Wijnand A. IJsselsteijn, and Ingrid Heynderickx. Visual discomfort in stereoscopic displays: a review. 6490(May 2010):64900I, 2007.

[66] Robert S. Laramee and Colin Ware. Rivalry and interference with a head-mounted display. ACM Transactions on Computer-Human Interaction, 9(3):238-251, sep 2002. 
[67] Mackenzie Leake, Abe Davis, Anh Truong, and Maneesh Agrawala. Computational video editing for dialogue-driven scenes. ACM Transactions on Graphics, 36(4):1-14, jul 2017.

[68] Hao Li, Laura Trutoiu, Kyle Olszewski, Lingyu Wei, Tristan Trutna, Pei-Lun Hsieh, Aaron Nicholls, and Chongyang Ma. Facial performance sensing head-mounted display. ACM Transactions on Graphics (Proceedings of SIGGRAPH), 34(4), July 2015.

[69] LookAt.io, 2017. https://www.lookat.io/.

[70] Thomas Löwe, Michael Stengel, Förster Emmy-Charlotte, Steve Grogorick, and Marcus Magnor. Visualization and analysis of head movement and gaze data for immersive video in head-mounted displays. Proceedigns of the Workshop on Eye Tracking and Visualization, 1:1-5, 2015.

[71] Zheng Lu and Kristen Grauman. Story-driven summarization for egocentric video. In Proceedings of the IEEE Conference on Computer Vision and Pattern Recognition, pages 2714-2721, 2013.

[72] Bruce D. Lucas and Takeo Kanade. An iterative image registration technique with an application to stereo vision. In Proceedings of the 7th International Joint Conference on Artificial Intelligence - Volume 2, IJCAI'81, pages 674-679, San Francisco, CA, USA, 1981. Morgan Kaufmann Publishers Inc.

[73] Andrew MacQuarrie and Anthony Steed. Cinematic virtual reality: Evaluating the effect of display type on the viewing experience for panoramic video. In Virtual Reality (VR), 2017 IEEE, pages 45-54. IEEE, 2017.

[74] Charles Malleson, Maggie Kosek, Martin Klaudiny, Ivan Huerta, Jean-Charles Bazin, Alexander Sorkine-Hornung, Mark Mine, and Kenny Mitchell. Rapid one-shot 
acquisition of dynamic VR avatars. In Proceedings of IEEE VR, pages 131-140, 2017.

[75] John Mateer. Directing for Cinematic Virtual Reality: how the traditional film director's craft applies to immersive environments and notions of presence. Journal of Media Practice, 18(1):14-25, jan 2017.

[76] Kevin Matzen, Michael F. Cohen, Bryce Evans, Johannes Kopf, and Richard Szeliski. Low-cost 360 stereo photography and video capture. ACM Trans. Graphics, 2017.

[77] Mark McGill, John H Williamson, and Stephen Brewster. Examining the role of smart TVs and VR HMDs in synchronous at-a-distance media consumption. ACM Transactions on Computer-Human Interaction, 23(5):1-57, November 2016.

[78] Britta Meixner. Hypervideos and Interactive Multimedia Presentations. ACM Computing Surveys, 50(1):1-34, mar 2017.

[79] Ming Hou. A model of real-virtual object interactions in stereoscopic augmented reality environments. In Proceedings on Seventh International Conference on Information Visualization, 2003. IV 2003., volume 2003-Janua, pages 512-517. IEEE Comput. Soc, 2003.

[80] Meredith Ringel Morris and Eric Horvitz. SearchTogether: an interface for collaborative web search. In Proceedings of the ACM symposium on User interface software and technology, page 3, 2007.

[81] Alessandro Mulloni, Hartmut Seichter, Andreas Dünser, Patrick Baudisch, and Dieter Schmalstieg. $360^{\circ}$ panoramic overviews for location-based services. In Proceedings of the SIGCHI Conference on Human Factors in Computing Systems - CHI '12, page 2565, New York, New York, USA, 2012. ACM Press. 
[82] Luís a. R. Neng and Teresa Chambel. Get around $360^{\circ}$ hypervideo. In Proceedings of the 14th International Academic MindTrek Conference on Envisioning Future Media Environments - MindTrek'10, page 119, New York, New York, USA, 2010. ACM Press.

[83] Cuong Nguyen, Stephen DiVerdi, Aaron Hertzmann, and Feng Liu. CollaVR: Collaborative in-headset review for VR video. In Proceedings of the 30th Annual Symposium on User Interface Software and Technology - UIST '17. ACM, 2017.

[84] Cuong Nguyen, Stephen DiVerdi, Aaron Hertzmann, and Feng Liu. Vremiere: InHeadset Virtual Reality Video Editing. In Proceedings of the 2017 CHI Conference on Human Factors in Computing Systems - CHI'17, pages 5428-5438, New York, New York, USA, 2017. ACM Press.

[85] Thomas Oskam, Alexander Hornung, Huw Bowles, Kenny Mitchell, and Markus Gross. OSCAM - optimized stereoscopic camera control for interactive 3D. In Proceedings of the 2011 SIGGRAPH Asia Conference on - SA '11, page 1, New York, New York, USA, 2011. ACM Press.

[86] Costas Panagiotakis and George Tziritas. A speech/music discriminator based on RMS and zero-crossings. IEEE Transactions on Multimedia, 7(1):155-166, February 2005.

[87] Amy Pavel, Dan B Goldman, Björn Hartmann, and Maneesh Agrawala. VidCrit: video-based asynchronous video review. In Proceedings of the Symposium on User interface software and technology, pages 517-528, 2016.

[88] Javier Sánchez Pérez, Enric Meinhardt-Llopis, and Gabriele Facciolo. Tv-11 optical flow estimation. Image Processing On Line, 2013:137-150, 2013. 
[89] Benjamin Petry and Jochen Huber. Towards effective interaction with omnidirectional videos using immersive virtual reality headsets. In Proceedings of the 6th Augmented Human International Conference on - AH'15, pages 217-218, New York, New York, USA, 2015. ACM Press.

[90] Julien Phalip, Ernest a. Edmonds, and David Jean. Supporting remote creative collaboration in film scoring. In Proceeding of the ACM conference on Creativity and cognition, page 211, 2009.

[91] Suporn Pongnumkul, Jue Wang, and Michael Cohen. Creating map-based storyboards for browsing tour videos. In Proceedings of the 21 st annual ACM symposium on User interface software and technology - UIST'08, pages 13-22, New York, NY, USA, 2008. ACM.

[92] Stephan Reichelt, Ralf Häussler, Gerald Fütterer, and Norbert Leister. Depth cues in human visual perception and their realization in $3 \mathrm{~d}$ displays. In Proc. SPIE, volume 7690, page 76900B, 2010.

[93] Road to VR. Facebook social VR demo. 2016. https://www.youtube.com/watch?v=YuIgyKLPt3s.

[94] Gustavo Alberto Rovelo Ruiz, Davy Vanacken, Kris Luyten, Francisco Abad, and Emilio Camahort. Multi-viewer gesture-based interaction for omni-directional video. In Proceedings of the SIGCHI Conference on Human Factors in Computing Systems CHI'14, pages 4077-4086, New York, New York, USA, 2014. ACM Press.

[95] Leila Schemali and Elmar Eisemann. Design and evaluation of mouse cursors in a stereoscopic desktop environment. In 2014 IEEE Symposium on 3D User Interfaces (3DUI), pages 67-70. IEEE, mar 2014. 
[96] Jonas Schild, Liane Bölicke, Joseph J. LaViola Jr., and Maic Masuch. Creating and analyzing stereoscopic 3D graphical user interfaces in digital games. In Proceedings of the SIGCHI Conference on Human Factors in Computing Systems - CHI '13, page 169, New York, New York, USA, 2013. ACM Press.

[97] Jonas Schild, Joseph J. LaViola, and Maic Masuch. Altering gameplay behavior using stereoscopic 3D vision-based video game design. In Proceedings of the 32nd annual ACM conference on Human factors in computing systems - CHI '14, pages 207-216, New York, New York, USA, 2014. ACM Press.

[98] Matthew Schnipper. The rise and fall and rise of virtual reality, 2014. http://www.theverge.com/a/virtual-reality.

[99] Klaus Schoeffmann, Marco A Hudelist, and Jochen Huber. Video Interaction Tools : A Survey of Recent Work. ACM Computing Surveys, 48(1):1-34, sep 2015.

[100] Anne Sèdes, Pierre Guillot, and Eliott Paris. The HOA library, review and prospects. In International Computer Music Conference I Sound and Music Computing, pages $855-860$, September 2014.

[101] Ben Shneiderman. Creativity support tools: accelerating discovery and innovation. Communications of the ACM, 50(12):20-32, dec 2007.

[102] A. Smolic, S. Poulakos, S. Heinzle, P. Greisen, M. Lang, A. Hornung, M. Farre, N. Stefanoski, O. Wang, L. Schnyder, R. Monroy, and M. Gross. Disparity-aware stereo 3D production tools. Proceedings - 2011 Conference for Visual Media Production, CVMP 2011, pages 165-173, 2011.

[103] Dave Snowdon, Elizabeth F Churchill, and Alan J Munro. Collaborative virtual environments: digital spaces and places for CSCW. Collaborative Virtual Environments, pages 1-34, 2000. 
[104] Filippo Speranza, Wa J Tam, Ron Renaud, and Namho Hur. Effect of disparity and motion on visual comfort of stereoscopic images. In Andrew J. Woods, Neil A. Dodgson, John O. Merritt, Mark T. Bolas, and Ian E. McDowall, editors, Proceedings of SPIE: Stereoscopic Displays and Virtual Reality Systems XIII, volume 6055, page 60550B, feb 2006.

[105] Yu-Chuan Su, Dinesh Jayaraman, and Kristen Grauman. Pano2vid: Automatic cinematography for watching $360^{\circ}$ videos. CoRR, abs/1612.02335, 2016.

[106] Ivan E Sutherland. A head-mounted three dimensional display. In Proceedings of the December 9-11, 1968, fall joint computer conference, part I, pages 757-764. ACM, 1968.

[107] Richard Szeliski and Heung-Yeung Shum. Creating full view panoramic image mosaics and environment maps. In Proceedings of the 24th annual conference on Computer graphics and interactive techniques, pages 251-258. ACM Press/AddisonWesley Publishing Co., 1997.

[108] Wa James Tam, Filippo Speranza, Carlos Vázquez, Ron Renaud, and Namho Hur. Visual comfort: stereoscopic objects moving in the horizontal and mid-sagittal planes. In Andrew J. Woods, Nicolas S. Holliman, and Gregg E. Favalora, editors, Proc. SPIE, volume 8288, page 828813, feb 2012.

[109] Anthony Tang and Omid Fakourfar. Watching $360^{\circ}$ videos together. In Proceedings of the SIGCHI conference on Human factors in computing systems, 2017.

[110] Anh Truong, Floraine Berthouzoz, Wilmot Li, and Maneesh Agrawala. Quickcut: An interactive tool for editing narrated video. In Proceedings of the 29th Annual Symposium on User Interface Software and Technology, UIST '16, pages 497-507, New York, NY, USA, 2016. ACM. 
[111] Michael Tsang, George W. Fitzmaurice, Gordon Kurtenbach, Azam Khan, and Bill Buxton. Boom chameleon: simultaneous capture of 3D viewpoint, voice and gesture annotations on a spatially-aware display. In Proceedings of the 15th annual ACM symposium on User interface software and technology - UIST '02, volume 4, page 111, New York, New York, USA, 2002. ACM Press.

[112] James R. Wallace, Stacey D. Scott, Eugene Lai, and Deon Jajalla. Investigating the role of a large, shared display in multi-display environments. Computer Supported Cooperative Work, 20(6):529-561, December 2011.

[113] Junle Wang, Patrick Le Callet, Sylvain Tourancheau, Vincent Ricordel, and Matthieu Perreira Da Silva. Study of depth bias of observers in free viewing of still stereoscopic synthetic stimuli. Journal of Eye Movement Research, 5(5), 2012.

[114] Colin Ware. Dynamic stereo displays. Proceedings of the SIGCHI conference on Human factors in computing systems - CHI '95, pages 310-316, 1995.

[115] Haijun Xia, Bruno Araujo, Tovi Grossman, and Daniel Wigdor. Object-Oriented Drawing. In Proceedings of the SIGCHI Conference on Human Factors in Computing Systems - CHI '16, pages 4610-4621, New York, New York, USA, 2016. ACM Press.

[116] Robert Xiao and Hrvoje Benko. Augmenting the Field-of-View of Head-Mounted Displays with Sparse Peripheral Displays. In Proceedings of the SIGCHI Conference on Human Factors in Computing Systems - CHI '16, pages 1221-1232, New York, New York, USA, 2016. ACM Press.

[117] Yong Jung, Hosik Sohn, Seong-il Lee, and Yong Ro. Visual comfort improvement in stereoscopic 3D displays using perceptually plausible assessment metric of visual comfort. IEEE Transactions on Consumer Electronics, 60(1):1-9, feb 2014. 
[118] Dongwook Yoon, Nicholas Chen, François Guimbretière, and Abigail Sellen. RichReview: blending ink, speech, and gesture to support collaborative document review. In Proceedings of the ACM symposium on User interface software and technology, pages 481-490, 2014. 